\title{
Calcitonin Activity of Particulate Fractions of the Ovine Thyroid Gland
}

Peter Rudolf Oeltgen

Loyola University Chicago

Follow this and additional works at: https://ecommons.luc.edu/luc_diss

\section{Recommended Citation}

Oeltgen, Peter Rudolf, "Calcitonin Activity of Particulate Fractions of the Ovine Thyroid Gland" (1973). Dissertations. 1315.

https://ecommons.luc.edu/luc_diss/1315

This Dissertation is brought to you for free and open access by the Theses and Dissertations at Loyola eCommons. It has been accepted for inclusion in Dissertations by an authorized administrator of Loyola eCommons. For more information, please contact ecommons@luc.edu. (c) $($ ) $\Theta \Theta$

This work is licensed under a Creative Commons Attribution-Noncommercial-No Derivative Works 3.0 License. Copyright (c) 1973 Peter Rudolf Oeltgen 


\title{
CAICITONIN ACTIVITY OF PARTICULATE FRACTIONS OF THE OVINE THYROID GLAND
}

$$
\text { by }
$$

Peter Rudolf Oeltgen

A Dissertation Submitted to the Faculty of the Graduate School of Loyola University in Partial Fulfillment of the Requirements for the Degree of Doctor of Philosophy

\author{
February
}

1973 


\section{LIFE}

Peter R. Oeltgen was born on January 29, 1942 in Ottendorf, Germany.

He graduated from Wheeler High School of North Stonington, Connecticut, in 1960. In June, 1965, he received a Bachelor of Science degree from DePaul University, Chicago, Illinois. Graduate studies at DePaul University were begun in September, 1967 and he was awarded a Master of Science degree in Biological Sciences in June, 1969, following completion of his thesis on "Characterization of alkaline phosphatase in amphibian tissues and the influence of temperature and parathyroid hormone on enzyme activity."

In July, 1969, Peter Oeltgen began his graduate studies in the Department of Biochemistry and Biophysics at Loyola University, Stritch School of Medicine, under the guidance and counsel of Dr. Maurice V. L'Heureux. From July, 1969 to June, 1972, he served as a graduate teaching assistant in the department. He was a Trainee in a National Institute of Health Training Grant in Biochemistry from July, 1969 to July, 1970.

Peter Oeltgen is the co-author of the following papers: McWhinnie, D. J., P. R. Oeltgen, and D. Stiles. (1971) Amphibian Alkaline Phosphatase - I. Biochemical characterization of 

alkaline phosphatase in tissues of Rana pipiens. Comp. Biochem. Physiology 38B, 247-262.

McWhinnie, D. J. and P. R. Oeltgen. (1971) Amphibian Alkaline Phosphatase - II. The influence of parathyroid extract on alkaline phosphatase in tissues of Rana pipiens. Comp. Biochem. Physiol. 38B, 739-751. 


\section{ACKNOWLEDGEMENTS}

The author is indebted to Dr. Maurice V. L'Heureux for his continued guidance, support, and encouragement extending from the beginning of this study to its completion. His integrity as a teacher, advisor, and a citizen have made an indelible impression upon the author.

My sincere gratitude is also extended to Dr. Robert Schleuter and Mr. Richard Weeks of Armour Pharmaceutical Company, Kankakee, Illinois for providing the standard calcitonin preparation utilized in this investigation and for the photography of polyacrylamide gels.

The author is also very grateful to $\mathrm{Dr}$. John U. Balis of the Department of Pathology, Loyola University, Stritch School of Medicine, for the electronmicrographs depicted in this dissertation.

Finally, the author wishes to express his sincerest gratitude to his wife, Jean, for her extremely competent typing of the dissertation, for her constructive criticism, scientific and other, and for her forbearance and unselfish support during this investigation. 
CHAPTER

Calcitonin

Historical Development . . . . . . .

Biological Assay and Radioimmunoassay . .

Chemistry . . . . . . . . . . . .

Sites and Mode of Action . . . . . . .

Mechanism of Action . . . . . . . .

Control of Secretion ............

Metabolism and Duration of Action . . . .

Role in Clinical Disorders of Calcium

Metabolism ...............

Physiological Role... . . . . . . 46

Statement of the Problem . . . . . . . 50

General Experimental Procedure . . . . . 53

Preparation of Incubation Media . . . . . 56

Tissue Preparation and Incubation . . . . 58

Isólation of Subcellular Fractions . . . . . 59

Determination of Serum Calcium . . . . . 69

Protein Analysis . . . . . . . . . . . 71

Measurement of Radioactivity . . . . . . 75

Determination of Biological Activity . . . 76 


\section{TABLE OF CONTENTS CONTINUED}

Polyacrylamide Gel Electrophoresis of Microsomal and Standard Calcitonin Preparations . . . . . . . . . . . . .

Standard Procedure--Anionic Gel Solutions . . 82

Standard Procedure--Cationic Gel Solutions . . 94

Densitometry of Polyacrylamide Gels . . . . 98

Photography of Polyacrylamide Gels . . . . 99

Chemicals Required for Polyacrylamide Gel

Electrophoresis

Isoelectric Focusing . . . . . . . . 100

The Influence of Increasing the Dose of the Subcellular Fraction on Hypocalcemic Activity.

The Influence of Physiological and High Calcium Concentrations in the Incubation Medium on the Hypocalcemic Response of Subcellular Fractions Isolated from Incubated Thyroid Slices . . . . . . . .

The Influence of Varying Calcium Concentrations in the Incubation Medium on the Hypocalcemic Response of Subcellular Preparations Isolated from Incubated Thyroid Slices and on the Extent of Incorporation of Glycine-C-14 into these Fractions . . . .

Characterization of Microsomal and Standard Calcitonin Preparations by Polyacrylamide Gel Electrophoresis . . . . . . . . .

Characterization of Microsomal and Standard Calcitonin Preparations by Isoelectric Focusing . . . . . . . . . . . . . . . . 
CHAPTER

PAGE

IV

DISCUSSION . . . . . . . . . . . . .

169

V SUMMARY AND CONCLUSIONS . . . . . . . . . . 183

BIBLIOGRAPHY . . . . . . . . . . . . 186 


\section{LIST OF TABLES}

TABLE

PAGE

I Standard Curve Data for Protein Determination . .

II Anionic Gel Stock Solutions... . . . . . .

III Anionic Gel Working Solutions . . . . . . . .

IV Anionic Gel Fixative, Staining and Destaining

Solutions . . . . . . . . . . . . . . .

V Cationic Gel Stock Solutions . . . . . . . .

VI Cationic Gel Working Solutions . . . . . .

VII Hypocalcemic Activity of Increasing Doses of Microsomal Preparations Obtained from

Incubation Media Containing Physiological

Calcium Concentrations . . . . . . . . . . .

VIII Hypocalcemic Activity of Mitochondrial and Nuclear Preparations at a Concentration of 2,000 $\mu$ g Obtained from Incubation Media Containing Physiological Calcium Concentrations .

IX Hypocalcemic Activity of Microsomal, Mi tochondrial and Nuclear Preparations at a Concentration of 2,000 $\mathrm{\mu g}$ Obtained from Incubation Media Containing High Calcium Concentrations

$\mathrm{X}$ Hypocalcemic Activity of Heat-Treated Microsomal Preparation at a Concentration of 2,000 $\mathrm{ug}$ Obtained from Incubation Media Containing Physiological Calcium Concentrations.

XI Hypocalcemic Activity of Pepsin-Treated Microsomal Preparation at a Concentration of 2,000 $\mu \mathrm{g}$ Obtained from Incubation Media Containing Physiological Calcium Concentrations.

XII Hypocalcemic Activity of Microsomal, Mitochondrial, and Nuclear Preparations obtained from Incubation Media Containing 


\section{LIST OF TABLES CONTINUED}

TABLE

PAGE

High and Physiological Calcium

Concentrations . . . . . . . . . . . . .

XII Hypocalcemic Activity of Microsomal,

Mitochondrial, and Nuclear Preparations

Obtained from Incubation Media Containing

Physiological Calcium Concentrations and

$2.5 \mu \mathrm{Ci}$ of Glycine-C-14 (UL) . . . . . . . .

XIV Hypocalcemic Activity of Microsomal,

Mitochondrial, and Nuclear Preparations

obtained from Incubation Media Containing

Low Calcium Concentrations and $2.5 \mu \mathrm{Ci}$ of

Glycine-C-14 (UL)

XV Hypocalcemic Activity of Microsomal,

Mitochondrial, and Nuclear Preparations

obtained from Incubation Media Containing

High Calcium Concentrations and $2.5 \mu \mathrm{Ci}$ of

Glycine-C-14 (UL) . . . . . . . . . . .

XVI Hypocalcemic Activity of Purified

Microsomal, Mitochondrial, and Nuclear

Preparations obtained from Incubation Media

Containing Low, Physiological, and $\mathrm{High}$

Calcium Concentrations

XVII The Influence of Varying Calcium Concentrations on the Hypocalcemic Response of Microsomal

Preparations

XVIII Hypocalcemic Activity of Electrophoretically

Resolved Microsomal Preparations obtained

from an Incubation Medium Containing a

Physiological Calcium Concentration and $2.5 \mu \mathrm{Ci}$

of Glycine-C-14 (UL) .............

XIX The Hypocalcemic Response of Electrophoreticaliy

Resolved Microsomal Preparations 


\section{LIST OF FIGURES}

1 Amino Acid Sequence of Ovine Calcitonin ......

2 Amino Acid Sequence of Porcine, Bovine, Ovine, Human, and Salmon Calcitonin (2 pages)..

3 Fractionation Scheme Utilized to Obtain Mitochondrial, Nuclear, and Microsomal Fractions from Incubated ovine Thyroid Gland Slices (2 pages)

4 Electron Micrograph of the Thyroid Microsomal Fraction $(105,000 \times \mathrm{g})$ Containing Smooth and Rough Endoplasmic Reticulum, Free Ribosomes, and Dense Granules . . . . . . . . . . . . .

5 Electron Micrograph of a Crude Mitochondrial Fraction (7,000 x g) Exhibiting Some Microsomal Contamination . . . . . . . . . .

6 Phase Contrast Photograph of a Purified Thyroid Nuclear Fraction $(50,000 \times \mathrm{g})$. . . . .

7 Standard Curve for Protein Determination ....

8 Disc Electrophoresis Apparatus . . . . . . .

9 Schematic Representation of a Polyacrylamide Gel Electrophoresis... . . . . . . . .

10 The Polymerization Reaction of Polyacrylamide..

11 Schematic Representation of the Electrofocusing of Proteins

12 LKB 8100 Electrofocusing Column with Completed Separation of a Mixture of Heroglobins A, S, and $\mathrm{C}$ in a $\mathrm{pH}$ Gradient of 7 to 9 ........

13 Separation of Hemoglobin A, S, and C Mixture by Electrofocusing 
14 Protein Content, Radioactivity, and Elution Pattern of Lyophilized Microsomal Preparation from a Physiological Calcium Concentration Incubation Medium Passed through a Column of Sephadex G-100 . . . . . . . . . . . .

15 Protein Content, Radioactivity, and Elution Pattern of Lyophilized Mitochondrial Preparation from a Physiological Calcium Concentration Incubation Medium Passed through a Column of Sephadex G-100 . . . . . .

16 Protein Content, Radioactivity, and Elution Pattern of Lyophilized Nuclear Preparation from a Physiological Calcium Concentration Incubation Medium Passed through a Column of Sephadex G-100 . . . . . . . . . . . . . .

17 Protein Content, Radioactivity, and Elution Pattern of Lyophilized Microsomal Preparation from a Low Calcium Concentration Incubation Medium Passed through a Column of Sephadex G-100.

18 Protein Content, Radioactivity, and Elution Pattern of Lyophilized Mitochondrial Preparation from a Low Calcium Concentration Incubation Medium Passed through a Column of Sephadex G-100 . . . . . . . . . . .

19 Protein Content, Radioactivity, and Elution Pattern of Lyophilized Nuclear Preparation from a Low Calcium Concentration Incubation Medium Passed through a Column of Sephadex G-100.

20 Protein Content, Radioactivity, and Elution Pattern of Lyophilized Microsomal Preparation from a High Calcium Concentration Incubation Medium Passed through a Column of Sephadex G-100.

21 Protein Content, Radioactivity, and Elution Pattern of Lyophilized Mitochondrial Preparation from a High Calcium Concentration 
Incubation Medium Passed through a Column of Sephadex G-100 . . . . . . . . . . . .

22 Protein Content, Radioactivity, and Elution Pattern of Lyophilized Nuclear Preparation from a High Calcium Concentration Incubation Medium Passed through a Column of Sephadex G-100. . . . . . . . . . . . . . . .

23 Densitometer Trace of a Standard Calcitonin Preparation Resolved in a Cationic Polyacrylamide Gel System . . . . . . . . . . .

24 Densitometer Traces of Electrophoretically

Resolved Hormone Preparations in Anionic

Polyacrylamide Gels . . . . . . . . . . . . .

25 Polyacrylamide Gels Representing Electrophoretically Resolved Standard and Microsomal Calcitonin Preparations

26 Separation of $7 \mathrm{mg}$ of a Standard Calcitonin Preparation Having a Potency of 88 MRC Units by the LKB 8100 Electrofocusing Column in a $\mathrm{pH}$ Gradient Extending from $\mathrm{pH} 3$ to 6 . . . . . .

27 Separation of $14 \mathrm{mg}$ of Sephadex G-50 Purified Microsomal Preparation by the LKB 8100 Electrofocusing Column in a pH Gradient Extending from 3 to 5 . . . . . . . 
CHAPTER I

INTRODUCTION

\section{CALCITONIN}

\section{HISTORICAL DEVELOPMENT}

Calcium is among the best regulated ionic constituents of extracellular fluid in mammals. Many vital processes, including muscular contraction, neuromuscular excitability, membrane permeability, blood clot formation, and the activity of a number of enzyme systems are critically dependent on the level of ionic calcium. At blood calcium levels below 4 to $5 \mathrm{mg} / 100 \mathrm{ml}$, some mammalian forms enter into tetanic convulsions, often resulting in death, while blood calcium levels of 12 to $13 \mathrm{mg} / 100 \mathrm{ml}$ in most mammals often results in cardiac disturbances and renal calcification which may also prove fatal. Therefore, the precise regulation of blood calcium concentration within a narrow range is of vital importance in maintaining mineral hoemostasis and hence normal physiological and metabolic function of all mammalian forms.

The regulation of blood calcium level requires an internal. balance between calcium mobilization from the skeleton and calcium deposition in new and maturing bone. The latter process depends on the rate of new bone deposition and on the concentrations of ionic calcium and phosphate in blood. Diminished levels of either calcium or phosphate will interfere with this process resulting in rickets 
or

osteomalacia and defective mineral deposition on bone matrix. The precise regulation of plasma calcium, especially in young manmals, is dependent on the metabolic interaction of two hormones, parathyroid hormone (PTH) and calcitonin (CT), both of which influence calcium mobilization from bone, by as yet, undetermined mechanisms.

The first calcium-regulating hormone to be discovered was the parathyroid hormone which is secreted by the parathyroid glands. In 1880, Sandstrom, a Swedish anatomist, presented a detailed description of these minute endocrine glands which have an intimate association with the thyroid glands of many mammalian species. However, he was unaware of their physiological role in maintaining mineral homeostasis of mammals. Gley (1891) was the first investigator to recognize the physiological importance of the parathyroid glands, since their unintentional removal during total thyroidectomy in dogs often resulted in tetanic convulsions and ultimate death of the animals. However, their important role in calcium metabolism was determined by MacCallum and Voegtlin (1909), who demonstrated that parathyroidectomy resulted in a precipitous fall in plasma calcium, associated with tetany and convulsions. These symptoms could be alleviated by calcium administration. Collip (1925) successfully prepared an acid extract of parathyroid tissue which proved to be biologically potent since it elevated plasma calcium levels in normal dogs and alleviated the hypocalcemia and tetany in parathyroidectomized dogs.

Rasmussen and Craig (1959) and Aurbach (1959), working independently, announced almost simultaneously, the isolation of essentially 
pure PTH by phenol extraction of bovine parathyroid glands and countercurrent distribution of the extracted material. Rasmussen and Craig, utilizing ultracentrifugation and dialysis, estimated that the molecular weight of their purified extract was on the order of $7,000 \pm 1,500$.

Niall et al. (1970) determined the complete amino acid sequence of the bovine PTH molecule, consisting of $84^{\circ}$ amino acids and having a molecular weight of 9,500. Studies by Potts et al. (1968) have established that the amino-terminal 30 residues of the 84 amino acid molecule are an absolute requisite for full biological activity. This has recently been verified by Potts et al.(1971) who have synthesized biologically active fragments of bovine PTH comprising the 34 amino acid sequence from the amino terminus of the molecule. The synthetic 34 amino acid peptide exhibited all of the specific properties of the native molecule which included stimulation of adenyl cyclase in both bone and kidney cells, elevation of blood calcium in rats and dogs, and increased excretion of cyclic AMP and phosphate in rats.

In contrast to the slow progression of parathyroid hormone research, encompassing a 63 year period since MacCallum and Voegtlin (1909) elucidated the role of the parathyroid glands in calcium metabolism, the progression of calcitonin research has been extremely rapid since the proposal of Copp et al. (1961) of a hormone involved in regulating the "tone" of calcium in body fluids. The series of experiments which led to the identification, isolation, structural analysis, synthesis and characterization of the physiological role of calcitonin have all been successfully accomplished within the last 11 years. 
Prior to the discovery of calcitonin, the precision of calcium homeostasis, the maintainence of blood calcium concentration in a narrow range, was thought to depend entirely on the parathyroid glands and the interaction of PTH with the calcium reservoir in the skeleton. McLean and Urist (1955) and McLean (1957) had proposed a simple negative feedback system in which a fall in plasma calcium prompted the parathyroid glands to secrete additional amounts of PMH, which consequently increased bone resorption and the release of calcium from the skeleton. McLean (1957) proposed that there was no positive means by which the organism could respond to hypercalcemia, the only control being that of parathyroid hormone secretion. The first definitive study which clearly demonstrated the inadequacy of this concept was performed by Sanderson et al. (1960). These investigators demonstrated that in thyroparathyroidectomized dogs there was an inability to adequately respond to either an acute calcium load or calcium deprivation. The hypocalcemia which resulted could readily be attributed to removal of the parathyroid glands; however, the impaired recovery of these animals from the effects of calcium infusions could not be readily explained by the prevailing concept of calcium regulation. Sanderson et al. (1960) did not realize the import of these findings. It remained for Copp and coworkers to evaluate the effects on systemic blood calcium of acute stimulation or suppression of parathyroid glandular activity. In a Series of classic experiments, Copp and Davidson (1961) and Copp et al. (1961, 2962) evaluated parathyroid gland activity by noting the effects of acute calciun load or calcium deprivation on blood calcium levels. Regional perfusion of the dog thyroid-parathyroid 
gland complex was performed by successive 2 hour perfusion periods with blood containing higher $(+2 \mathrm{mg} / 100 \mathrm{ml})$ or lower $(-2 \mathrm{mg} / 100 \mathrm{ml})$ calcium concentrations than normal levels. Hypocalcemic perfusion of the gland complex resulted in hypercalcemia through the action of increased PTH secretion, an anticipated result; however, the hypocalcemia which developed more rapidly after hypercalcemic perfusion of the isolated thyroid-parathyroid gland complex as compared to the hypocalcemia which resulted after total thyroparathyroidectomy could not be accounted for by simple suppression of PTH secretion. Therefore, Copp and coworkers proposed the existence of a humoral factor which exerted a positive control on hypercalcemia. Copp and Henze (1964) proposed that the source of this hypocalcemic factor was the parathyroid glands, and that it be called calcitonin because it was exerting a positive role in regulating the level or "tone" of calcium in plasma and extracellular fluids.

Copp and coworkers" proposal of the existence of a hypocalcemic hormone which played a significant role in calcium homeostasis proved to be correct; however, their proposal that calcitonin originated from the parathyroid gland proved to be incorrect.

Hirsch, Gauthier, and Munson (1963) made a second and highly significant observation. These investigators noted that parathyroidectomy by the Erdheim cautery technique, which involves hot wire cauterization of adjacent thyroid tissue in the rat, resulted in a much more rapid and profound hypocalcemia than simple surgical parathyroidectomy. Thus, the postulation by Hirsch et al. (1963), that the cautery procedure released into the circulation a potent hypocalcemic substance stored in the thyroid glands, proved to be 
correct. Hirsch et al. (1964) were able to demonstrate that injection of as little as $0.1 \mathrm{mg}$ of an acidic extract of rat thyroid gland into recipient intact rats prompted a marked hypocalcemia. Hirsch and coworkers assigned the name, thyrocalcitonin, to their thyroid extract in order to distinguish the gland of origin, although their extract was possibly identical to the factor studied by copp and his associates. Since their isolation of a hypocalcemic hormone from the thyroid glands, this hormone has been referred to by both names, calcitonin and thyrocalcitonin. However, the name, calcitonin, is gaining more acceptance since potent hypocalcemic extracts have recently been isolated from non-thyroid sources such as the ultimobranchial glands of both mammalian and non-mammalian forms thereby ruling out the thyroid gland as the exclusive source for this hormone.

The first definitive experiments confirming the ability of the thyroid gland to protect against hypercalcemia and ruling out the parathyroid gland as an important factor were supplied by Talmage et al. (1964, 1965). These investigators used rats with functional parathyroid gland transplants, thereby allowing them to determine the effects of acute calcium load in thyroidectomized as well as parathyroidectomized animals. Talmage et al. (1965) determined that rats with intact thyroids could respond adequately to calcium load while thyroidectomized animals could not. Moreover, parathyroidectomy did not significantly alter this response.

Foster et al. (1964) demonstrated that hypercalcemic perfusion of isolated external parathyroid glands of the goat which had all thyroid tissue removed did not result in any hypocalcemic effect. 
However, hypercalcemic perfusion of the thyroid lobe with its contained internal parathyroid resulted in a $15 \%$ decrease in systemic plasma calcium indicating that the thyroid was essential for a hypocalcemic effect. Confirmatory evidence for the release of a hypocalcemic factor from the thyroid glands in response to hypercalcemic perfusion was presented by Care (1965). Care examined serial sections of perfused pig thyroid tissue and demonstrated that it contained no parathyroid tissue which could affect the response to hypercalcemic perfusion.

\section{CELL OF ORIGIN}

With the unequivocal demonstration by Care (1965) that the thyroid gland was the gland of origin of calcitonin, it was then possible to investigate the cell of origin of this hypocalcemic hormone. Munson et al. (1966) presented evidence which ruled out the possibility that calcitonin was synthesized and released from the thyroid follicular cells. These investigators demonstrated that thyroid-stimulating hormone (TSH), essential for thyroxine and triiodothyronine production of the follicular cells, did not alter the calcitonin content of the thyroid glands.

Attention was therefore focused on the "light". cell or parafollicular cell, a cell type of the thyroid which previously had no function assigned to it. This cell type had been referred to as the

"light" cell by stux et al. (1961) because it stained poorly witr periodic acid-Schiff reagent. These cells were examined by Nonidez in 1931. He described the cells in the thyroid glands of dogs as 
arising in the follicular epithelium, later migrating into the interfollicular spaces, and remaining around the periphery of the follicle; hence, the term "parafollicular" cells. Nonidez noted the presence of argyrophile granules within these cells and proposed that "the granules represented the antecedent of an endocrine secretion poured directly into the vessels."

Wissig (1962) presented the first report of the electron microscopy of the parafollicular cells of the rat thyroid. He indicated that these cells had a high content of spherical vesicles which were contained within their cytoplasm. These subcellular structures measured between 1500 and $2000 \&$ in diameter and some contained electron-dense material which appeared as discrete granules. Pearse (1966) demonstrated that these cells were primarily epifollicular (oriented towards blood vessels rather than the follicular lumen) in pigs while in man these cells appear to be both epifollicular and parafollicular. He therefore referred to these cells as "C" cells to indicate their possible role in calcitonin secretion. The name, "C" cell, avoided reference to the staining qualities of the cells and to their precise localization. For this reason, the new name, "C" cell, could be applied to all species and to organs and tissues other than the thyroid.

There are three lines of experimental evidence that, combined, conclusively identify the "C" cells as the source of calcitonin. First, these cells were sensitive to small changes in serum calcium levels. Foster et al. (1964) had demonstrated that the "C" cells responded to high calcium concentrations by discharging their electron dense vesicular content. Similarly, Matsuzawa and Kurosumi 
(1967) demonstrated that during hypercalcemic stimulation of the rat with calcium chloride, there was a decrease in secretory granule number reaching a minimum at 4 hours after injection. Secondly, an immunofluorescent-antibody technique developed by Bussolati and Pearse (1967) based on antiserum obtained from guinea pigs injected with highly purified porcine CT, indicated that CT in the pig thyroid was present in cells which were morphologically indistinguishable from the "C" cells. Thirdly, a preferential uptake of 5-hydroxytryptophan (5-HTP) by mouse thyroid "C" cells was described by Ritzen et al. (1965) while Larsen et al. (1966) noted that the biogenic amine precursor, L-dihydroxyphenylalanine (1-DOPA) was stored selectively by these cells. By utilizing specific staining techniques, these compounds would exhibit their characteristic fluorescence in the "C" cells. Pearse and Carvalheiro (1967) took advantage of this unexplained amine concentrating mechanism of the "C" cells to determine the embryological origin of these cells. Since both 1-DOPA and 5-HTP cross the placenta freely, these compounds were injected into pregnant mice and rats and the initial appearance of fluorescent "C" cells in the embryos was monitored. Pearse and Carvalheiro (1967) first noticed fluorescence on the 12 th day in the fourth pharyngeal pouch and the ultimobranchial body of 14-day old rat embryos. The fluorescent "C" cells, comprising the ultimobranchial body, finally fused with the thyroid gland and assumed their characteristic distribution within the thyroid.

Final procf of the ultimobranchial origin of CT was obtained by Copp, Cockcroft, and Kueh, in studies on the hypocalcemic effect of extracts of these glands in chickens and turkeys (Copp, Cockcroft, 
and Kueh, 1967a), and in dogfish shark (Copp, Cockroft, and Kueh, 1967b), in amphibia (bullfrog) and reptiles (Copp and Parkes, 1968), and in fish (salmon) (O'Dor et al., 1969). In addition, Copp and coworkers demonstrated that CT was absent from the thyroids of birds which had potent ultimobranchial CT. In all of these species, the ultimobranchial gland was separate and distinct from the thyroid. Subsequent work with the isolation and characterization of the biologically active material from the ultimobranchial glands of salmon (0'Dor et al., 1969b), has established that the ultimobranchial glands contain CT that has many similarities to mammalian CT. Moreover, the salmon hormone exhibited 25 times the biological activity of the most potent mammalian extracts.

\section{BIOLOGICAL ASSAY AND RADIOIMMUNOASSAY}

\section{Biological Assay}

Determination of the amino acid sequence and chemical synthesis of CT necessitated the development of purification procedures leading to the isolation of the hormone in pure form. Hence, it was essential that a reliable biological assay for CT be developed in order to assess extent of purification at each stage of the isolation procedure. The rat and the mouse proved to be ideal test animals for assaying the hypocalcemic activity of CT preparations (Hirsch et al., 1964; Kumar et al., 1965). Most researchers today utilize the rat assay of Hirsch et al. (1964), or a modification of this method. The assay of Hirsch et al. (1964) was 
based on the decline in plasma calcium 1 or 1.5 hours after intravenous or subcutaneous injection of rat thyroid extracts into 100 to 150 gram rats.

In 1966, the Division of Biological Standards of the National Institute of Medical Research, Mill Hill, London, England, established an international reference standard which was termed "Thyroid Calcitonin Research Standard A." This was a crude but stable acid extract of porcine thyroid glands prepared in accordance with the salt fractionation procedure of Baghdiantz et al. (1964).

The Medical Research Council unit (MRC Unit) was defined as the calcium lowering activity contained in four ampoules ( $40 \mathrm{mg}$ or $10 \mathrm{mg} /$ ampoule) which was equivalent to $250 \mu \mathrm{g}$ protein/mg and 0.25 MRC units per ampoule. Approximately 0.01 MRC unit of this material given intravenously in the biological assay procedure of Kumar et al. (1965), in which blood calcium is determined 50 minutes after injection of test solution in rats, produced a 10 percent fall in plasma calcium. In 1967, Research Standard A was superseded by Research Standard $B$, a more highly purified, but less stable preparation of porcine CT furnished by Dr. Robert Schleuter of Armour Pharmaceutical Company, Kankakee, Illinois to the Nedical Research Council. This preparation was standardized against Research Standard A by international collaborative assays (Parsons and Reynolds, 1968) that established the ratio of specific biological activity of $A$ to $B$ to be equal to 1 to 900 . Standard $B$ had a potency of $22 \mathrm{MRC}$ units/mg. 
Radioimmunoassay

Deftos et al. (1968) and Lee et al. (1969) developed a highly sensitive and specific radioimmunoassay for porcine CT whereby as little as 15 to $20 \mu \mu g$ of porcine hormone could be detected reproducibly. Their studies with rabbits indicated that CT circulated in the blood of the normal animal, that secretion of the hormone increased 15-fold in response to induced hypercalcemia ( $\mathrm{CaCl}_{2}$ load), and that the disappearance of CT in the blood was rapid. The halflife of secreted CT was estimated to be 5 to 15 minutes. Deftos and coworkers were also able to demonstrate CT in normal and malignant human thyroid tissue. This meant that normal human $\mathrm{CT}$, which could not be detected by biological assay except in patients with thyroid carcinoma, could now be detected routinely by the highly sensitive radioimmunoassay procedure.

\section{CHEMISTRY}

Hirsch et al. (1963) prepared the first potent extracts of calcitonin from rat thyroid glands, The injection of extract from as little as one-third of a rat thyroid gland resulted in a marked hypocalcemia in recipient normal and thyroidectomized rats one hour after injection. However, Hirsch and coworkers selected hog rather than rat thyroid extract for purification procedures due to the availability and abundance of material, even though the biological activity of hos CT preparations was less than rat CT extracts. Also, contamination with parathyroid tissue was not of concern since the parathyroid glands of the rog are embedded at sites remote from the 
thyroid gland and in the thymus gland. Hirsch et al. (1964) accomplished a ten-fold purification of the starting acid extracts of hog thyroids by centrifuging this material at $100,000 \times \mathrm{g}$ for 24 hours. The supernatant was hypocalcemic in intact rats. A subsequent two-step purification procedure consisting of fractionation on Sephadex G-50, and Sephadex G-25 resulted in a total 500-fold purification of calcitonin from the starting extract.

An effective alternative method for partial purification of porcine CT, utilizing dried, defatted thyroid tissue which subse-quently underwent salt fractionation (2-3 $\mathrm{M} \mathrm{NaCl})$ and gel filtration on Sephadex G-100 columns was reported by Baghdiantz et al. (1964).

Baghdiantz et al. (1964), Hirsch et al. (1964), and Tashjian and Warnock (1967) also demonstrated the peptide nature and the concomitant loss of biological activity of their relatively impure CT preparations. These hypocalcemic preparations were readily inactivated by incubating them with a variety of proteolytic enzymes such as trypsin and pepsin.

Further purification of porcine material was accomplished by Tenenhouse et al. (1965) who successfully applied a method for the partial purification of bovine PTH. Hog thyroid glands were dehydrated and defatted by successive extractions with acetone and chloroform. The resulting powder was treated with $8 \mathrm{M}$ urea $-0.2 \mathrm{~N}$ $\mathrm{HCI}-0.1 \mathrm{M}$ cysteine at $4^{\circ} \mathrm{C}$ for one hour, followed by solvent and salt fractionation, precipitation of the active material with trichloroacetic acid (TCA), and recovery of this precipitate as a lyophilized powder. This TCA powder was passed through a column of 
Sephadex G-75 and yielded a preparation which Tenenhouse et al. (1965) considered to be homogeneous after fractionation by starch gel electrophoresis. This hypocalcemic preparation was given the name, TAR peptide, since Tenenhouse, Arnaud, and Rassmussen (1965) devised the isolation procedure for its preparation. These investigators determined the molecular weight of the TAR peptide, which they presumed to be pure CT, to be 8700 on the basis of amino acid composition. Munson et al. (1966 and 1968) repeated the work of Tenenhouse et al. (1965) and demonstrated that more retarded eluates from Sephadex columns yielded CT preparations having up to 10 times the biological activity of the TAR peptide. Munson and coworkers clearly demonstrated that the TAR peptide could be further fractionated into 5 bands on highly sensitive analytical polyacrylamide gels thereby furnishing conclusive evidence that the TAR peptide was still a heterogeneous preparation. Potts et al. (1967) and Munson et al. (1968) utilized preparative polyacrylamide gel electrophoresis to obtain a porcine CT preparation which represented a 50,000fold purification of the starting acetone-chloroform dried extract. Potts et al. (1967) determined the molecular weight of their most purified porcine CT extract to be 4,000 as calculated from their estimate of the amino acid composition. This value was in rather close agreement with the value of 5,000 to 6,000 as determined by the sucrose density centrifugation procedure of $0^{\prime}$ Riordan et al. (1966) and the Sephadex G-100 gel filtration experiments of McIntyre et al. (1965) in which a molecular weiglt of less than 3,000 was assigned to their CT preparations. The first correct report of the amino acid composition of porcine CT was presented by Putter et al. (1967). 
Based on the findings of McIntyre et al. (1965), O'Riordan et al. (1966) and Potts et al. (1967), all of whom had extracted and isolated CT by different techniques, the molecular weight of the hormone was variously estimated at 3,000 to 6,000. Final proof of isolation of essentially pure CT necessitated the determination of the entire amino acid sequence, chemical synthesis of a peptide with the proposed structure, and demonstration that the synthetic peptide had the hypocalcemic activity of the isolated, naturally occurring CT. All of these extremely important projects were successfully accomplished by a number of research groups in 1968.

The complete amino acid sequence of pure porcine calcitonin was reported by Potts et al. (1968). It was determined through the use of sequential degradation by the phenylisothiocyanate procedure, digestion with exopeptidases, and isolation and analysis of peptide subfragments resulting from cleavage of the polypeptide by trypsin, pepsin, chymotrypsin, papain, cyanogen bromide, and limited hydrolysis in dilute acid and concentrated acid. Neher et al. (1968) simultaneously arrived at the identical amino acid sequence for porcine CT and Bell et al. (1968) arrived at the same sequence finding the weight-average molecular weight of porcine $C T$ to be 3700 , which was in excellent agreement with the value of 3604 as required by the amino acid sequence of the molecule. Distinctive structural features of the 32 amino acid molecule are that the proportion of charged amino acids is low, the carboxyl-terminal amino acid is prolinamide, and there is a 1-? intrachain disulfide bridge, providing a 23-membered ring at the amino terminus. Brewer et al. (1968) demonstrated that pure porcine CT did not contain more than $0.02 \%$ 
iodine, indicating the distinct difference between this molecule and the iodinated thyroid hormones. Rictel et al. (1968) and Guttmann et al. (1968) confirmed the sequence determination of porcine CT by chemical synthesis of a polypeptide which possessed the same specific biological activity as the isolated, naturally occurring hormone.

Utilizing gel filtration and ion-exchange chromatographic methods, Brewer et al. (1970) isolated bovine CT and determined its amino acid sequence while Potts et al. (1970), utilizing similar techniques, determined the amino acid composition of ovine CT. Sauer et al. (1970) succeeded in determining the amino acid sequence of ovine CT which is depicted in Figure 1. The specific biological activity of the bovine and ovine calcitonin when compared to the porcine molecule was found to be closely similar, ranging from 50120 Medical Research Council Units/mg. The amino acid composition of these three calcitonin differed by only a few residues.

Neher et al. (1968) isolated and determined the complete amino acid sequence of human CT from extracts of a single medullary carcinoma of the thyroid. This tumor exhibited an overabundance of "C" cells when compared to normal human thyroid tissue. The human CT molecule also consisted of 32 amino acids; however, 18 of the 32 amino acid residues differed from the corresponding positions occupied in the porcine CT molecule. Riniker et al. (1968) presented evidence that human tumor CT existed in two forms. The first, termed CT "M", consisted of 32 amino acids, while the seccnd, CT "D", proved to be an antiparallel dimer of CT "N". The dimer could be converted into the monomer by treatment with base. Riniker 


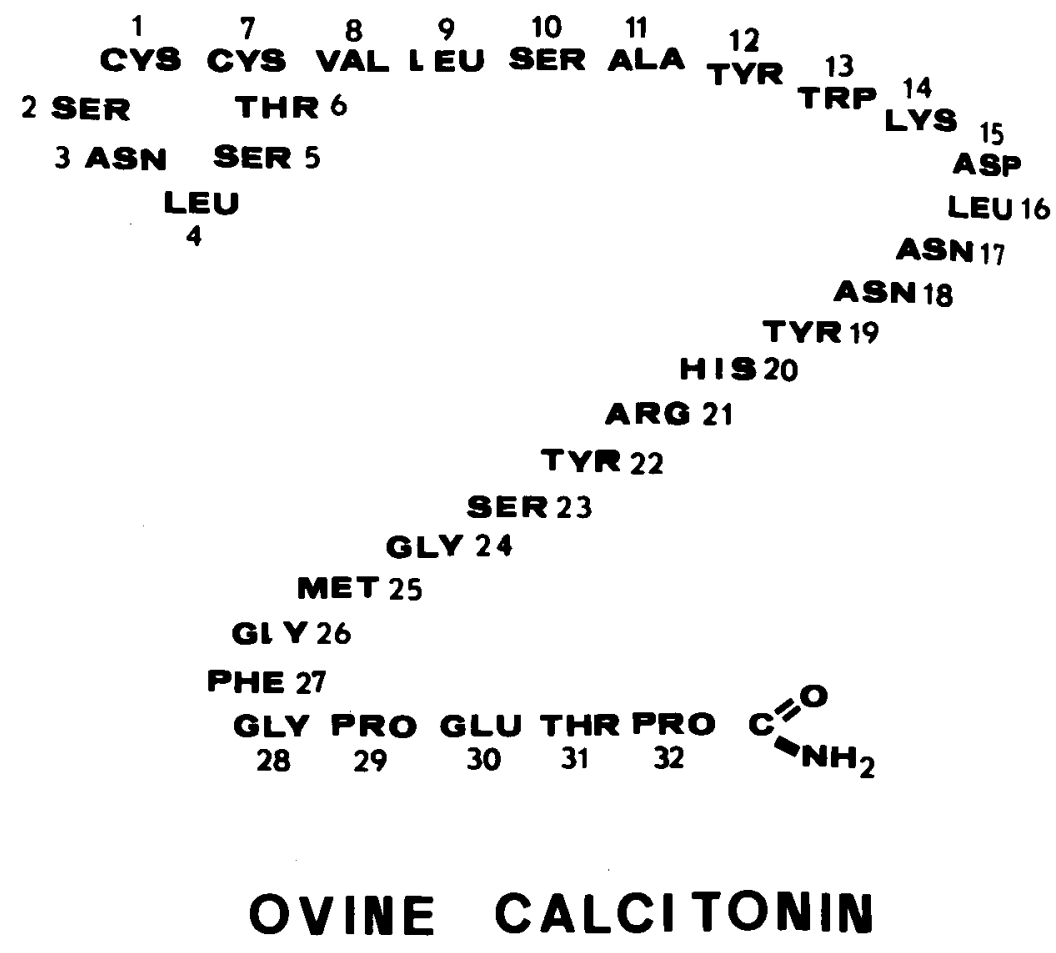

Figure 1. The amino acid sequence of ovine calcitonin (Sauer et al. 1970). 
and coworkers proposed that the dimer exhibited full biological activity. However, no dimer of porcine, bovine, or ovine CT has been identified. The concept of a biologically active dimer of human CT was revised when McIntyre (1970) presented evidence which suggested the dimer was without activity until its gradual conversion into an active monomer by incubation for one hour with bovine plasma albumin.

Copp et al. (1967) had demonstrated the ultimobranchial origin of CT in several submammalian vertebrate species and he and his associates ( $0^{\prime}$ Dor et al., 1969) successfully isolated CT in homogeneous form from salmon. These extracts were extremely potent in mammalian bioassay systems. Niall et al. (1969) achieved final purification of salmon ultimobranchial CT in quantities sufficient to permit sequential determination of the molecule by use of the Edman degradation method. The salmon molecule possesses, in common with other calcitonins, a 32-amino acid peptide chain terminating in prolinamide and a 1-? intrachain disulfide bridge. Keutmann et al. (1970) detected two chemically different forms of salmon CT termed CT I and II. The major isohormonal form, CT I, had the 32 amino acid sequence determined by Niall et al. (1969), while the minor component, CT II, exhibited similar biological potency to the major peptide having a specific activity in rabbit of 2400 MRC units per mg as compared to 2700 MRC units for the major component, or 10 times the potency of the most active mammalian preparations.

The amino acid sequence of CT molecules from 5 different species, (porcine, bovine, ovine, human, and salmon), is now known and is depicted in Figure 2. Each of the five calcitonin molecules con- 


\section{PORCINE CALCITONIN}

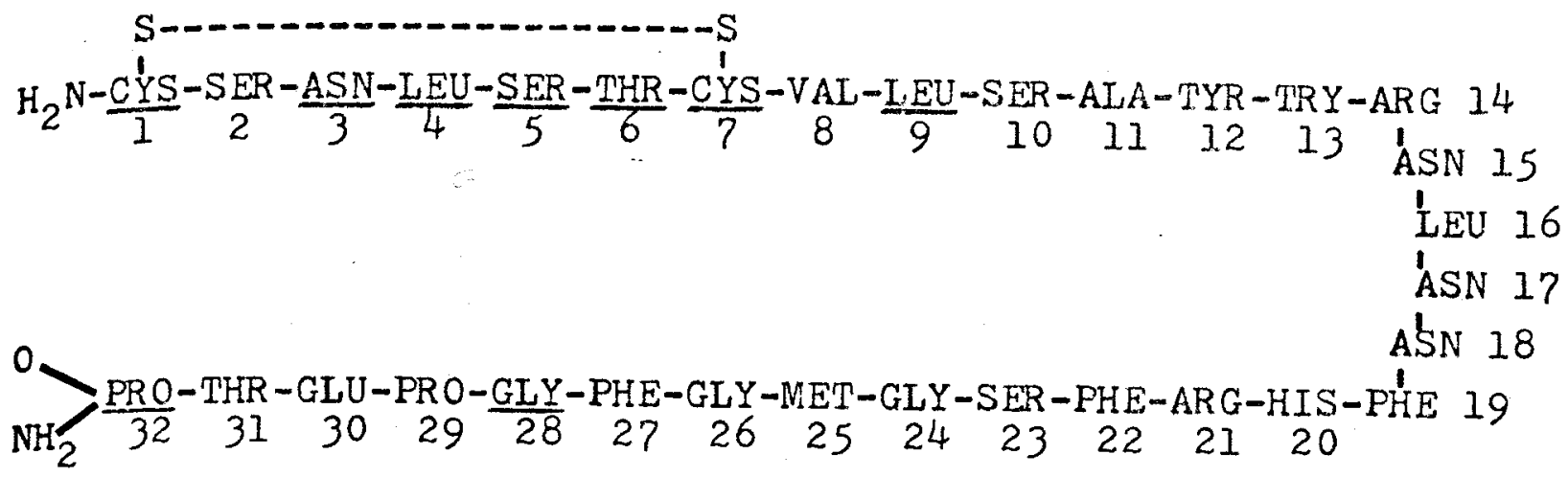

BOVINE CALCITONIN

$\mathrm{H}_{2} \mathrm{~N}-\mathrm{CY} S-\mathrm{S} E R-$ ASN-IEU-SER-THR-CY'S-VAL-LEU-SER-ALA-TYR-TRY-LYS 14 ÁsP 15 LEU 16 ÁSN 17

NH $\frac{\text { PRO-THR-GLU-PRO-GLY-PHE-GLY-MET-GLY-SER-PHE-ARG-HIS-TYR } 19}{32}{ }_{31}$

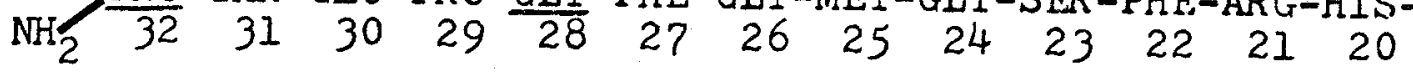

\section{OVINE CALCITONIN}

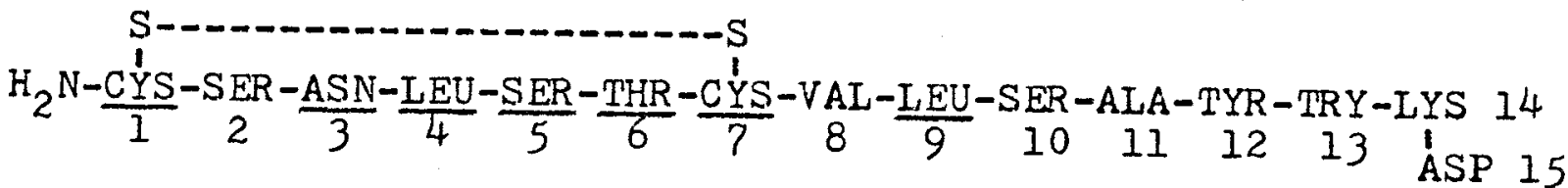
LEU 16 ASN 17

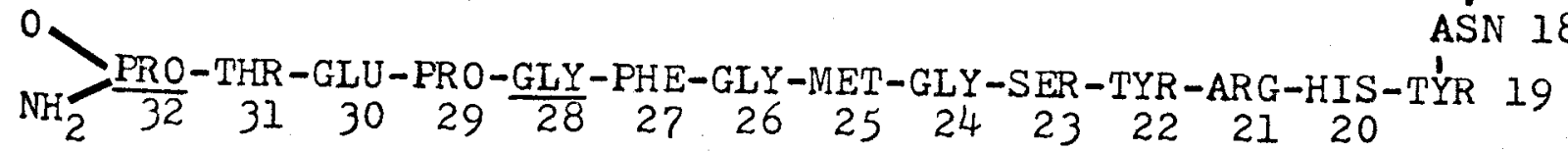

Figure 2. The complete amino acid sequence of porcine (Potts et al. 1968), bovine (Brewer et al. 1970), ovine (Sauer et al. 1970), human (Neher et al. 1968), and salmon (Niall et al. 1969) calcitonin. 
Figure 2, continued

\section{HUMAN CALCITONIN}

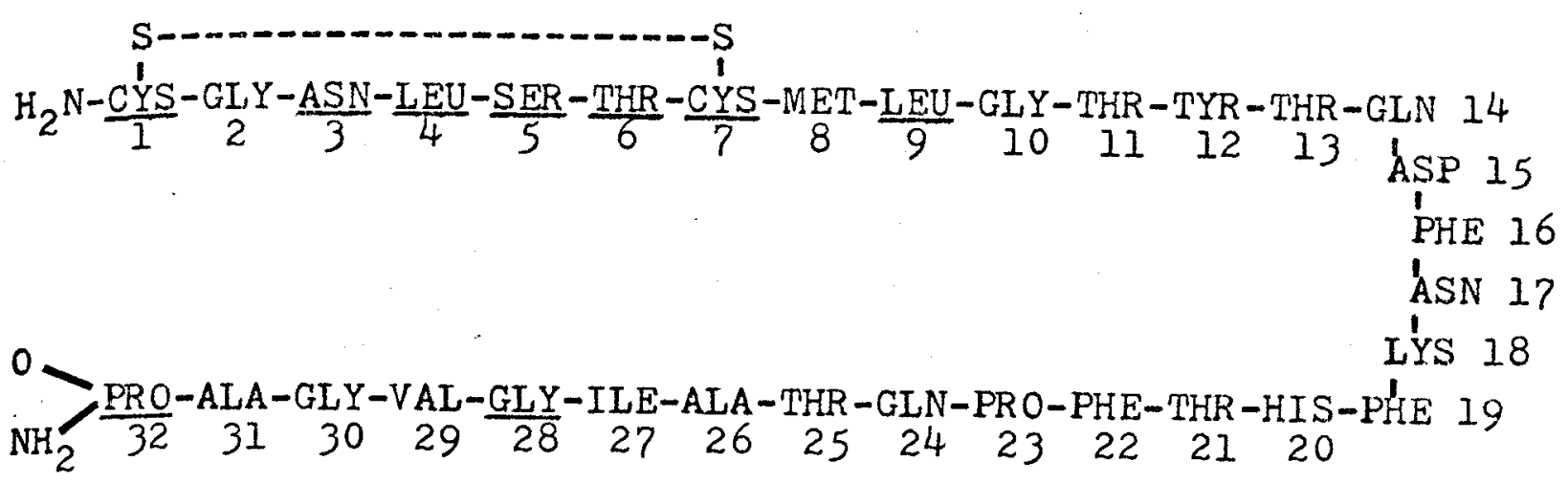

\section{SALMON CALCITONIN}

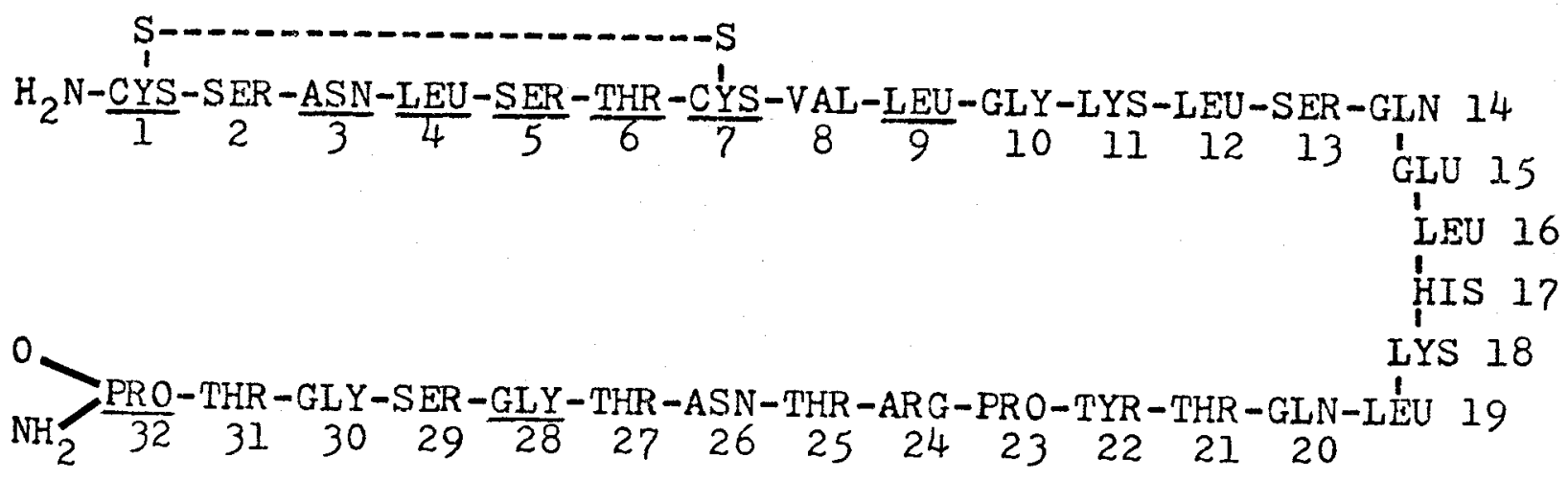


sists of a 32 amino acid chain exhibiting a 1-7 amino terminal disulfice bridge and a carboxyl terminal prolinamide. Only nine amino acids occupy identical sequence positions in the five calcitonin molecules depicted in Figure 2. Seven of these nine occur in the amino terminal disulfide loop region occupying positions one, three, four, five, six, seven and nine. The carboxyl-terminal prolinamide at position 32 and the glycine, at position 28 , are the two remaining constant positions.

In the porcine, bovine, and ovine calcitonins, a marked similarity in sequence occurs in the positions between 10 and 27; however, a noticeable difference exists between these three calcitonins when compared to human and salmon calcitonin which also differ from each other considerably. Habener et al. (1971) have suggested the increased potency of the salmon CT as compared to porcine CT may be due to an increased resistance to metabolic destruction. These in-vestigators were able to demonstrate that a progressive decrease in immunoassayable activity of porcine CT occurred after in vitro incubation of the preparation in dog plasma while salmon CT remained completely stable. Salmon CT differs from porcine CT in 19 of 32 residues, and in 11 positions it contains amino acids not found in any other CT. These structural differences might well be involved in a lesser inactivation of the molecule in the extravascular compartment, vascular bed, or one or more organs, and thereby result in greater stability of the salmon CT molecule.

Sieber et 2l. (1959) evaluated the biological activity of a variety of synthetic peptide fragments of porcine CT. Their studies indicated that fragments of the molecule, the peptide sequence 1-9 from the amino terminal of the molecule, which includes the disulfide 
bridge, or the peptide sequence 10-32, and a number of other peptide variants such as the residues 1-10 jointd to residues 20-32, exhibited no or little biological activity. Deleting even one or two amino residues from the carboxyl-terminal end of the porcine molecule while retaining the carboxyl terminal prolinamide, resulted in almost complete loss of biological activity.

Fragments of many other peptide hormones exhibit full or partial biological activity. For example, the peptide sequence, 1 through 29, of PTH exhibits full biological activity. However, the entire 32 amino acid chain together with the carboxyl terminal amide must remain intact or a dramatic decrease of hypocalcemic activity of the CT molecule occurs.

Although small variations in the length of the CT molecule drastically decrease biological activity, considerable amino acid substitution or modification within the 32 amino acid chain have little influence on biological activity, as shown by Potts et al. (1971).

\section{SITES AND MODE OF ACTION}

The principal, physiologically important action of calcitonin is the inhibition of bone resorption thereby producing its characteristic hypocalcemic and hypophosphatemic effects. Considerable evidence has been presented which indicates that calcitonin inhibits PTH-induced bone resorption both in vivo and in vitro.

The hypocalcemic effect of calcitonin is the primary attribute which resulted in its discovery in rat thyroid glands by Hirsch et al. (1963); however, Kenny (1964) and Hirsch et al. (1964) soon discov- 
ered that injections of porcine CT preparations into rats also resulted in a significant hypophosphatemic response. The decreases in plasma calcium and phosphate usually occurred over the same time interval and exhibited similar dose-response relationships. The hypophosphatemic effect of CT is now attributed to the primary action of $\mathrm{CT}$ in inhibiting bone resorption since hypocalcemia induced by CT probably results in a compensatory increase in PTH secretion. The increased PTH levels would then lead to decreased reabsorption of phosphate by the kidney and hence a hypophosphatemic response. In vivo investigations relying on administration of radioactive bone-seeking iostopes have been utilized to evaluate the mechanism of action of $\mathrm{CT}$ on bone. In order to interpret data from these types of experiments, one would have to accept the concept that there are both stable pools of calcium in bone, which are PTH-sensitive, and labile, or readily exchangeable, pools of bone calcium. Twerty-four hours after the administration of ${ }^{45} \mathrm{Ca}$, the isotope is interchanged to a great extent with the labile pools of bone calcium. Consequently, the specific activity of the radioisotope in the blood declines rapidly in the first 24 hours since there is little reabsorption of ${ }^{45} \mathrm{Ca}$ during this time interval. However, two weeks after injection of ${ }^{45} \mathrm{Ca}$, when all bone pools are labeled, alterations in PTH levels change the ${ }^{45} \mathrm{Ca}$ concentration in blood proportionately to changes in total serum calcium, thereby resulting in no change in specific activity of serum calcium. Milhaud and Moukhtar (1966) injected a single tracer dose of ${ }^{45} \mathrm{Ca}(50 \mu \mathrm{Ci})$ intravenously into rats two hours after the first of a series of injections of calcitonin which produced a low plasma calcium level for three days. The 
rats in their study, were fasted and any calcium absorption from the gut was negligible. Their studies suggested that the dilution of the radioactive calcium pool by unlabeled calcium could only be due to bone catabolism since they found that following injection of the hormone, there was the expected hypocalcemic response; however, the specific radioactivity in the blood remained constant. Therefore, Milhaud and Moukhtar (1966) proposed that the action of CT was on the stable or slowly exchangeable pool of bone rather than the labile pool which would have exhibited a drastic decline in specific activity as well as the hypocalcemic response.

Johnston and Deiss (1966) noted the effect of CT injections on the levels of radioactive calcium in rats whose bones were labeled with ${ }^{45} \mathrm{Ca}$ either three hours or two weeks before CT injection. CT produced a significant decrease in labeled as well as unlabeled calcium (no change in specific activity) in serum of rats which had received $40 \mu \mathrm{Ci}$ of ${ }^{45} \mathrm{Ca}$ prior to hormone injection. However, in three-hour labeled rats (receiving $10 \mu \mathrm{Ci}$ ) in which stable bone was not labeled, serum calcium also decreased but there was an increase in serum calcium specific activity. Their experiments indicated that CT causes inhibition of the metabolically active bone resorption.

Klein et al. (1967) studied the effect of porcine CT in rats by utilizing a peritoneal lavage technique which employs a calciumand phosphate-free rinse. ${ }^{45} \mathrm{Ca}$ and ${ }^{32} \mathrm{P}$ were administered to rats either three weeks or 18 hours before peritoneal lavage. The removal of ${ }^{45} \mathrm{Ca}$ and ${ }^{32} \mathrm{P}$ administered 18 hours prior to peritoneal lavage of normal and parathyroidectomized rats was decreased by CT as was 
that of total calcium and phosphate; however, CT decreased the removal of the isotopes from bone to a greater degree in rats labeled three weeks prior to lavage. In concurrence with other investigators, their results indicated that the labile calcium in bone as well as the stable calcium was affected by $C T$, but to a lesser degee. O'Riordan and Aurbach (1968) presented evidence that CT caused no detectable change in the disappearance of radioactive calcium from rats injected with ${ }^{45} \mathrm{Ca} 2.5$ hours earlier although serum calcium levels fell precipitously and there was an interruption in the normal decline in specific activity. Their data implied that CT had inhibited further dilution of radioactive calcium in the circulation with unlabeled calcium by blocking the mobilization of calcium from bone.

The most direct evidence indicating an effect of CT on bone in vivo was that presented by MacIntyre et al. (1967). These investigators demonstrated that $\mathrm{CT}$ infusion caused a net retention ( $+5 \%)$ of calcium in the isolated perfused tibia of the cat. This retention was associated with a small increase in acidity of the outflowing venous blood, possibly indicative of the formation of a calcium phosphate salt with release of hydrogen ion. In other in vivo experiments, the rate of urinary excretion of hydroxyproline, a major component of collagen and hence, of the bone matrix, has been utilized as an indicator of the rate of matrix breakdown, and indirectly, of bone resorption. In line with this concept, Harris and Sjoerdsma (1966) demonstrated that $\mathrm{P}^{\mathrm{m}} \mathrm{H}$ injection into rats which had received isotopically labeled L-proline four to twelve weeks earlier, increased the urinary hydroxyproline output, indicating 
destruction of mature collagen. Martin et al. (1966) demonstrated that infusion of $20 \mathrm{\mu g}$ per hour of CT dramatically decreased the excretion of hydroxyproline in intact rats after a three hour period. similarly, Rasriussen et al. (1967) have demonstrated that CT, administered in conjunction with $\mathrm{PTH}$, could inhibit the rise in urinary hydroxyproline in thyroparathyroidectomized rats. The effects of CT administration on extracellular levels (serum and lavage fluid) of hydroxyproline in rats were examined by Klein and Talmage (1968) following peritoneal lavage, parathyroidectomy, nephrectomy, and calcium-restricted diet. These investigators noted that CT markedly reduced hydroxyproline levels in all instances, being especially efficacious in reducing the total amounts of hydroxyproline and calcium removal during an 8-hour lavage period with a calcium and phosphate free rinse. The foregoing in vivo experiments provided substantial evidence that $\mathrm{CT}$ administration resulted in inhibition of all phases of bone resorption.

In vitro experiments have been of two kindss those in which the effect of CT has been studied on cultured bone and those in which changes in an isolated, perfused limb have been investigated. Alipoulious et al. (1966) produced resorption in cultured calvariae from five day old mice by the daily addition of 0.5 units of PTH to the in vitro culture system over a seven day period. The PTH-induced resorption of cultured bones had previously been well established by Gaillard (1965). However, these investigators noted that CT added to tissue cultures of calvariae could counteract, in a doserelated manner, the resorption of bone stimulated by daily PTH additions. Similarly, Friedman and Raisz (1965) and Friedman et al. 
(1968) noted the effectiveness of CT in inhibiting PTH-induced bone resorption of radii and ulnae obtained from 19-day fetal rats whose mothers had been injected with ${ }^{45} \mathrm{Ca}$ on the $17^{\text {th }}$ day of gestation. The inhibition of bone resorption was independent of the action of PTH since CT could also be shown to inhibit spontaneous resorption which occurred in control bone cultures in the absence of PTH. The inhibitory effect of $\mathrm{CT}$ on bone resorption could not be overcome by increased PTH doses. However, the inhibition in tissue culture appeared to be of limited duration. Friedman et al. (1968) and Wener et al. (1972) demonstrated that after a period of 36 to 48 hours of treatment with both PTH and CT, the cellular transformation and the elevated ${ }^{45} \mathrm{Ca}$ release, characteristic of PTH-induced bone resorption in tissue culture, reappeared despite the addition of fresh CT in maximal or supra-maximal doses. However, fresh additions of CT to culture media after several days without CT resulted once again in active inhibition of resorption. Wener and coworkers demonstrated that the initiation and magnitude of this "escape" phenomenon was proportional to the intensity of stimulation of bone resorption. Concentrations of PTH which produced submaximal stimulation of bone resorption either delayed the onset of "escape" or resulted in "incomplete" escape. Further, these investigators showed that a 24 hour pre-incubation of bones with PTH could result in a prolonged resorptive response which they termed "induction." This inductive response occurred even though the bones were washed and transferred to fresh media without PTH and with or without CT. Low concentrations of calcium or phosphate in the culture medium impaired "induction" and also impaired or delayed "escape." The 
mechanisms of the "escape" and "induction" phenomena are unknown, but it is likely that both depend upon shanges in nuclear transcription which leads to an increase in the activity and number of bone resorbing cells. Brand and Raisz (1972) carried out bone culture studies which indicated that PTH stimulates the release of both previously incorporated ${ }^{45} \mathrm{Ca}$ from the mineral and $3_{\mathrm{H} \text {-hydroxyproline }}$ from the matrix of fetal rat bone. Moreover, their studies suggest that mineral breakdown must precede matrix breakdown, since the ${ }^{45} \mathrm{Ca}$ label was lost earlier and at a more rapid rate than $3_{\mathrm{H} \text {-hydroxypro- }}$ line. The addition of 2 or $3 \mathrm{mM}$ phosphate to the incubation medium provided sustained inhibition of PTH-induced resorption of matrix as well as mineral. However, CT addition prompted inhibition of mineral resorption which was short-lived and which was not effective

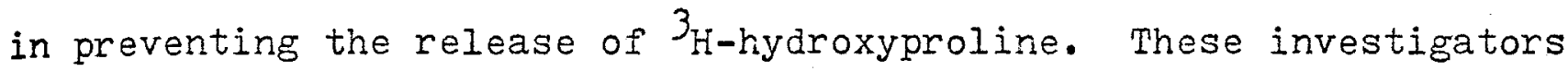
proposed that the ineffectiveness of $\mathrm{CT}$ in blocking matrix resorption was attributable to the inability of the hormone to block release of collagenases into the culture medium after PTH exposure. Thus, Brand and Raisz proposed that CT has no direct effect on the activity or synthesis of PTH-induced collagenolytic enzymes, but was effective in blocking resorption of calcium from bone mineral. The preceding in vitro experiments indicate that bones undergoing PTH-induced resorption are more responsive to additions of CT. However, Munson et al. (1966) and Gudmundsson et 2l. (1966) clearly indicated that $\mathrm{CT}$ does not act by direct inhibition of $\mathrm{PTH}$, since its unique hypocalcemic response could be demonstrated in parathyroidectomized rats which remained normocalcemic by feeding of a high calcium diet. 
The in vitro bone culture experiments of Raisz and Niemann (1967) and Reynolds and Dingle (1968) indicate that the effectiveness of CT in inhibiting PTH-induced bone resorption may be sufficiently rapid to account for the hypocalcemic effect in vivo. Raisz and Nieman's in vitro culture system consisted of paired radii and ulnae from 19-day old rat fetuses which had been prelabeled with ${ }^{45} \mathrm{Ca}$ on the seventeenth day of gestation. Their studies indicated that CT adition to the culture medium could effectively inhibit release of ${ }^{45} \mathrm{Ca}$ from $\mathrm{PTH}-$ stimulated bones within three hours. Reynolds and Dingle labeled calvariae of two day old mice with ${ }^{45} \mathrm{Ca}$ and removed the labeled calvariae for tissue culture six days after birth. The PTH-induced resorption of these calvariae could be significantly suppressed by CT within 30 minutes after the addition of the hormone.

Nisbet and Nordin (1967), utilizing an in vitro bone culture system consisting of calvariae from 5-day old mice, demonstrated that these PTH-treated calvariae released more citrate while CT and PTH addition reduced the levels of citrate and of calcium in the medium. These investigators noted that the PTH induced inhibition of metabolism of $1,5-{ }^{14} \mathrm{C}$-citrate was not affected by CT. It was possible that CT was exerting its inhibitory effect on citrate accumulation in the medium by blocking a step in glucose metabolism before citrate. Even though this possibility existed, a popular concept of the primary action of PTH was thought to be that of increasing the citrate concentration at the bone/blood interface and that a rise in citrate concentration was the initial event in PTHinduced mobilization of calcium from bone. However, this concept 
of the contrasting metabolic actions of PTH and CT when closely examined, was not tenable since Firscher et al. (1959) had demonstrated that a rise in citrate concentration in intact dogs after PIH-induced rescrption was accompanied by a concomitant rise in lactate concentration. It is now clear that changes in citrate and lactate do not precede mobilization of calcium from bone, but may well be secondary to changes in calcium concentration.

Reynolds (1968) induced resorption of near term (19-day) mouse calvariae, grown in vitro, by using vitamin A (22 international units of retinol/ml of medium) and PTH. Vitamin A and PTH treatment for two days induced resorption of calvariae with the appearance of numerous multinucleated osteoclasts. Moreover, most recognizable osteocytes disappeared, and a transformation of osteoblasts into a fibroblastic form occurred when CT was added to the culture media with vitamin A or PTH; bone resorption was suppressed since, histological sections showed a decline in the number of multinucleated osteoclasts. However, CT did not prevent the PTH and vitamin A-induced loss of osteocytes, or the transformation of osteoblasts. These investigators therefore proposed that CT primarily inhibited in vitro resorption of calvariae by altering the number of multinucleated osteoclasts.

In contrast to the work of Reynolds (1968), the in vivo work of Belanger and Rasmussen (1968) demonstrated that CT inhibited bone resorption (PTH-induced bone resorption was thought to occur through a process termed "osteocytic osteolysis" by Belanger in 1965) in thyroparathyroidectomized rats infused with $\mathrm{PTH}$ and $\mathrm{CT}$ for 14 to 16 hours. These investigators noted changes in the cellular 
structure of osteocytes and chemical changes in the matrix immediately adjacent to the lacunae containing osteocytes within four hours of increased PTH activity or after long term treatment with low calcium diets, hypervitaminosis $D$, pregnancy, or parathyroid tumor. Histochemical studies revealed that osteocytes released alkaline phosphatase and Iysosomal proteases. Similarly, there was a reduction in mineral and matrix content around the osteocyte as well as an increase in stainable mucopolysaccharide. The lacunae became enlarged with irregular borders, indicating increased resorptive activity. However, CT-treated rats showed a reversal of some of these processes since there were fewer enlarged lacunae and the matrix was more dense.

In further in vitro experiments with rat calvaria, Reynolds et al. (1968) demonstrated that vitamin A, in a manner similar to that of PTH, increased release of lysosomal enzymes. This release was not blocked by $\mathrm{CP}$ even though vitamin $\mathrm{A}$-induced resorption (increased multinucleated osteoclastic activity) was halted. These investigators proposed that CT acted by inhibiting the release of bone mineral from resorbing bone and preventing the breakdown of mature collagen. However, the hormone was not thought to affect the turnover of other matrix components (hexosamine and hydroxyproline) from immature collagen of embryonic bones which are degraded by the release of $\mathrm{PTH}$ and vitamin A-induced lysosomal enzymes.

On the other hand, there was abundant evidence based on tissue culture studies of Gaillard and Goldhaber and peritoneal lavage experiments of Talmage and coworkers that implicated the osteoclast in mediation of PTH-induced bone resorption. Gaillard (1965) had 
shown that administration of $\mathrm{PTH}$ to cultured mouse radii resulted in decreased osteoblastic activity in bone shafts and increased numbers of multinuclear osteoclasts in areas of active bone resorption. Goldhaber (1965), utilizing the calvaria of 5-day old mice, demonstrated the appearance of numerous giant vacuolated osteoclasts (40-50 $\mu$ in diameter) which developed and accumulated in areas of rapid resorption 24 hours after PTH or vitamin D-treatment.

The peritoneal lavage studies of Talmage (1967) and Park and Palmage (1968) confirmed the stimulation of osteoclast production following FTH-treatment. However, their experiments indicate that these effects on osteoclasts do not correlate in time or location with the rapid mobilization of calcium from bone due to PTH-treatment since stimulation of osteoclast production did not occur until six to eight hours after PTH administration. These investigators proposed that both mechanisms of bone resorption, osteocytic and osteoclastic, are physiologically important. Park and Talmage proposed that osteocytic osteolysis may be the principal mechanism for rapid mobilization of calcium from bone in response to PTH-treatment and the osteocytes are the bone cells which are responsive to cr-treatment. The action of PTH on osteocytes did not appear to require increased RNA synthesis as a prerequisite for hormone action. These investigators propose that the osteoclast plays a prominent role in the normal remodeling of bone and the chronic, or longer term effects of continued PTH-induced bone resorption. The production of increased numbers of osteoclasts may proceed through changes in RNA synthesis in mesenchyme cells, the progenitors of osteoclasts. 
Although the gastrointestinal tract has an important influence on the extent of absorption of dietary calcium, and to some extent, the levels of calcium excretion, it is not critical in mediating the hypocalcemic effect of CT. Aliapoulious et al. (1965) and Munson et al. (1.966) have clearly demonstrated that CT was effective in lowering blood calcium levels of rats even after removal of the entire gastrointestinal tract. Additional evidence pointing to the lack of $\mathrm{CT}$ involvement in modulating calcium absorption was presented by Robinson et al. (1968) who demonstrated that CT had no influence on calcium absorption from isolated gut loops of rats. The other organ system which is important in calcium metabolism, the kidney, was also shown by Munson et a.l. (1966) and Gudmundsson et al. (1966) not to be essential in mediating the action of CT. These investigators shawed that CT was effective in lowering blood calcium levels of rats when given immediately after nephrectomy, indicating the fall in plasma calcium was not due to increased renal excretion of calcium.

\section{MECHANISM OF ACTION}

Currently, there exists no convincing data suggesting an explanation of the biochemical mode of action of CT in inhibiting PTH-induced bone resorption as mediated either by the osteocyte or the osteoclast bone cells in vivo or in vitro. However, it has been clarified that the hormone's hypocalcemic effect is not dependent upon direct inhibition of PTH. This view was well documented by the studies of Tashjian (1965) and Robinson et al. (1967) who 
demonstrated that CT was fully active in surgically parathyroidectomized rats. It is unlikely that CT administration affects provein synthesis, or the formation of new RNA since the action of the hormone is unaffected by prior administration of actinomycin $D$ to rats as demonstrated by Tashjian (1965) and Gudmundsson et al. (1966). Tashjian's work indicated an unaltered hypocalcemic response in rats which had been injected with actinomycin $D, 3,8$, or 11 hours prior to CT administration. The hypocalcemic effect could be observed at 8 to 12 hours after actinomycin $D$ injection when endogenous or exogenous PTH proved completely ineffective. Moreover, the action of $\mathrm{CT}$ is not dependent on vitamin $\mathrm{D}$, since the hormone exerts its hypocalcemic and hypophosphatemic effect in vitamin D-deficient rats as shown by Morii and DeLuca (1967).

Chase and Aurbach (1967 and 1968) and Chase et al. (1969) presented experimental evidence that suggested that cyclic $3^{\circ}, 5^{\circ-}$ adenosine monophosphate (cyclic AMP) was a mediator in the mechanisin of action of PTH on the kidney and bone in vitro. PTH stimulated adenyl cyclase, the enzyme which converts ATP to cyclic AMP, in rat renal cortex preparations within 15 seconds after in vitro hormone addition. In line with Chase and Aurbach's work, Wells and Iloyd (1967 and 1968) have advanced the hypothesis that CT exerts its characteristic hypocalcemic and hypophosphatemic effect by altering the activity of the enzyme, phosphodiesterase, which degrades cyclic AMP. Their hypothesis was based on indirect evidence derived from utilizing the Fharmacologic agents, imicazole, isoproterenol, and theophylline. According to their hypothesis, agents which activate phosphodiesterase should have a hypocalcemic action similar to that 
of CT. Wells and Lloyd (1968) found that injection of imidazole, which stimulates phosphodiesterase activity, led to hypocalcemia in intact, parathyroidectomized or thyroparathyroidectomized rats, thereby mimicking the action of CT. On the other hand, administration of large doses of theophylline, (Wells and Lloyd, 1967) which was thought to act by diminishing the degradation of cyclic AMP, and isoproterenol (Wells and Iloyd, 1968) which enhances adenyl cyclase and consequently, cyclic AMP synthesis, should affect serum calcium levels in a similar fashion to PTH. These investigators found that the two agents were effective in blocking the hypocalcemic effect of exogenous or endogenous CT in intact and parathyroidectomized rats. Based on these observations, Wells and Lloyd hypothesized that the rate of mobilization of calcium from bone and, secondarily, the level of blood calcium, are related to variations in the level of cyclic AMP within bone cells. The hypercalcemic effect of parathyroid hormone was thought to depend on its ability to elevate bone cell cyclic AMP by enhancing bone adenyl cyclase activity while the hypocalcemic effect of $\mathrm{CT}$ might be due to the ability of this agent to lower cyclic AMP levels by activating phosphodiesterase activity.

Wells and Lloyd's hypothesis is no longer tenable, since Care et al. (1971) have demonstrated that a diacylated derivative of cyclic AMP, dibutyryl cyclic AMP, stimulated CT secretion in vivo in pigs, and that this stimulatory effect was shared by theophylline, a recognized inhibitor of endogenous degradation of cyclic AMP. In addition, Chase and Aurbach (1970) and Murad et al. (1970) found that porcine CI caused small increases (about two-fold) in concen- 
tration of cyclic AMP in bone and in kidney homogenates in vitro (Murad et al., 1970). These investigators demonstrated that there was Iittle or no effect of dose, whereas PTH showed a log-dose response in this system over a ten to twenty-fold range of cyclic AMP concentration. Moreover, Chase and Aurbach demonstrated that CT did not activate adenyl cyclase or affect phosphodiesterase levels in this tissue. Therefore, it would be difficult to conceive that the action of both PTH and $\mathrm{CT}$, recognized as physiological antagonists, could be mediated through increased cyclic AMP concentrations in the same cell. The foregoing experimental observations tend to indicate that whatever the mechanism of action of CT on bone, it most likely does not occur at the level of cyclic AMP effects, but rather antagonizes the action of PTH by another, as yet, undetected subcellular mechanism. The observations of Wells and Lloyd could thus be explained simply as another example of the overall physiological antagonism of PTH and CT in maintaining mineral homeostasis. Another set of biochemical observations in which CT has been implicated are the effects of PTH and CT in, respectively, inllibiting and stimulating the activity of bone pyrophosphatase ( $P$-Pase). Fleisch et al. (1966) have proposed that inorganic pyrophosphate (PPi), an anhydrodimer of orthophosphate, might be one of the physiological regulators of calcification. These investigators proposed that calcium phosphate deposition can occur only after the destruction of pyrophosphate by pyrophosphatase (alkaline phosphatase), an enzyme present in large concentration in calcifying tissues. Fleisch and coworkers demonstrated that addition of PPi inhibited the calcification of chick femurs in tissue culture, an observation which 
might be explained as a blockage of crystal growth centers by adsorption of PPi onio apatite crystals at the site of calcification. Their studies indicated that pyrophosphate-coated crystals dissolved more slowly. The physiological importance of this observation was uncertain; however, it is possible that CT might diminish P'Ifi-induced bone resorption through a mechanism of increasing the protective PPi layer.

orimo et al. (1971) demonstrated that CT caused a significant and rapid increase in alkaline phosphatase activity of rat tibia within 15 minutes after injection. The increased alkaline phosphatase level preceded the development of a significant hypocalcemia. PTH injection into thyroparathyroidectomized rats significantly decreased the alkaline phosphatase activity of tibia within six hours. These investigators have suggested that $C T$ activates alkaline phosphatase which consequently degrades PPi and subsequently may facilitate bone mineralization or accretion. Bone resorption may occur through PTH-induced activation of acid phosphatase. Their experiments utilizing physiological concentrations of PTH and CT suggested that the various pyrophosphatases were directly involved in the mechanism of action of $\mathrm{CT}$ and PTH.

Rasmussen and Tenenhouse (1967) and Tenenhouse and Rasmussen (1968) have utilized an in vitro system consisting of Ehrlich ascites tumor cells. This would allow them to study a homogeneous cell population incubated in a chemically defined medium, which would be responsive to $\mathrm{CT}$ and $\mathrm{PTH}$ in an easily detectable fashion, and the hormonal. response might be likened in some way to the in vivo action of these two hormones. These investigators harvested the tumor cells 
and incubated them with $\mathrm{CT}$ and $\mathrm{PTH}$. The response was either removal of ${ }^{45} \mathrm{Ca}$ from dead, prelabeled mouse bone, or P-Pase activity. parathyroid hormone, in the presence of $10^{-4} \mathrm{M} \mathrm{Ca}{ }^{++}$, increased the release of ${ }^{45} \mathrm{Ca}$ from the bone, increased cyclic AMP concentration in the tumor cells, and inhibited the activity of P-Pase which was released into the medium (Rasmussen and Tenenhouse, 1967). CT stimulated pyrophosphatase activity in the absence of PTH and removed the blocking effect of PTH on P-Pase. However, Tenenhouse and Rasmussen (1968) demonstrated that CT did not inhibit the PTHinduced increase in cyclic AMP within these cells.

Although a number of interesting hypotheses explaining the biochemical basis of action of both $\mathrm{CT}$ and $\mathrm{PTH}$ in regulating mineral homeostasis have been advanced in recent years, none have been sufficiently well-documented to date, and this still remains an area of intensive research.

\section{CONTROI OF SECRETION}

As implied by the previously cited results of Copp et al. (1962), Kumar et al. (1963), Foster et al. (1964), Talmage et al. (1965), and Care (1965), the rate of secretion of calcitonin is regulated by the calcium concentration of the blood. flowing through the thyroid gland, rising as the calcium level increases and falling as it decreases.

Care et al. (1968) examined the relationship between CT secretion rate and plasma calcium concentration in pigs in an endeavor to clarify the control mechanism of CT secretion as related to blood 
calcium. Pig thyroid glands, which have no embedded parathyroid tissue, were surgically isolated and perfused with blood, the calcium concentration of which varied between 5 to $11 \mathrm{mEq} / 1$. The thyroid venous effluent from these perfused pig thyroids was assayed for hypocalcemic activity and CT content by intravenous injection into 5 to 6 week old rats. These investigators demonstrated that there was a high correlation between plasma calcium concentration in the thyroid venous plasma and the rate of CT secretion since a linear relationship existed between these two parameters over the range of 6-10 $\mathrm{mEq} \mathrm{Ca} / 1$. It was determined that no significant change in CT secretion occurred over a 3-fold range of blood flow rates through the perfused thyroid, from which Care and coworkers concluded that the CT released by the ' $C$ ' cell of the thyroid was directly controlled by the ambient plasma calcium concentration and not by the rate, degree, or direction of calcium flux in the plasma. However, these investigators were unable to detect $\mathrm{CT}$ secretion at normocalcemic levels, below $6 \mathrm{mEq} / 1$, which they attributed to the limitations of their bioassay technique rather than to the absence of CT in normocalcemic blood. The evidence of Care and coworkers supported a direct proportional control of plasma calcium.

Klein and Talmage (1968) and Minkin and Talmage (1968) carried out a number of experiments in rats utilizing the peritoneal lavage technique which supported the concept that CT was continuously secreted throughout the normal range of plasma calcium levels ( 9 to Il $\mathrm{mg} / 100 \mathrm{ml})$. In their studies, all the rats were parathyroidectomized and half of these were thyroidectomized, and received ${ }^{85} \mathrm{Sr}$ and/or ${ }^{45} \mathrm{Ca}$ one or two weeks before experimental use. The lavage 
procedure utilizing a lavage fluid of $12 \mathrm{mg} \%$ calcium, allowed calcium transfer into the hypocalcemic rats and gradually raised the starting plasma calcium values of $7.0 \mathrm{mg} \%$ to the normocalcemic range of $10 \mathrm{mg} \%$. Although only slight differences in both plasma calcium and total transfer of calcium out of lavage fluid were observed between the parathyroidectomized and thyroparathyroidectomized rats, a marked difference developed in the amount of radioisotope transferred to lavage fluid. Rats with functional thyroids transferred 25\% less radioactivity into lavage fluid than thyroparathyroidectomized rats. These investigators interpreted this to mean that CT secretion was prompted by calcium challenge ( $12 \mathrm{mg} \%$ lavage fluid) and that CT was secreted and was effective in preventing removal of the stable pool $\left({ }^{45} \mathrm{Ca}\right.$ and $\left.{ }^{85} \mathrm{Sr}\right)$ of bone mineral, even at normal plasma calcium levels.

Although Care et al. (1968) had suggested that CT was secreted at normocalcemic ranges and the experimental evidence of $\mathrm{klein}$ and Talmage (1968) and Minkin and Talmage (1968) allowed such an interpretation, no direct experimental evidence was available which would indicate that CT was continuously secreted in the regulation of normal blood calcium concentrations. The subsequent development of the radioimmunoassay technique by Deftos et al. (1968) and Lee et al. -(1969) made possible the confirmation that the hormone is secreted continuously under normal physiological concentrations of blood calcium. Calcitonin concentration in rabbits was measured both in the peripheral circulation and thyroid effluent blood by Deftos et al. (1968). The concentration of CT found in thyroid effluent blood in unstimulated rabbits was $10 \mathrm{m \mu g} / \mathrm{ml}$ while that in the peripheral cir- 
culation was $0.14 \mathrm{m \mu g} / \mathrm{ml}$. These investigators detected rapid changes in $\mathrm{CT}$ concentration following hypercalcemic challenge. Peripheral blood concentrations of the hormone were elevated three to fifteen-fold shortly after infusions of calcium chloride. However, no CT was detected in the blood of thyroidectomized rabbits, before, during, or after the calcium challenge. Recent studies by Cooper et al. (1971) utilizing a radioimmunoassay for porcine CT clearly demonstrated that there was a rapid response in CT secretion rate, when hypercalcemia was induced by calcium chloride infusion. Hormone concentrations rose dramatically from basal levels of $10-20 \mathrm{m \mu g} / \mathrm{ml}$ in thyroid venous effluent plasma to as much as $2000-3000 \mathrm{m \mu g} / \mathrm{ml}$ when blood calcium was elevated to $15 \mathrm{mg} / 100$ ml. When hypercalcemia was eliminated by administration of EDTA, a rapid decline of $\mathrm{CT}$ occurred in thyroid venous blood and hormone was essentially undetectable as calcium fell to $8 \mathrm{mg} / 100 \mathrm{ml}$.

Radde et al. (1970) carried out experiments to determine if varying concentrations of divalent cations ( $\mathrm{Ca}^{++}$and $\mathrm{Mg}^{++}$) would stimulate the release of CT from fresh pig thyroid slices in a KrebsRinger bicarbonate buffer incubation medium. These investigators demonstrated that the levels of $\mathrm{CT}$ in the incubation media rose with increasing calcium levels in the media. A 20 to $25 \%$ increase in calcium concentration was necessary to produce enhanced CT release into the incubation media indicating that the effect of calcium ion was direct. Increasing magnesium concentrations also stimulated increased CT release, but this did not occur until magnesium levels had been raised to double the normal value (to $3.0 \mathrm{meq} / 1$ ), a situation which probably never occurs under normal circumstances; more- 
over, by measuring the residual biological activity in incubated thyroid slices, these investigators confirmed that increasing calcium concentrations increased both synthesis and release of CT.

Gittes et al. (1968) assayed thyroid glands of rats, subjected to either prolonged hypocalcemia or hypercalcemia for CT content and demonstrated that levels of $\mathrm{CT}$ within the glands were consistent with the experimentally achieved levels of blood calcium. Systemic hypercalcemia induced in intact rats by intraperitoneal injections of calcium chloride reduced the CT content of the thyroid glands to $35 \%$ of the content in normocalcemic controls as early as two hours after the onset of hypercalcemia. However, recovery to normal CT levels in the gland occurred within sixteen hours after cessation of a two hour hypercalcemia. In parathyroidectomized rats fed a normal diet, a chronic hypocalcemia occurred with concomitant 12-fold accumulation of CT over normal levels within 14 weeks. Rats which received parathyroid homografts returned to normocalcemic ranges, although the CT content of the glands was not markedly reduced. Gittes and coworkers concluded that systemic hypercalcemia was the stimulus for rapid release of $\mathrm{CT}$; however, the rate of $\mathrm{CT}$ biosynthesis was not sufficiently increased to maintain an elevated rate of secretion. During chronic hypocalcemia, minimal amounts of stored CT are secreted, although hormone biosynthesis remains unaltered and consequently more CT was stored in the glands. The studies of Deftos et al. (1968), Lee et al. (1969), and Cooper et al. (1971) confirmed the observations of Care et al. (1968) that the rate of CT secretion was directly related to the degree of hypercalcemia. Moreover, these studies demonstrated that CT was 
continuously secreted and that the rate of hormone secretion was under directly proportional control of blood calcium even in the normocalcemic range.

It is currently well-established that blood calcium concentration is under reciprocal control of PTH and CT. Hypercalcemia stimulates CT secretion and inhibits PTH secretion; this change leads to a reduction in the rate of bone resorption and a consequent decline in blood calcium concentration. Conversely, hypocalcemia inhibits CT secretion and stimulates PTH secretion; the resultant increase in bone resorption provides sufficient calcium from skeletal mineral deposits to restore blood calcium concentration toward normal levels.

\section{METABOLISM AND DURATION OF ACTION}

Scant information exists concerning the metabolic fate of calcitonin. Deftos et al. (1968) injected a rabbit with a potent extract of porcine CT ( 90 MRC units) and monitored the disappearance of the hormone from the peripheral circulation utilizing their radioimmunoassay technique. Although CT concentrations were as high as $500 \mathrm{mU} / \mathrm{ml}$ ten minutes after hormone injection, the levels dropped precipitously to $0.6 \mathrm{mU} / \mathrm{ml}$ after one hour. On the basis of this observation and from the decline of endogenous CT which occurred in rabbits after the cessation of calcium chloride infusion, these investigators proposed that the circulating half-life of the hormone was approximately 10 to 30 minutes. Foster et al. (1972) demonstrated that the rate of clearance of CT from blood was extremely 
rapid. These investigators have established by use of radioimmunoassay that human CT injected into dogs disappeared rapidly having an initial half-life of three minutes which was followed by a second, more prolonged half-life of 40 minutes. Injections into dogs of calcitonin labeled with ${ }^{13}{ }^{1}$ demonstrated that the hormone was mainly concentrated by the liver and kidney. Although both of these organs concentrated radioiodinated human $\mathrm{CT}$, Foster and coworkers demonstrated that nephrectomized dogs exhibited higher levels of circulating hormone and that the initial disappearance of the hormone was prolonged. Similarly, arterio-venous differences across the dog kidney following infusion of human $\mathrm{CT}$ indicatad that nearly one-third of the infused hormone was removed whereas only minor amounts were removed following liver infusion. Although these studies indicated that the circulating half-life of CT was of short duration ( 3 to 30 minutes) and that the kidney played a role in hormone degradations, no studies have been carried out or hypotheses advanced which endeavor to explain the rather long duration of the hormone's hypocalcemic effect.

\section{ROLE IN CLINICAL DISORDERS OF CALCIUM METABOLISH}

Since it has been thoroughly established that calcitonin inhibits bone resorption both in vitro and in vivo and thereby lowers blood calcium concentrations, the hormone might be efficacious in the treatment of both hypercalcemic conditions and diseases involving increased bone resorption. 
A number of experimenters have successfully lowered blood calcium levels with CT in hypercalcemic patients. Foster et al. (1966) were the first investigators to utilize porcine CT as a therapeutic agent in the treatment of three patients with hypercalcemia as a result of metastic carcinoma of bone. Calcitonin injection at a dose level of 1 to $22 \mathrm{MRC}$ units, resulted in a marked reduction in serum calcium levels for periods up to 18 hours, although serum calcium values never quite reached normal levels. Haas and Dambacher (1968) observed remissions of hypercalcemia in 12 patients with hyperparathyroidism which were injected with porcine calcitonin. Milhaud (1968) demonstrated that children with hypercalcemia resulting from hypersensitivity to vitamin $D$ or vitamin $D$ intoxification responded well to injections of $0.1 \mathrm{NRC}$ units calcitonin by an enhanced and prolonged hypocalcemia which was manifested one hour after hormone injection.

The syndromes, hypervitaminosis $D$, hyperparathyroidism, and metastic carcinoma of bone, all involve extensive resorption of bone and should therefore prove amenable to the therapeutic use of calcitonin.

A number of investigators have also attempted to investigate the effects of CT injection in preventing bone resorption in several types of experimentally-induced osteoporosis. Foster et al. (1968) produced experimental osteoporosis in rats by subcutaneous injections of 2500 international units of vitamin A three times a week. It was found that $100 \mathrm{mo}$ of CT administered over a four-week period not only prevented vitamin A-induced resorption, but also retarded some of the general systemic effects produced by vitamin A. Experi- 
mental conditions similar to osteoporosis were produced in rats by Fujita et al. (1968) by feeding the animals low calcium diets and by treatment with corticosteroids. Tibia of rats treated with the corticosteroid, prednisolone, were decreased in thickness and were less resistant to bending stress than control rats or animals which had received CT injections concurrent with corticosteroid treatment and low calcium diet. However, the possibility that CT might prove to be effective in treating human osteoporosis, especially the postmenopausal variety, remains uncertain.

Considerable therapeutic success has been achieved in patients with generalized Paget's disease. This is a syndrome which is characterized by a rapid rate of bone turnover without hypercalcemia, increased urinary hydroxyproline excretion, and elevated serum alkaline phosphatase. Bijvoet et al. (1968) noted a marked decline in plasma calcium, phosphate, and hydroxyproline of patients with generalized Paget's disease subsequent to injections of small doses of porcine CT. It was likely that CT administration was efficacious in retarding the rate of bone turnover.

\section{PHYSIOLOGICAL ROLE}

Despite all that has been learned about calcitonin since its postulated existence by Copp et al. (1962), and discovery in the thyroid by Hirsch et $\underline{\text { l. }}$ (1963), its physiological role in the maintenance of vertebrate mineral homeostasis remains uncertain. In response to an adequate stimulus, PTH-induced hypercalcemia, CT is secreted by the thyroid gland into the general circulation where the hormone markedly retards bone resorption as evidenced by the 
rapid decline in blood calcium levels. However, calcitonin may also play a key role in combatting hypercalcemia of dietary origin, although it is well known that starvation or calcium deficiency are more commonplace, natural occurrences than calcium excess.

Cooper et al. (1970) showed that endogenous CT could adequately protect rats against a hypercalcemic challenge which did not evoke a detectable rise in blood calcium in animals with intact thyroid glands. This was demonstrated by injecting sufficient PTH to elevate the serum calcium levels of thyroparathyroidectomized rats about $3 \mathrm{mg} / 100 \mathrm{ml}$ whereas sham-operated rats which had been given identical doses of PTH exhibited normal blood calcium ranges. However, thyroidectomizing half of the sham-operated rats within three hours after PTH administration prompted a persistent hypercalcemia which was no longer opposed by the action of endogenous CT. Munson and Grey (1970) demonstrated that thyroidectomized and thyroparathyroidectomized rats became transiently hypercalcemic (60 to $90 \mathrm{~min}$ utes) after a gavage of $10 \mathrm{mg}$ of calcium chloride whereas sham-operated rats exhibited unaltered blood calcium concentrations. Moreover, these investigators noted that feeding as little as three $\mathrm{mg}$ of calcium chloride by stomach tube markedly increased serum calcium levels after thyroidectomy. In an attempt to simulate natural conditions, fasted thyroidectomized rats were permitted to eat food containing a normal concentration of calcium (1\%) over a 90-minute period, and it was demonstrated that very similar results occurred. These observations of Munson and Grey provided the first direct physiological data which indicated that CT was functioning at normocalcemic ranges to protect rats against hypercalcemia. Thyroidec- 
tomized (calcitonin deficient) rats were unable to cope with hypercalcemia of naturally occurring dietary origin, this deficiency state being directly attributable to the loss of this hypocalcemic hormone. However, it was possible that the importance of CT in combatting hypercalcemia may be confined to the actual time of food consumption or immediately thereafter.

In order to fully substantiate the concept that CT is a hormone which exerts a tonic effect at normal blood calcium concentrations, it would be necessary to demonstrate spontaneous hypercalcemia in thyroidectomized animals since parathyroidectomy has been shown to result in spontaneous hypocalcemia. However, such an effect has not yet been demonstrated, and no initial hypercalcemic signal which would stimulate calcitonin release has been detected. However, Cooper and coworkers (1970) carried out an experiment which provided direct evidence that such a signal actually occurred. These investigators demonstrated that elevation in serum calcium of sham-operated rats could not be detected three hours after injection of 85 units of PTH; although, at this time interval, thyroidectomized rats which had received an identical dose of PTH were markedly hypercalcemic. Moreover, thyroidectomy of sham-operated (PTH-treated) rats three hours after PTH injection, while they were still normocalcemic resulted in elevation of serum calcium similar to rats which had been thyroidectomized at time zero. These results were further indirect proof that the presence of the thyroid gland (endogenous CT secretion) had prevented a rise over the first three hours in serum calcium due to PTH injection. 
Some experimental evidence exists that indicates CT may also influence the rate of mineral accretion. Foster et al. (1966) demonstrated that CT increased the metaphyseal area of trabecular bone in vertebra of growing rats and diminished the number of osteoclasts. Since the metaphyseal area of all bones exhibit rapid turnover rates, these investigators proposed that this is the area of bone which may serve as a ready source of calcium. Therefore, CT may prevent cortical mineral loss during periods of calcium deficiency by augmenting metaphyseal bone reserve.

Although the action of CT does not depend on the presence of the kidney, Bijvoet et al. (1971) have presented evidence that infusions of synthetic human $C P$ into humans resulted in a transient increase in the urinary excretion of sodium. This was attributed to a decrease of fractional reabsorption from the proximal kidney tubules. These investigators suggest that CT in physiological concentrations exerts a natriuretic effect sufficient to influence volume homeostasis (decreases expanded extracellular volumes) in man, an effect that endogenous CT may also bring about.

Investigations attempting to determine the exact physiological role of CT are still being actively pursued. However, evidence is accumulating that suggests calcitonin is continuously being secreted and exerting a tonic or fine control in maintaining normal blood calcium levels in healthy animals and keeping PTH-induced bone resorption at minimal levels. It is likely that CT has a more significant role in vertebrate mineral homeostasis in young, rapidly growing animals, during pregnancy, lactation, bone-healing processes, and periods of starvation or limited calcium intake. 
STATEMENT OF THE PROBLEM

A number of in vitro biosynthetic studies, some of which have employed subcellular fractionation procedures, have provided valuable information about the physiological role of a number of hormones.

Brown and Ulvedal (1960) prepared subcellular fractions from the anterior pituitary lobe of pigs by differential ultracentrifugation. The hormones of the anterior pituitary were shown by these investigators to be present in separate subcellular fractions: $L H$ and STH, in the secretory granules; TSH, in the mitochondria; and $A C T H$, in the microsomes and supernatant; and FSH, in several fractions. Similarly, Hamilton and Cohn (1969) were able to demorstrate hypercalcemic activity in the $100,000 \times \mathrm{g}$ particulate fraction from bovine parathyroid tissue. Fractions obtained by differential centrifugation of pig thyroid homogenates (Bauer and Teitelbaum, 1966) and rat thyroid homogenates (Cooper and Tashjian, 1966) were assayed for the hypocalcemic activity of calcitonin. Most of the recovered activity appeared in $100,000 \times \mathrm{g}$ particulate fraction which also contained numerous secretory granules when examined by electron microscopy.

An insight into the mechanism of in vivo synthesis and release of a number of hormones has been achieved by utilizing in vitxo biosynthetic media containing either slices or homogenates of exdocrine glands and monitoring biosynthesis and release of the hormones by biological assay. Takabatake and Sachs (1964) accomplished in vitro biosynthesis of vasopressin in hypothalamic tissue, and Adiga et al. (1965), using various labeled amino acids, demonstrated the in vitro 
biosynthesis of ACTH in anterior pituitary tissue. Studies of Hamilton and Cohn (1969) and Hamilton et al. (1971) demonstrated the in vitro incorporation of several labeled amino acids into parathyroid hormone isolated from bovine parathyroid glands. Incorporation of the tracer amino acids was found to vary inversely with the concentration of calcium in the medium suggesting that the tissue retained the physiological regulatory mechanism which is characteristic of the function of the gland in vivo. When the concentration of calcium was lowered, there was an increase in radioactive PTH isolated from the tissue and the incubation medium. The identity of the newly synthesized radioactive species with PTH was established by chromatography, polyacrylamide gel electrophoresis, and rat bioassay. Sorgente (1969) utilized an in vitro biosynthetic medium containing sheep thyroid slices to establish the role of varying calcium and magnesium ion concentrations on the synthesis and release of calcitonin. The incorporation of glycine-C-14 into ovine calcitonin was directly related to the calcium concentration of the incubation medium. High calcium concentration stimulated both synthesis and release of calcitonin while varying the magnesium concentration was without effect in the calcitonin regulatory mechanism.

The experimental work to be reported in this investigation concerns a study of the hypocalcemic activity of cytoplasmic particulate fractions obtained from ovine thyroid glands in an effort to determine more precisely its localization and to define the role of calcium ion on its biosynthesis. Such a study will be useful in the further analysis of factors affecting the biosynthesis and secretion of this hypocalcemic hormone. A differential centrifugation 
procedure will be utilized to obtain mitochondrial, nuclear, and microsomal subcellular fractions from the thyroid slices. These slices have been incubated in vitro in a biosynthetic medium containing glycine-C-14. The subcellular fractions will be assayed for hypocalcemic activity in rats and the influence of low, physiological, and high calcium concentrations on the biosynthesis of the hormone in these three fractions will be determined. Standard and subcellular calcitonin preparations will be fractionated into their protein components by polyacrylamide gel electrophoresis. This will be done to establish the identity of the hypocalcemic subcellular fraction with calcitonin preparations of known potency and to determine the homogeneity of these preparations at each stage of hormone purification. An isoelectric focusing procedure will be utilized to determine the isoelectric point of subcellular and standard calcitonin preparations, thereby providing a physical constant which may serve to further characterize this hormone. Moreover, the highly sensitive resolving power of this technique will be employed to obtain milligram quantities of purified subcellular preparations exhibiting hypocalcemic activity. 
CHAPTER II

MATERIAIS AND METHODS

GENERAL EXPERIMENTAL PROCEDURE

The first part of this investigation consisted of determining the hypocalcemic activity of particulate fractions of ovine thyroid glands and the influence of low, physiological and high calcium concentrations on the biosynthesis of the hormone in mitochondrial, nuclear, and microsomal subcellular fractions obtained from sheep thyroid slices utilizing the in vitro biosynthetic medium, described by Sorgente (1969). Sheep thyroid glands were obtained fresh from the slaughter-house 10 to 20 minutes after the animals were killed. The glands with all adhering tissue were placed in ice-cold KrebsRinger bicarbonate buffer ( $\mathrm{pH} 7.4)$ containing glucose $(320 \mathrm{mg} / 100 \mathrm{ml})$ and all the amino acids of calcitonin (20 $\mathrm{mg} / \mathrm{liter}$ ) with the exception of the tracer amino acid, glycine-C-14 (UL), which was added during the incubation period. The glands were brought to the laboratory and dissected free from fat, connective tissue, and possible contaminating parathyroid tissue. Thyroid slices ranging in thickness from 0.5 to $1.0 \mathrm{~mm}$ were prepared with a Stadie-Riggs tissue slicer. Two grams of slices were transferred to each of a series of $50 \mathrm{ml}$ Erlenmeyer flasks containing $10 \mathrm{ml}$ of the Krebs-Ringer bicarbonate buffer. To each flask, $2.5 \mu \mathrm{Ci}$ of the tracer amino acid, glycine-C-14 (UL) (International Chemical and Nuclear Corp., Irvine, Calif., 
s. A. $80 \mathrm{mc} / \mathrm{mm})$, was added, gland slices were incubated for $4 \mathrm{hrs}$ at $37^{\circ} \mathrm{C}$ in biosynthetic media containing low calcium $(4.8 \mathrm{mg} / 100 \mathrm{ml})$, physiological calcium ( $11.5 \mathrm{mg} / 100 \mathrm{ml})$, and high calcium $(26.0 \mathrm{mg}$ $/ 100 \mathrm{ml}$ ) concentrations. The incubation period was terminated by placing the Erlenmeyer flasks containing the gland slices into an ice bath after which the gland slices were minced and thoroughly nomogenized.

A standard procedure of differential centrifugation was then utilized to obtain mitochondrial, nuclear, and microsomal subcellular fractions from thyroid slices obtained from the incubation media containing low, physiological, and high concentrations of calcium. The subcellular preparations were inspected for homogeneity by phase contrast and electron microscopy. In some instances, the three lyophilized fractions were assayed for hypocalcemic activity in rats without further purification. However, in the majority of experiments, the lyophilized mitochondrial, nuclear, and microsomal preparations were further purified by passage through a column of Sephadex G-100 before assaying for biological activity. The subcellular fractions were then assayed for hypocalcemic activity in 130 to $160 \mathrm{gm}$ male Holtzman rats which had been fasted for 18 hours. Radioactivity and protein content of mitochondrial, nuclear, and microsomal preparations were determined at each stage of the purification. The hypocalcemic activity of a particular fraction was estimated by determining spectrophotometrically the change in serum calcium level before, and 50 minutes after, the injection of aliquots of each of the subcellular fractions into rats. Radioactivity was measured by the use of a Beckman LS 250 Liquid Scintilla- 
tion Spectrometer and total protein was determined by a modification of the Lowry method. The difference between the hypocalcemic activity of subcellular fractions obtained from media containing high and low calcium concentrations and fractions obtained from the medium containing a physiological calcium concentration was used as an index of calcitonin biosynthesis.

In the second part of this investigation, the microsomal fraction which had consistently demonstrated hypocalcemic activity was assayed for homogeneity by polyacrylamide gel electrophoresis. Microsomal calcitonin preparations were inspected for homogeneity in polyacrylamide gels after passage through Sephadex G-100 and G-50 columns. A standard calcitonin preparation of known potency was electrophoretically fractionated along with the microsomal preparations to establish the identity of the microsomal preparation. The standard calcitonin preparation had a potency of 88 Medical Research Council Units and was fractionated along with the microsomal fraction into their component protein bands in anionic polyacrylamide gels under identical conditions of milliamperage per gel column (2.5 ma), electrophoretic separation time ( 1 hour and 20 minutes), and gel concentration ( $7.5 \%)$.

Finally, an isoelectric focusing procedure utilizing an LKB 8100 Electrofocusing Column (LKB Instruments, Inc., Rockville, Maryland) was employed to characterize microsomal calcitonin preparations, by determining their isoelectric points. The highly sensitive resolving power of this technique which is based on the amphoteric nature of protein molecules was employed to obtain milligram quantities of the component protein bands of microsomal subcellular 
preparations. The resolved protein bands were then assayed for radioactivity and hypocalcemic activity utilizing a rat bioassay. All chemicals utilized in this investigation were of analytical reagent grade.

PREPARATION OF INCUBATION MEDIA

Krebs-Ringer bicarbonate buffer with modifications of the calcium ion concentration served as the incubation medium for ovine thyroid slices. The composition of Krebs-Ringer bicarbonate buffer at a physiological calcium ion concentration ( $11.5 \mathrm{mg} \mathrm{Ca}^{++}$) $1100 \mathrm{ml})$ is represented below:

$\begin{array}{ccccccc}100 & \operatorname{part}(\mathrm{s}) & 0.90 \% & (0.154 \underline{\mathrm{M}}) & \mathrm{NaCl} \text { solution } & (0.1180 \underline{\mathrm{M}}) * \\ 4 & " & 1.15 & " & \mathrm{KCl} & " & (0.0047 \underline{\mathrm{M}}) * \\ 3 & " & 1.22 & (0.110 \underline{\mathrm{M}}) & \mathrm{CaCl}_{2} & & (0.0025 \underline{\mathrm{M}}) * \\ 1 & " & 2.11 & (0.154 \underline{\mathrm{M}}) & \mathrm{KH}_{2} \mathrm{PO}_{4} " & (0.0012 \underline{\mathrm{M}}) * \\ 1 & " & 3.82 & " & \mathrm{MgSO}_{4} \cdot 7 \mathrm{H}_{2} \mathrm{O} & (0.0012 \underline{\mathrm{M}}) * \\ 21 & " & 1.30 & " & \mathrm{NaHCO}_{3} " & (0.0248 \underline{\mathrm{M}}) *\end{array}$

*Final concentration

Ionic strength $=0.16$

$$
\left(\mathrm{Ca}^{+}+=11.5 \mathrm{mg} / 100 \mathrm{ml}\right)
$$


Twenty milligrams of each of the amino acids of calcitonin except the glycine-C-14 (UL) and 3.2 grams of glucose were added to each liter of freshly prepared buffer. A 95\% oxygen-5\% carbon dioxide mixture was bubbled into the medium for 15 minutes and the ph was adjusted to 7.4 before it was used for the incubation.

The calcium ion concentration of the above mentioned KrebsRinger bicarbonate buffer was altered to prepare-incubation media containing low calcium $\left(4.8 \mathrm{mg} \mathrm{Ca}^{++} / 100 \mathrm{ml}\right)$ and high calcium $\left(26.0 \mathrm{mg} \mathrm{Ca}{ }^{++} / 100 \mathrm{ml}\right)$ concentrations. The composition of media is represented as follows:

Low-calcium medium

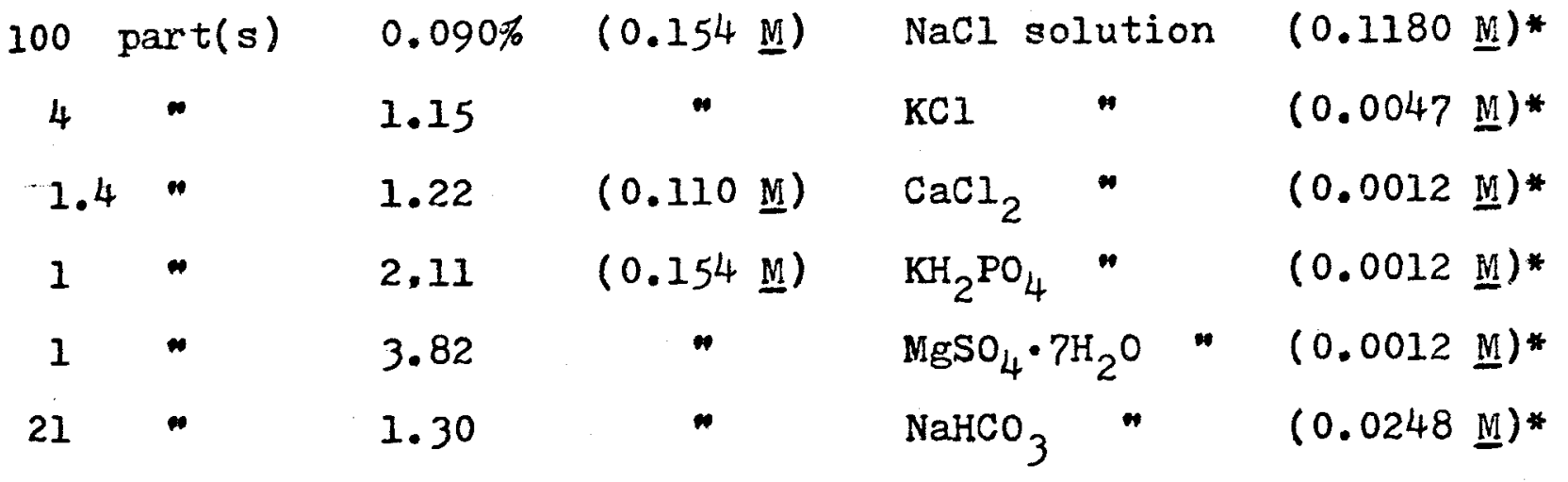

* Final concentration

Ionic strength $=0.16$

$$
\left(\mathrm{Ca}^{++}=4.8 \mathrm{mg} / 100 \mathrm{ml}\right)
$$


High-calcium medium

\begin{tabular}{|c|c|c|c|c|c|c|c|}
\hline 100 & $\operatorname{part}(\mathrm{s})$ & $0.90 \%$ & $(0.154 \mathrm{M})$ & $\mathrm{NaCl}$ & luti & on & $(0.1180 \mathrm{M})^{*}$ \\
\hline 4 & " & 1.15 & " & $\mathrm{KCl}$ & $"$ & & $(0.0047 \mathrm{M}) *$ \\
\hline 8 & $\omega$ & 1.22 & $(0.110 \mathrm{M})$ & $\mathrm{CaCl}_{2}$ & $"$ & & $(0.0062 \mathrm{M}) *$ \\
\hline 1 & $\infty$ & 2.11 & $(0.154 \mathrm{M})$ & $\mathrm{KH}_{2} \mathrm{PO}$ & $"$ & & $(0.0012 \underline{M}) *$ \\
\hline 1 & $"$ & 3.82 & $"$ & $\mathrm{MgSO}_{4}$ & & $"$ & $(0.0012 \mathrm{M}) *$ \\
\hline 21 & 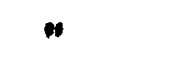 & 1.30 & $n$ & $\mathrm{NaHCO}$ & & & $(0.0248 \mathrm{M}) *$ \\
\hline
\end{tabular}

* Final concentration

Ionic strength $=0.17$

$$
\left(\mathrm{Ca}^{++}=26.0 \mathrm{mg} / 100 \mathrm{ml}\right)
$$

\section{TISSUE PREPARATION AND INCUBATION}

Ovine thyroid glands were obtained fresh from the slaughterhouse, usually 10 to 20 minutes after the sheep were killed. The glands with all adhering tissues were placed in cold Krebs-Ringer bicarbonate buffer ( $\mathrm{pH} 7.4$ ) containing $320 \mathrm{mg}$ glucose/100 ml, and $20 \mathrm{mg} / \mathrm{L}$ of all the amino acids of calcitonin. The glands were brought to the laboratory where they were dissected free from fat, connective tissue, and possible contaminating parathyroid tissue. In sheep, one of the two pairs of parathyroid glands is located near the posterior extremity on the deep surface of the lobes of the thyroid. The whole thyroid glands were then sliced in the cold into slices approximately $0.5-1.0 \mathrm{~mm}$ in thickness with a StadieRiggs tissue slicer. The time interval between killing of the 
sheep and incubation of about 100 grams of thyroid slices was usually three hours. Approximately two grams of slices were placed in each of a series of $50 \mathrm{ml}$ Erlenmeyer flasks. Each flask contained $10 \mathrm{ml}$ of Krebs-Ringer bicarbonate buffer and $2.5 \mu \mathrm{Ci}$ of the labeled glycine. Air served as the gas phase and all incubations were carried out in a hood for 4 hours at $37^{\circ} \mathrm{C}$ in a shaker water bath. The incubation period was terminated by placing the Erlenmeyer flasks containing the thyroid slices into an ice bath.

To determine the effect of low, physiological, and high calcium ion concentration on altering the hypocalcemic response (a reflection of the synthesis of calcitonin) of the mitochondrial, nuclear, and microsomal preparations, the incubations were carried out in buffer containing low calcium $\left(4.8 \mathrm{mg} \mathrm{Ca}^{++} / 100 \mathrm{ml}\right)$, physiological calcium $\left(11.5 \mathrm{mg} \mathrm{Ca}^{++} / 100 \mathrm{ml}\right)$, and high calcium $\left(26.0 \mathrm{mg} \mathrm{Ca}^{++} /\right.$ $100 \mathrm{ml}$ ) concentrations. After the 4 hour incubation period, the thyroid slices were thoroughly homogenized and mitochondrial, nuclear, and microsomal subcellular fractions were obtained by differential centrifugation.

\section{ISOLATION SUBCELLULAR FRACTIONS}

After the $4 \mathrm{hr}$ incubation period, the ovine thyroid slices were minced with scissors. Mitochondrial, nuclear, and microsomal subcellular fractions were obtained by utilizing a fractionation scheme described by Mahler and Cordes (1966) in their text, Biological Chemistry. A $25 \%$ thyroid homogenate was prepared by placing $5 \mathrm{gm}$ of minced thyroid tissue in $15 \mathrm{ml}$ of "homogenizing 
medium" ( $0.32 \mathrm{M}$ sucrose, containing $0.02 \mathrm{M} \mathrm{Tris,} \mathrm{pH} 7.6$, and $3 \mathrm{x}$ $10^{-3} \mathrm{M} \mathrm{MgCl}_{2}$ ) in a glass Duall homogenizer. Five slow, up and down pestle movements were used to break up the fibrous tissue which is dispersed throughout the thyroid gland. This crude homogenate was then filtered through 2 layers of cheesecloth to remove the remnants of the fibrous tissue. The filtrate was then transferred to a Kontes Teflon homogenizer for a more thorough homogenization ( 15 slow, up and down pestle movements) to completely disrupt cell walls. Each $12.5 \mathrm{ml}$ of homogenized thyroid preparation was diluted to a final volume of $20 \mathrm{ml}$ by adding $7.5 \mathrm{ml}$ of "diluting medium" $(0.25 \mathrm{M}$ sucrose containing $0.02 \mathrm{M}$ Tris, $\mathrm{pH} 7.6$, and 3 $\left.\times 10^{-3} \mathrm{M} \mathrm{MgCl}_{2}\right)$. Twenty milliliters of homogenizing medium was then layered underneath this preparation. The entire volume was centrifuged for 10 minutes at $700 \mathrm{x} \mathrm{g}$ at $4^{\circ} \mathrm{C}$ in an International Model PR-2 Centrifuge (International Equipment, Company, Needham Hts., Massachusetts). Two fractions were obtained: (1) a supernatant fraction, containing mitochondrial and microsomal material, and (2) a residue, termed the crude nuclear preparation. The residue was resuspended in $13 \mathrm{ml}$ of a solution of $2.4 \mathrm{M}$ sucrose containing 1.0 $\mathrm{mM} \mathrm{MgCl}{ }_{2}$. The crude nuclear preparation was centrifuged for $1 \mathrm{hr}$ at $50,000 \times \mathrm{g}$ at $4^{\circ} \mathrm{C}$ in a Spinco Model I Ultracentrifuge (Beckman Instruments Incorporated, Palo Alto, California). Two fractions were obtained: (1) the supernatant which was discarded, and

a residue, termed the purified nuclei. The purified nuclear preparation was then taken up in $1 \mathrm{ml}$ of diluting medium. This preparation was checked for homogeneity by phase contrast and electron microscopy. The electron microscopy was performed by Dr. John U. Balis Associate Professor of Pathology, Loyola University, Medical Center. 
Twenty milliliters of 0.1 M formic acid was added to the suspension and the entire preparation was lyophilized.

The supernatant fraction containing mitochondrial and microsomal material was thoroughly mixed and centrifuged for 10 minutes at $7,000 \times \mathrm{g}$ at $4^{\circ} \mathrm{C}$ in the International Centrifuge resulting in a residue, termed the crude mitochondrial preparation, and a supernatant, containing crude microsomal material. The crude mitochondrial preparation was taken up in $10 \mathrm{ml}$ of diluting medium and centrifuged for 10 minutes at $24,000 \mathrm{x}$ at $4^{\circ} \mathrm{C}$ in the International Centrifuge. The residue from this centrifugation was termed the purified mitochondrial preparation and the identity of this preparation was established by examining an aliquot by phase contrast and electron microscopy. This fraction was prepared for lyophilization in a manner identical to the nuclear preparation. The supernatant, containing crude microsomes, was added to the $7,000 \times \mathrm{g}$ supernatant. This combined material was then centrifuged for 100 minutes at $105,000 \mathrm{x} \mathrm{g}$ at $4^{\circ} \mathrm{C}$ in the Spinco Ultracentrifuge. The supernatant was discarded. The residue, termed the crude microsomal preparation, comprised of free ribosomes and smooth and rough endoplasmic reticulum, and dense granules, was suspended in one $\mathrm{ml}$ of diluting medium. The identity of this subcellular fraction was checked by electron microscopy. The remainder of the crude microsomal preparation was added to $20 \mathrm{ml}$ of $0.1 \mathrm{M}$ formic acid and lyophilized. A diagram of the scheme for the subcellular fractionation is shown in Figure 3. Electron micrographs of the microsomal and mitochondrial fractions are shown in Figure 4 and in Figure 5, while a phase contrast photograph of the nuclear fraction is presented in Figure 6. 


\section{Subcellular Fractionation Scheme}

$25 \%$ Thyroid homogenate (1part tissue, 3 parts homogenizing medium). Prepared in a Duall homogenizer. 5 up and down pestle movements.

Teflon homogenizer-15 pestle movements \& filtered.<smiles>[AlH2]</smiles>

Diluted each $12.5 \mathrm{ml}$ of homogenized tissue to a final volume of $20 \mathrm{ml}$ using the diluting medium .

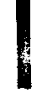

Layered $20 \mathrm{ml}$ of homogenizing medium underneath this preparation.<smiles>Ic1ccccc1</smiles>

- Centrifuged for 10 minutes at $700-\mathrm{g}$ at $4^{\circ} \mathrm{C}$.

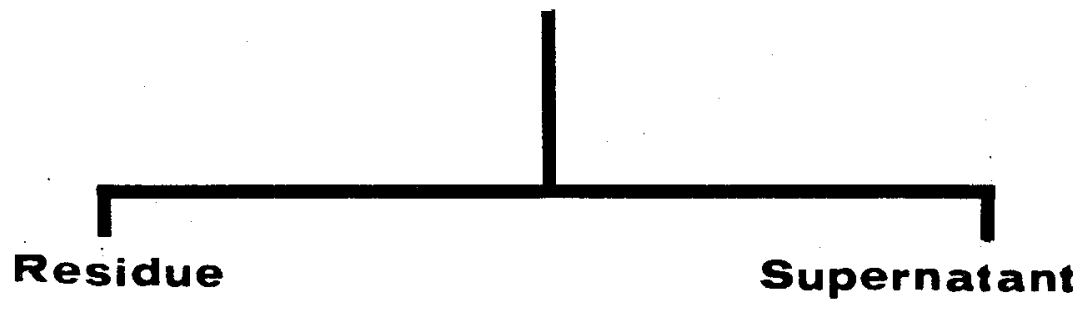

Figure 3. Fractionation scheme utilized to obtain mitochondrial, nuclear, \& microsomal tractions from incubated ovine thyroid gland slices. 
Residue (crude nuclei) resuspended

Supernatant - centrifuged for to a volume of $13 \mathrm{ml}$ in $2.4 \mathrm{M}$ sucrose.

Cenitrifuged 60 minutes at $50,000-9$

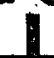

\section{Residue}

(purified nuclei)

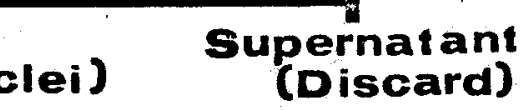

10 minutes at $7,000-g$

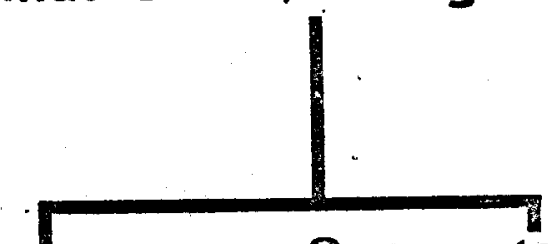

Supernatant Centrifuged 100 minutes at $105,000-g$

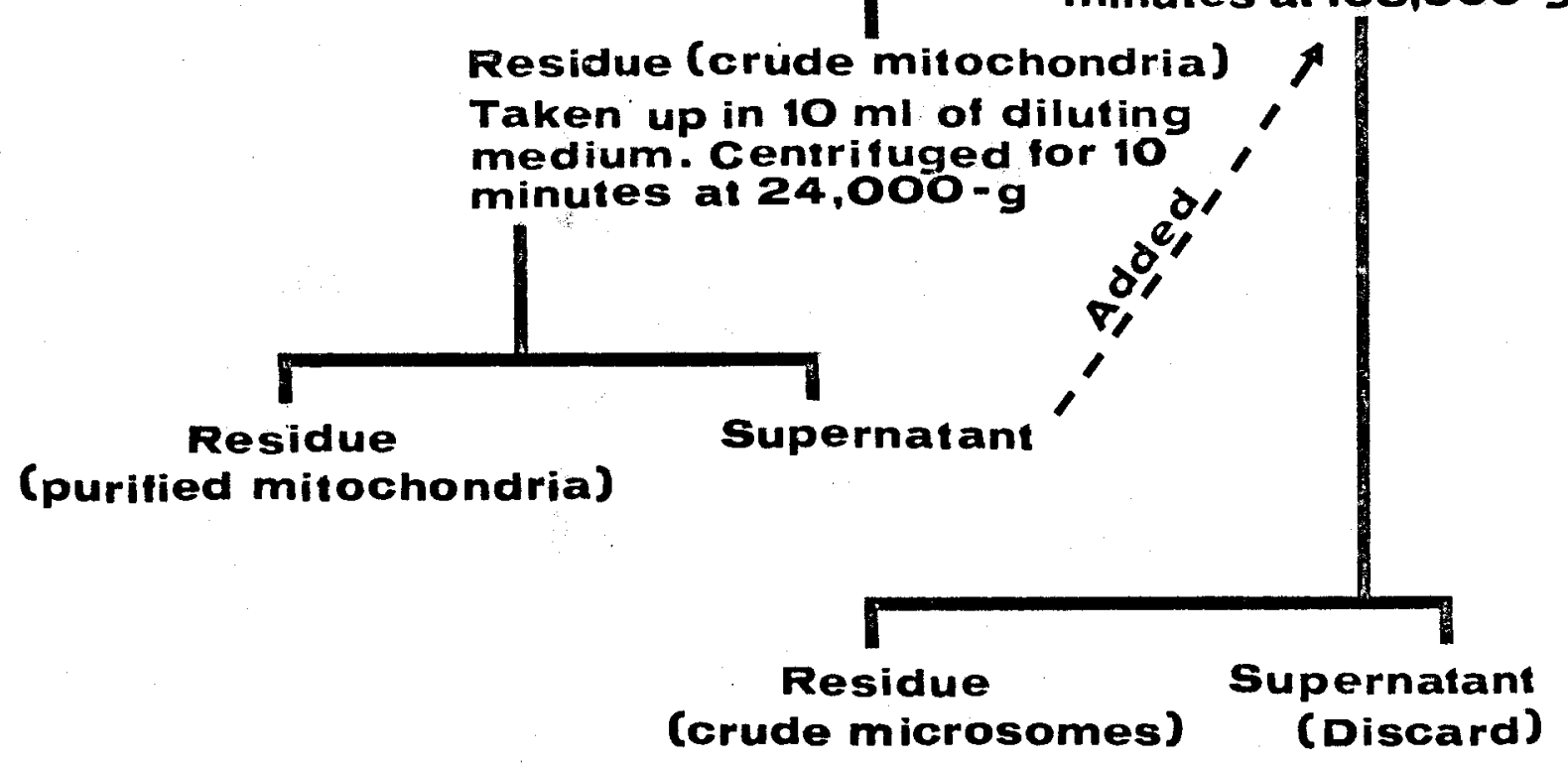




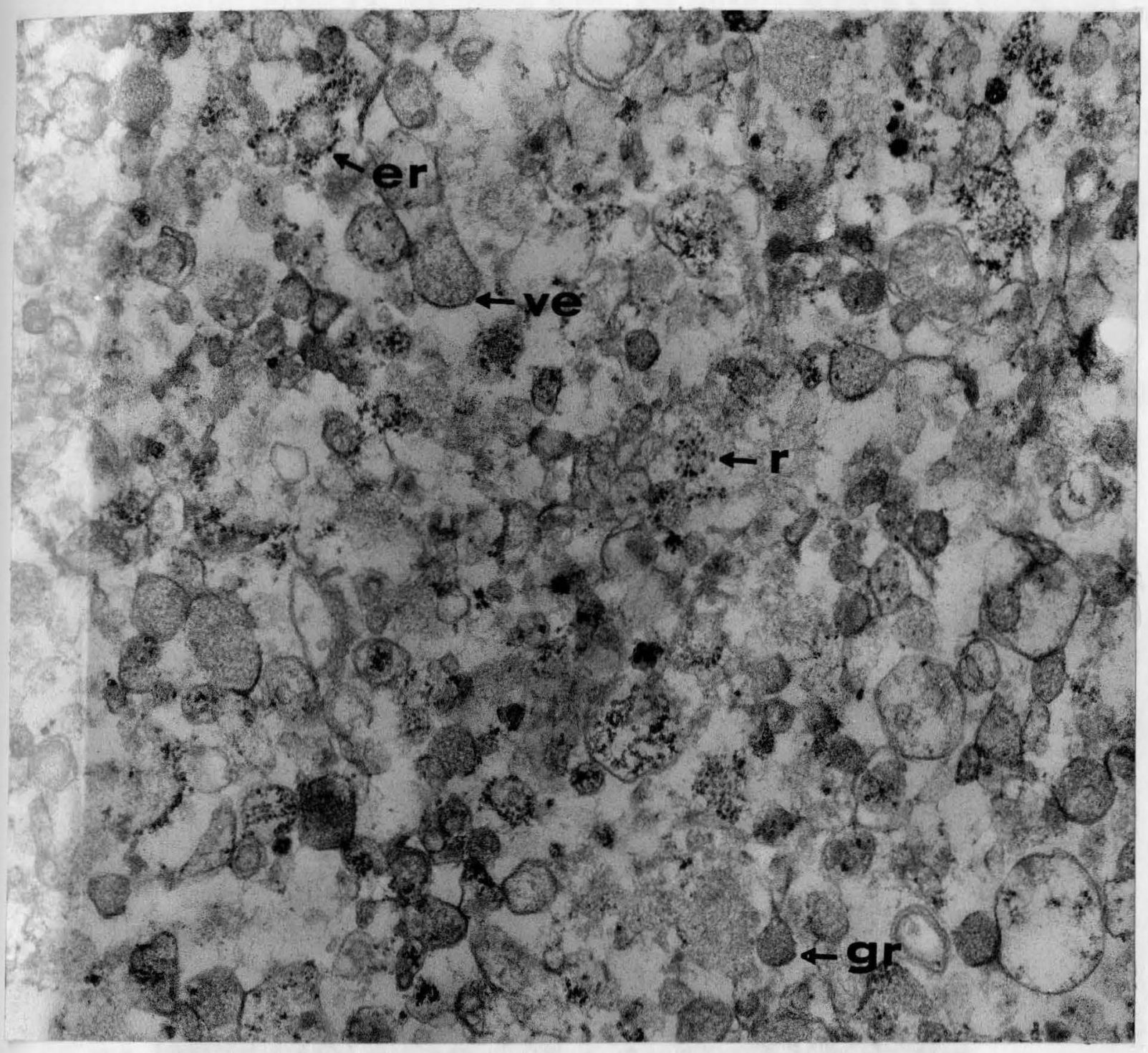

Figure 4. Electron micrograph of the thyroid microsomal fraction $(105,000 \mathrm{xg})$ containing smooth and rough endoplasmic reticulum, free ribosomes, and dense granules. Very little contamination from the mitochondrial fraction was observed $(x 39,500)$. 


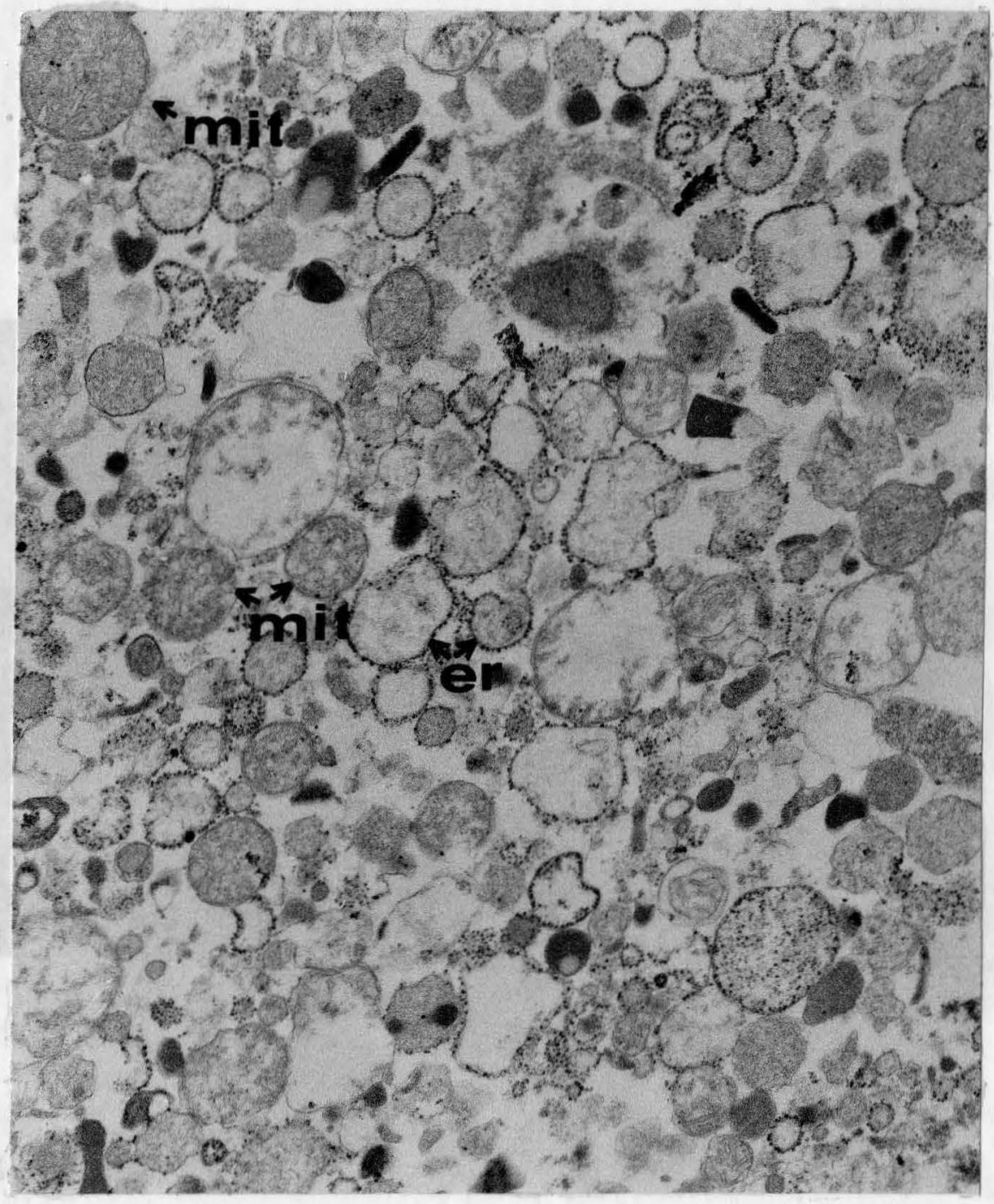

Figure 5. Electron micrograph of a crude mitochondrial fraction $(7,000 \mathrm{xg})$ exhibiting some microsomal contamination. Purified thyroid mitochondrial fractions $(24,000 \mathrm{xg})$ were assayed for hypocalcemic activity. 


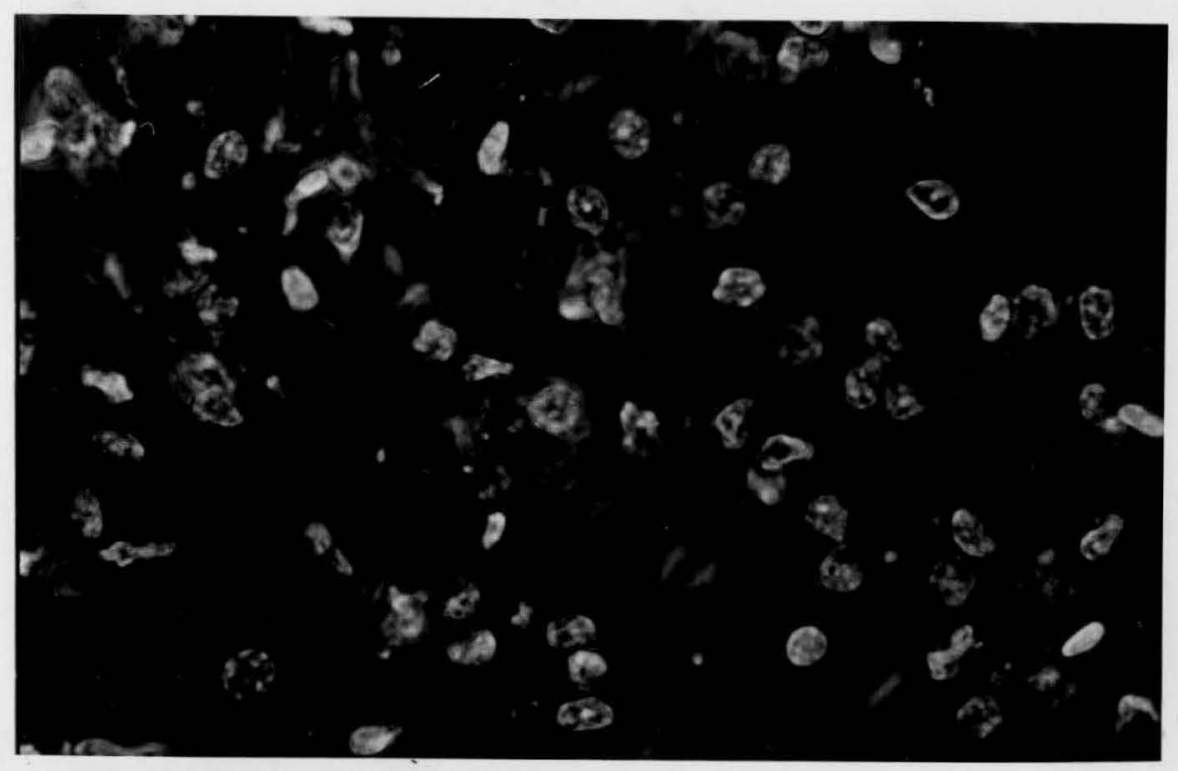

Figure 6. Phase contrast photograph of a purified thyroid nuclear fraction $(50,000 \mathrm{xg})$. Very little contamination was observed $(x 550)$. 
In some instances, the mitochondrial, nuclear, and microsomal preparations were assayed for hypocalcemic activity in rats without further purification. Two milligrams of the respective subcellular fractions were dissolved in $250 \mu \mathrm{I}$ of deionized water and injected intravenously into rats. Subcellular fractions were purified by dissolving the lyophilized preparations in 0.1 M formic acid $(18 \mathrm{mg} / \mathrm{ml})$ and applying the dissolved material to a $2.5 \times 60 \mathrm{~cm}$ column of Sephadex G-100 (Pharmacia Fine Chemicals, Inc., Picataway, New Jersey) and eluting with the same solvent. The transmission of the effluent was monitored with a Uvicord Absorptiometer and Recorder (LKB Instruments, Inc., Rockville, Maryland) and 50 drop fractions (approximately $4 \mathrm{ml}$ per fraction) were collected on a Unifrac Fraction Collector (Savant Instruments Inc., Hicksville, New York). The fractions representing the elution peak, usually numbering 6 to $?$ were pooled and lyophilized in $0.1 \mathrm{M}$ formic acid as were the 10 to 12 fractions beyond the elution peak. In every instance, lyophilized material could be recovered only from the pooled fractions representing the elution peak. These purified subcellular fractions were assayed for radioactivity, protein content, and hypocalcemic activity. In some instances, microsomal preparations which had already been passed through a Sephadex G-100 column were purified further by passage through a $2.5 \times 45 \mathrm{~cm}$ column (at a concentration of $2.5 \mathrm{mg} / \mathrm{ml}$ ) of Sephadex G-50 Superfine and eluted with $0.1 \mathrm{M}$ formic acid. The microsomal fractions obtained from the elution peak (usually numbering 4 to 5 ) were then lyophilized. Lyophilized microsomal material from the elution peaks of both Sephadex G-100 and G-50 columns were assayed for homo- 
geneity by polyacrylamide gel electrophoresis. The icientity of each of these microsomal preparations was established by fractionating a standard calcitonin preparation under identical conditions in polyacrylamide gels.

\section{SOLUTIONS}

Homogenizing Medium: $109.6 \mathrm{gm}$ of sucrose $\left(\mathrm{C}_{12} \mathrm{H}_{22} \mathrm{O}_{11}\right), 0.61 \mathrm{gm}$ of $\mathrm{MgCl}_{2} \cdot 6 \mathrm{H}_{2} \mathrm{O}$, and $1.7 \mathrm{gm}$ of $\operatorname{Trizma-HCl}$ (Tris (hydroxymethyl) aminomethane hydrochloride) and $1.4 \mathrm{gm}$ of Trisma-Base (Tris (hydroxymethyl) aminomethane) were dissolved in one liter of deionized water. A pH of 7.6 was attained by slight adjustments of either the Trisma-Base or Trisma-HCl concentrations.

Diluting Medium: $85.6 \mathrm{gm}$ of sucrose, $0.61 \mathrm{gm}$ of $\mathrm{MgCl}_{2} \cdot 6 \mathrm{H}_{2} 0,1.7 \mathrm{gm}$ of Trizma-HCl, and $1.4 \mathrm{gm}$ of Trizma-Base were dissolved in one liter of deionized water.

$2.4 \mathrm{M}$ Sucrose Solution: $822 \mathrm{gm}$ of sucrose and $0.2 \mathrm{gm}$ of $\mathrm{MgCl}_{2} \cdot 6 \mathrm{H}_{2} \mathrm{O}$ were dissolved in one liter of deionized water. 
DETERMINATION OF SERUM CALCIUM

Serum calcium was determined with the use of the HPE Calcium Determination KIT, commercially available from the American Monitor corporation, Indianapolis, Indiana. The technique is a modification of Burr's (1969) automated method of serum calcium detection. The Monitor HPE Calcium Determination utilizes a dye, di(o-hydroxyphenylimine)-ethane in methyl alcohol. This dye forms a colored complex with calcium at pH 12. Specificity for calcium is maintained by a combination of dye-specificity and pH. The dye binds preferentially to calcium, forming a red-colored complex which is spectrophotometrically determined at $545 \mathrm{~nm}$. Polyvinylpyrrollidone is utilized in this method to catalyze the complexing of phosphate by molybdate, thus eliminating occasional low results caused by the formation of a phosphate-calcium complex. Individual serum blanks are obtained by complexing all calcium. Calcium and phosphate complexes are quickly dissociated at an acid pH. The dissociated phosphate is removed by a catalyzed reaction with molybdate. A strongly alkaline $\mathrm{pH}$ is then established. The phosphate remains complexed with molybdate and calcium reacts with the dye causing a change from a pale yellow to an intense red-colored complex.

Blood samples $(400 \mu \mathrm{l})$ were collected in microcentrifuge tubes by tail bleeding. (cutting off $\frac{1}{4}$ inch of the tip of the tail) of anesthetized rats. Blood was obtained from each rat before and 50 minutes after the injection of aliquots of the three subcellular fractions. The blood was allowed to clot for 30 minutes before the sample was centrifuged for 4 minutes in a Beckman Spinco Microfuge resulting in approximately 200 ul of serum. Fifty microliters of 
serum collected from each rat before and 50 minutes after the injection of aliquots of the test material was utilized for the determination of serum calcium levels.

The calcium determination is carried out by the addition of $1 \mathrm{ml}$ of molybdic acid reagent to a plastic tube (glass tubes often leach out calcium and interfere with this sensitive assay) containing molybdate and polyvinylpyrrolidone. This tube is termed the "Reagent Blank" and the same amount of acid reagent is added to a second tube termed the "Test." One then adds $50 \mu 1$ of rat serum to the "Test" tube followed by a thorough mixing. This is followed by the addition of $2 \mathrm{ml}$ of $0.2 \mathrm{~N} \mathrm{NaOH}$ to both the "Reagent Blank" and the "Test" tubes followed by mixing of the contents. Two milliliters of $0.0002 \mathrm{M}$ di(o-hydroxyphenylimine)ethane are then added to both the "Reagent Blank" and the "Test" tubes and both are mixed once again. After waiting two minutes, one determines the absorbance of the "Test tube and the "Reagent Blank" at $545 \mathrm{~nm}$ with a Beckman DU Spectrophotometer. Readings are performed within 5 minutes of completion of the procedure since color stability decreases significantly after 10 minutes.

\section{Calculations}

$$
\text { Serum Calcium }(\mathrm{mg} \%)=\frac{\text { Absorbance of Unknown }}{\begin{array}{c}
\text { Absorbance of } 10.0 \mathrm{mg} \% \\
\text { Calcium Standard }
\end{array}} \times \begin{aligned}
& \text { Concentration } \\
& \text { of the } \\
& \text { Standard }(10.0 \mathrm{mg} \%)
\end{aligned}
$$


PROTEIN ANALYSIS

Frotein was determined by utilizing the method of Lowry (1951) as modified by Oyama and Eagle (1956). There are two distinct steps which lead to the final color with protein: (1) a reaction with copper in alkali, and (2) a reduction of the phosphomolybdicphosphotungstic acid reagent by the copper-treated protein. One milliliter of reagent " $C$ " was placed in each of a series of $10 \mathrm{x}$ $75 \mathrm{~mm}$ test tubes. Two hundred microliters of sample was added to the sample tubes and $200 \mu \mathrm{l}$ of deionized water was added to each blank tube. The contents of each tube were immediately mixed on a vortex mixer and allowed to stand for 10 minutes. Then $0.1 \mathrm{ml}$ of Folin-Ciocalteu (Hartman-Leddon Company, Philadelphia, Pa.) reagent was added to each tube and the samples were thoroughly mixed again. The reaction mixtures were allowed to stand undisturbed for a minimum of 30 minutes while color development of the reaction was completed. The transmittance of blank and sample solutions was determined with a Beckman DU spectrophotometer at a wavelength setting of $750 \mathrm{~nm}$. A standard curve was prepared following this procedure using bovine serum albumin as the standard. The calibration curve data are indicated in Table $I$ and the standard curve is shown in Figure 7 .

\section{Calculations}

The percent transmittance values are converted to absorbance and the density of the reagent blank is subtracted from that of unknown and standard preparations to obtain the absorbance of each 


\section{TABLE I}

STANDARD CURVE DATA FOR PROTEIN DETERMINATION

Albumin $(\mu \mathrm{g})$

Number of

Determinations

Absorbance

$750 \mathrm{~nm}$

5

6

0.0932

10

6

0.1435

20

5

0.2434

30

6

0.3229

40

5

0.3975

50

6

0.4690 


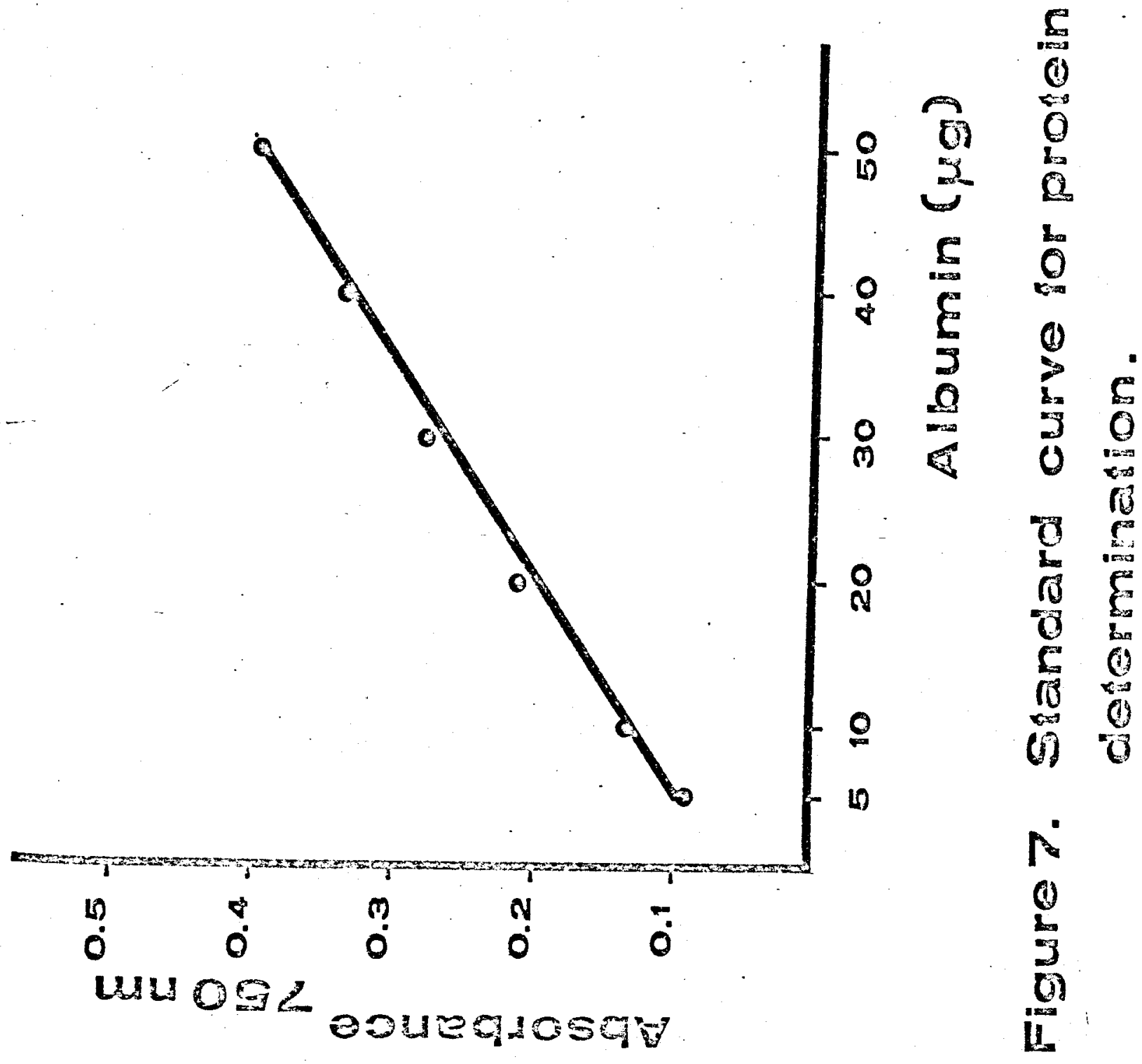


of these preparations. The concentration of the unknown ( $\mu \mathrm{g}$ protein/ ml of unknown sample) is calculated using the formula

$\mu g$ of protein/
mi of unknown $=\frac{\text { Absorbance of Unknown }}{\text { Absorbance of Standard }} \times \begin{aligned} & \text { (concentration of } \\ & \text { standard in } \mu g)\end{aligned} \times 5$

\section{Solutions}

Reagent A: $20 \mathrm{gm}$ of $\mathrm{Na}_{2} \mathrm{CO}_{3}, 4 \mathrm{gm}$ of $\mathrm{NaOH}$ and $200 \mathrm{mg}$ of $\mathrm{KNaC}_{4} \mathrm{H}_{4} \mathrm{O}_{6}$ $.4 \mathrm{H}_{2} \mathrm{O}$ were dissolved in one liter of deionized water.

Reagent B: $5 \mathrm{gm}$ of $\mathrm{CuSO}_{4} \cdot 5 \mathrm{H}_{2} \mathrm{O}$ were dissolved in one liter of deionized water.

Folin-Ciocalteu Reagent: Folin-Ciocalteu Reagent was obtained from Hartman-Leddon Company and diluted with deionized water in the ratio of 5 parts of the phenol reagent to 7 parts of deionized water as recommended by Oyama and Eagle (1956).

Reagent $C:$ Fifty parts of reagent $A$ and one part of reagent $B$ were mixed fresh just prior to use. 
MEASUR EMENT OF RADIOACTIVITY

A Beckman LS-250 Liquid Scintillation Spectrometer was utilized for all measurements of radioactivity. Samples were counted in 22 ml low potassium-40 content glass vials with tin-foil lined plastic screw caps. Ten milliliters of scintillation fluid was added to each vial. The scintillation fluid mixture was prepared fresh on the day of use by dissolving $4.0 \mathrm{gm}$ of PPO in 1 liter of toluene of scintillation grade. Twenty milliliters of Bio-Solv solubilizer BBS-3 (Beckman Instruments, Fullerton, California) was then added to each $100 \mathrm{ml}$ of this fluid. An appropriate amount of sample was then added to the $10 \mathrm{ml}$ of scintillation fluid in each vial and the sample was counted three times, each time for 10 minutes.

The Automatic Quench Compensation function (AQC) of the Beckman instrument was utilized, the gain was set at 500 and the external standard Isoset was set at 0.766 . The 2 sigma statistical counting error ranged from 3 to $7 \%$. Radioactivity was expressed as disintegrations per minute $(\mathrm{dpm})$ per $\mathrm{mg}$ of protein. Quenching was not a problem in the measurement of the radioactivity of the samples. This conclusion was based upon an evaluation of a quench correction curve of a plot relating \% efficiency versus external standard ratio, for a series of counting vials prepared by adding various amounts of protein ( 2 to $10 \mathrm{mgs}$ ) isolated from the mitochondrial fraction of thyroid homogenates, in $1 \mathrm{ml}$ of $0.1 \mathrm{~N}$ formic acid, to $10 \mathrm{mls}$ of the toluene-based fluor containing 0.025 microcuries of ${ }^{14} \mathrm{C}$-glycine. No quenching effect was found. 
DETERMINATION OF BIOLOGICAL ACTIVITY

Male Holtzman rats (Holtzman Company, Madison, Wisconsin) in the weight range of 110 to 120 grams were kept in the animal rooms and fed a standard rat diet until they reached a weight of 130 to 160 grams. The animals were fasted 18 hours prior to their use for determination of hypocalcemic activity of standard calcitonin preparations and of mitochondrial, nuclear, and microsomal subcellular preparations. The rats were anesthetized with sodium pentobarbital which was injected intraperitoneally at a dose of $5 \mathrm{mg}$ per $100 \mathrm{gm}$ of body weight. At this dose level, the rats remained anesthetized for approximately one hour and a half. At zero time, a tail-blood sample was collected after cutting off $\frac{1}{4}$ inch of the tip of the rat's tail. The femoral vein of the rat was exposed and $2000 \mu \mathrm{g}$ of the desired subcellular preparation dissolved in $250 \mu l$ of deionized water was injected into the vein over a 30 second period using a $250 \mu \mathrm{l}$ Hamilton syringe. The incision was then closed with wound clips. A second blood sample was collected into a microcentrifuge tube by snipping off an additional portion of the tail fifty minutes after the injection of the test material. Kumar et al. (1965) noted that the maximal hypocalcemic response after intravenous injection of calcitonin preparations occurred 30 to 60 minutes after injection. They therefore selected 50 minutes as the standard collection time for the second blood sample.

The blood was allowed to clot for $\frac{1}{2}$ hour. It was then centrifuged in a Beckman Spinco Microfuge for 5 minutes to separate the serum. Serum calcium level $(\mathrm{mg} / 100 \mathrm{ml})$ was determined for both the time zero and fifty minute samples. Hypocalcemic activity was 
expressed as the change in serum calcium between time 0 and 50 minutes per $10 \mu \mathrm{g}$ of protein of the test materials.

POLYACRYIANIDE GEL ELECTROPHORESIS OF MICROSOMAL AND STANDARD

\section{CALCITONIN PREPARATIONS}

Standard calcitonin preparations having a potency of 88 MRC Units and microsomal preparations which had been passed through a Sephadex G-100 or through both a G-100 and G-50 columns were subjected to polyacrylamide gel electrophoresis utilizing a Canalco -Model 66 Disc Electrophoresis Unit (Canal Industrial Corp., Rockville, Maryland). This was done to establish the identity of the hypocalcemic microsomal fraction with a calcitonin preparation of known potency. Microsomal and standard calcitonin preparations were fractionated into their component protein bands in anionic polyacrylamide gels. In some instances, calcitonin standard preparations were fractionated in cationic gels and under identical conditions of milliamperage per gel column ( $2.5 \mathrm{ma})$, electrophoretic separation time ( 1 hour and 20 minutes), and gel concentration ( $7.5 \%$ ).

Electrophoresis was performed in small gel columns consisting of three sections (Figure 8); (1) a large-pore anticonvection gel, termed the sample gel, into which the ionic mixture (microsomal and standard calcitonin preparations) was introduced; (2) a large-pore gel, termed the spacer or stacking gel, which has an identical chemical composition to that of the sample gel and in which the sample was electrophoretically concentrated; (3) a small-pore gel, termed 


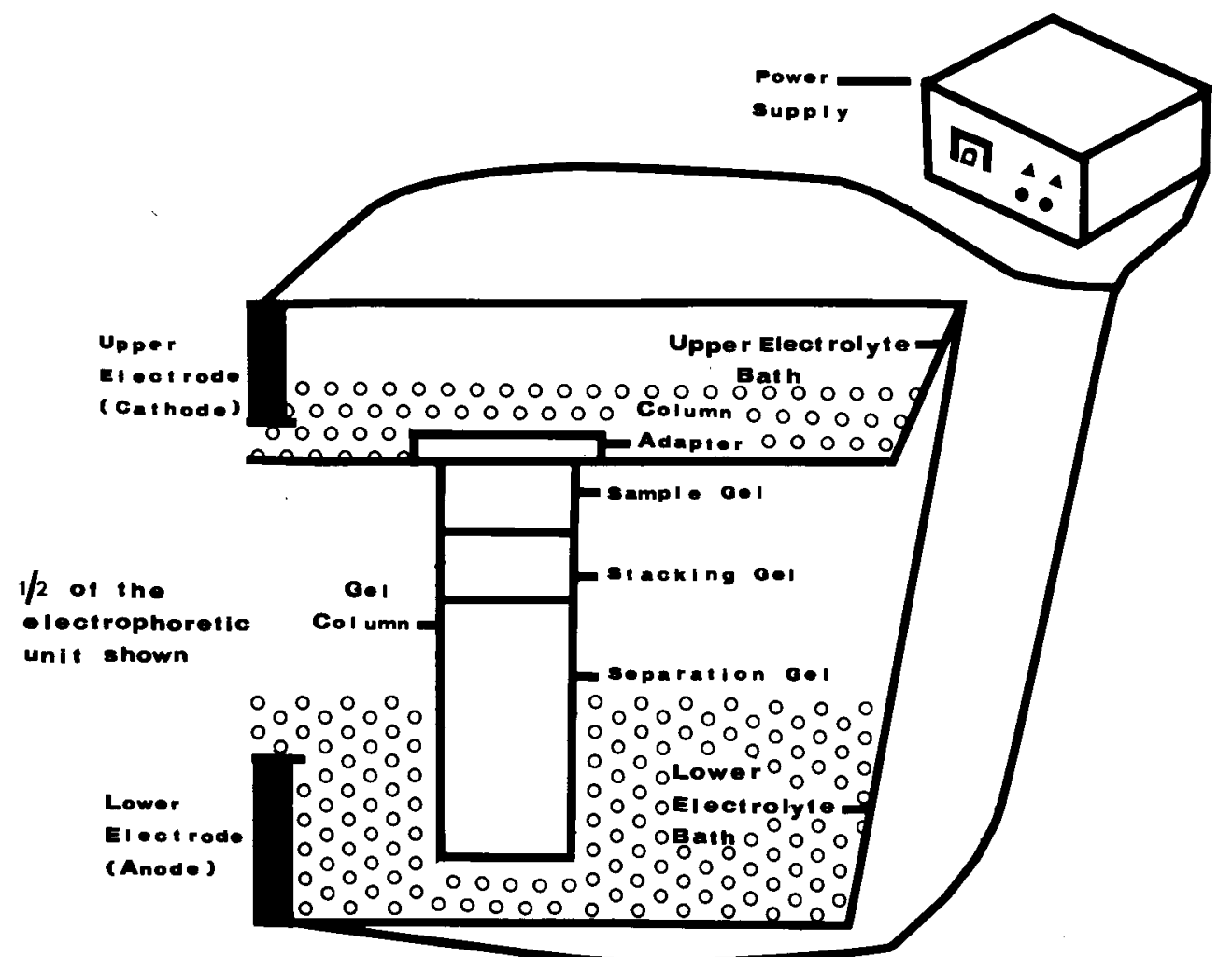

Figure 8. Disc electrophoresis is performed in glass columns filled with polyacrylamide gel consisting of a sample, stacking, and separation gel. A power supply, electrolyte compartments, and electrodes complete the arrangement. 
the running or separation gel, in which the sample was separated electrophoretically and by molecular sieving. The gel column was positioned between two buffer chambers and an electrical field was applied across tine gels. Each component migrated as a band with characteristic electrophoretic migration rate depending on its size and net charge.

Williams and Reisfeld (1964-65) have designated the necessary ionic constituents of a zonal polyacrylamide geI setup as the trailing ion (usually glycine or a weak acid or base), the leading ion (usually chloride ion), and the buffer counterion (Trizma-Base). The trailing ion (the correct term is trailing ion constituent, which -refers to a weak ion forming species regardless of whether or not it is actually ionized) is in two electrode compartments the leading ion is in the sample, spacer, and running gels; and the sample is in the sample gel. The buffer is in both the upper and lower electrode compartments as well as throughout the entire gel. A marked $\mathrm{pH}$ variation occurs at the boundary of the spacer gel and separation gel.

As the electrophoretic run commences (Figure 9), the sample Migrates electrophoretically from the sample gel into the stacking gel. In the stacking gel, two things happen to the migrating ionic species. First, the constituent ions of the sample are segregated from one another and stacked into contiguous zones. Their stacking alignment is dependent on the order of their relative electrophoretic mobilities. The entire sample is sandwiched between the trailand leading ions. Second, a concentration step for all these segregated stacks occurs. The thickness of these concentrated zones of 


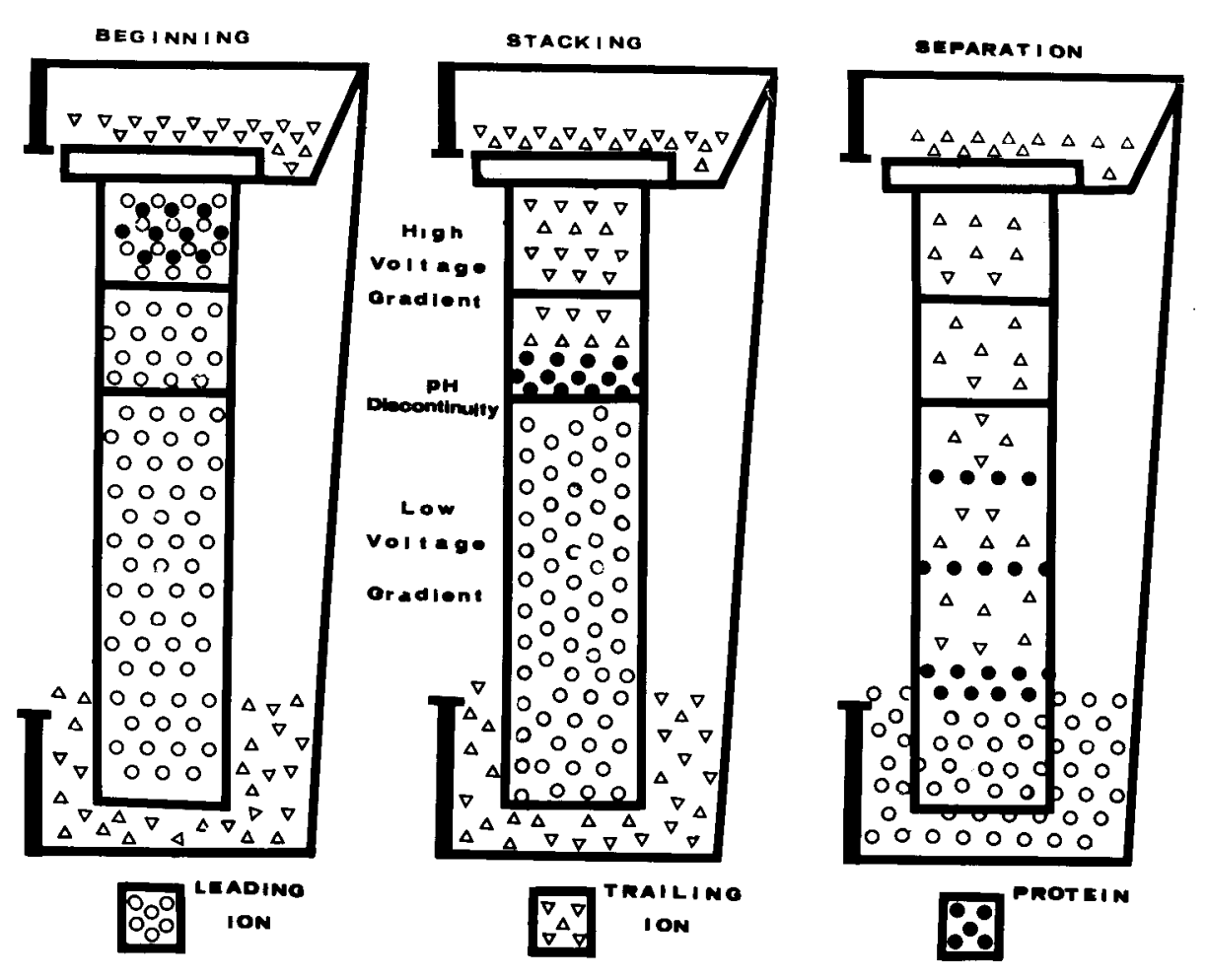

Figure 9. In polyacrylamide gel electrophoresis, the sample is first electrophoretically stacked. Once the components of this stacked zone have passed the $\mathrm{pH}$ discontinuity, they are separated electrophoretically and by molecular sieving. 
the various ionic species present in the stacks is independent of the initial concentrations in the mixture, but is entirely dependent upon the leading ion concentration. Therefore, sample mixtures which were initially very dilute in the sample gel can be readily stacked and concentrated in the stacking gel by varying the concentration of the leading ion whose movement in the gel sets up an electrical gradient. No molecular sieving effect is thought to occur in the sample and stacking gels. These are the two gel areas where electrophoretic effects predominate.

The concentrated sample ions in the stacking gel then migrate into the separation gel. At the stacking gel/separation gel interface a new set of conditions prevail. There is a discontinuity in $\mathrm{pH}$ between the gels and a change in pore size. As the leading ion/ trailing ion boundary passes the $\mathrm{pH}$ discontinuity of the stacking gel/separation gel interface, the previously observable schlieren boundary (boundary of leading ion/trailing ion) breaks up. It is at this stacking gel/separation gel interface that the mobility of the trailing ion now surpasses even that of the fastest sample ionic species. The trailing ion species which formerly had trailed the sample species in the stacking gel now continuously overtakes and passes through the sample species. This leading ion/trailing ion boundary migrates down the separation gel leaving behind a rather uniform voltage gradient in which the sample ionic species now resolve into a number of characteristic boundaries. This is due to the combined effects of the electrophoretic voltage gradient and the molecular sieving effect of the separation gel. The molecular sieving effect has been shown to depend upon the concentration of 
monomer used in the polymerization of the separation gel. Chrambach and Rodbard (1971) have shown that the synthetic polymer, polyacrylamide, can be made to provide an effective median pore radius of 0.5 to $3 \mathrm{~nm}$ by simply adjusting the total acrylamide concentration ( 3 to $30 \% \mathrm{w} / \mathrm{v}$ ), and the concentration of the cross-linking agent ( 1 to $25 \% \mathrm{w} / \mathrm{v}$ ) of the total monomer concentration in the polymerization reaction. The structural formula of acrylamide, the crosslinking reagent, initiators of the polymerization reaction, and the cross-linked polymer (polyacrylamide) are shown in Figure 10.

\section{STANDARD PROCEDURE--ANIONIC GEL SYSTEMS}

\section{Stock Solutions}

Stock solutions (TableII) were prepared using deionized water and were filtered and stored in brown glass bottles or bottles wrapped in foil in the refrigerator. Most of these stock solutions had a shelf-life of up to three months.

\section{Working Solutions}

Working solutions (Table III) were prepared fresh from the stock solutions on the day they were to be used. However, the persulfate solution, when kept stored in the refrigerator, was usable for a period of a week. 

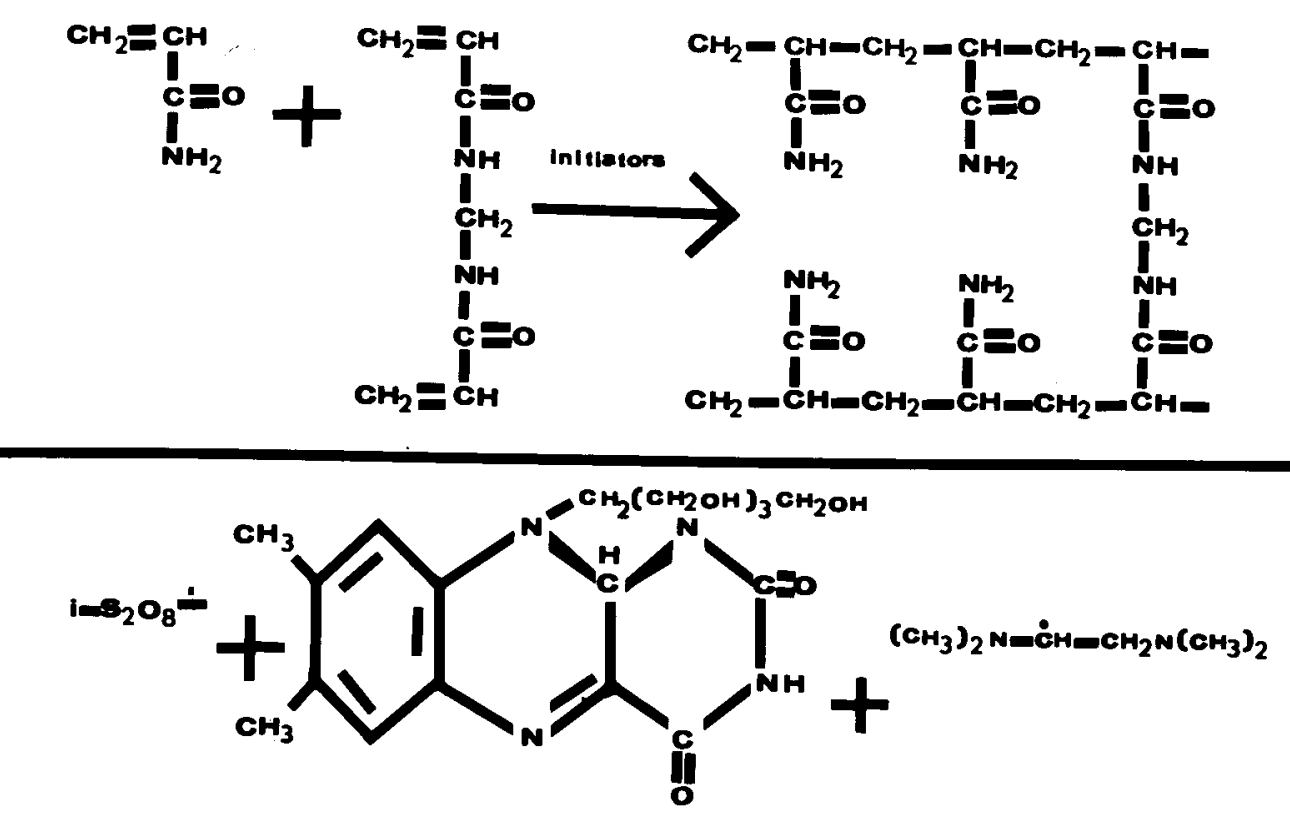

\section{+ LIGHT}

Figure 10. After Chrambach and Rodbard, Science (1971) 172 No. 3982. The polymerization reaction of acrylamide. The structures of acrylamide, $N, N^{\prime}$-methylenebisacrylamide and of a representative segment of cross-linked polyacrylamide are shown. Initiators shown are persulfate, riboflavin, and $\mathrm{N}, \mathrm{N}^{\prime} \mathrm{N}^{\prime}, \mathrm{N}^{\prime}$-tetramethylethylenediamine. 
TABLE II

ANIONIC GEL STOCK SOLUTIONS

Separation Gel

(Small-pore gel)
Sample and Stacking Gels

(Large-pore gels)

\section{(A)*}

1 N $\mathrm{HCl}$

TRIZMA-BASE

TEMED

water

to

\begin{abstract}
approx. $48 \mathrm{ml}$
\end{abstract} $36.3 \mathrm{gm}$

$0.46 \mathrm{ml}$

(pH 8.9)

$100 \mathrm{ml}$
(B)

$1 \underline{N} \mathrm{HCl}$

TRIZMA-BASE

approx. .48

$\mathrm{ml}$

TEMED

water

to

100

$5.98 \mathrm{gm}$

$0.46 \mathrm{ml}$

(pH 6.7)

(D)

Acrylamide

$10.0 \mathrm{gm}$

BIS

$2.5 \mathrm{gm}$

BIS

$0.8 \mathrm{gm}$

$0.015 \mathrm{gm}$

cyamide

water

to

100

$\mathrm{ml}$

water

to

100

$\mathrm{m}$.

(E)

(F)

Ammonium Persulfate

$0.14 \mathrm{gm}$

Riboflavin

$0.004 \mathrm{gm}$

water

to

$100 \mathrm{ml}$

water

to

100

$\mathrm{ml}$

* pH adjusted by titrating with I $\mathrm{N} \mathrm{HCl}$.

* Acrylamide is a neurotoxin in powder form--avoid inhaling.

The following abbreviations were used:

TEMED ( $N, N, N^{\prime}, N^{\prime}$-Tetrame thyle thylenediamine);

BIS $\left(N, N^{\prime}\right.$-Methylene-bisacrylamide). 


\section{TABLE III}

WORKING SOLUTIONS

separating Gel

(Small-pore gel)
Sample and Stacking Gel

(Large-pore gel)
1 Part A

2 Parts C

4 Parts E

1 Part Water

pH 8.9 (8.8-9.0)
1 Part B

2 Parts D

1 Part $F$

4 Parts Water

pH 6.7 (6.6-6.8)
Buffer Solution

TRIZMA-BASE $6.0 \mathrm{gm}$

Glycine $28.8 \mathrm{gm}$

Water to 1 liter

pH 8.3 
Eixative, Fixative-Stain, and Destaining Solutions

Fixative solutions ( $T a b l e I V$ ) and solutions for washing and destaining the polyacrylamide gels were prepared in advance and stored indefinitely at room temperature. The naphthol blue-black was added to acetic acid or trichloroacetic acid and the mixture was stirred and filtered through whatman No 1 filter paper. These solutions could be reused a number of times. The Coomassie Brilliant Blue $\mathrm{R}-250$ was prepared as an aqueous stock solution which was stirred and filtered prior to use. This stock solution was diluted 1:20 with trichloroacetic acid (TCA) just prior to staining the gels. The fixative-stain solution was made up fresh from the stock solution each time.

The following procedure was utilized to resolve microsomal and standard calcitonin preparations into their component protein fractions.

1) Pyrex glass columns $(6.5 \mathrm{~cm}$ long and having an inner diameter of $0.5 \mathrm{~cm}$ ) were acid cleaned and coated with a wetting agent ( 1 part Kodak Photo-Flo or Canalco Column-Coat to 200 parts water). Base caps (vacutainer tops) were placed at one end of the gel columns which were then placed into a loading rack in which front-to-back vertical alignment of the gels could be assured.

2) Stock solutions $A, C$, and $E$ were removed from the refrigera- 


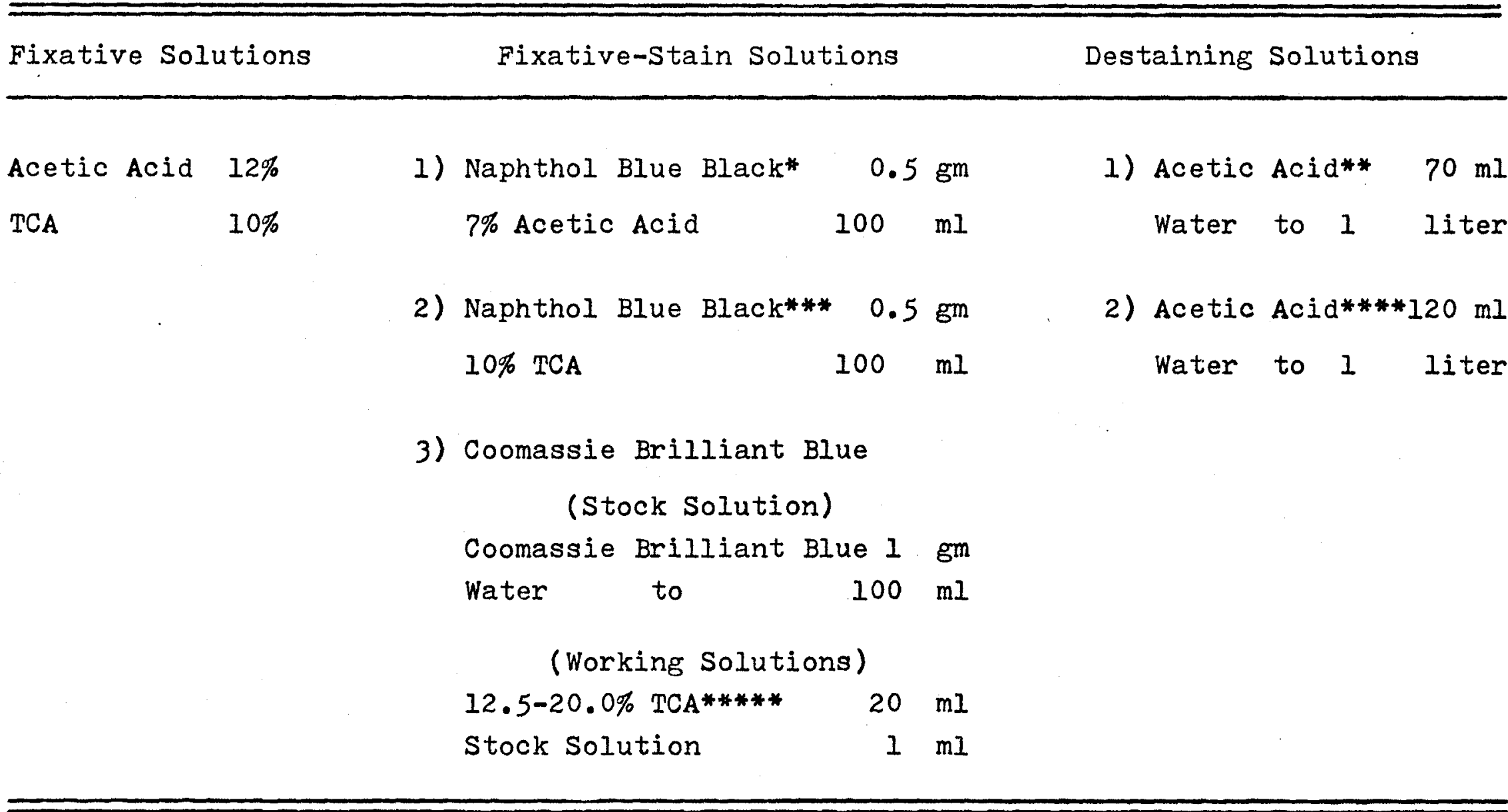

* Fixative-stain solution commonly used for serum proteins.

** Destaining solution commonly used for serum proteins.

* * Fixative-stain solution used for staining calcitonin.

*** Destaining solution used for calcitonin preparations.

**** Fixative-stain solution used for staining calcitonin preparations. A further increase in sensitivity can be achieved by the use of a $20 \%$ TCA dye solvent. 
tor and $2 \mathrm{ml}$ of solution $A, 8 \mathrm{ml}$ of solution $\mathrm{E}, 4 \mathrm{ml}$ of solution $\mathrm{C}$, and $2 \mathrm{ml}$ of deionized water were placed in separate flasks and allowed to warm up to room temperature. Sixteen mls of separation gel solution is the volume required to run twelve $6.5 \mathrm{~cm}$ long gels. When at room temperature, the four components were then added to a single flask and swirled gently to prevent air bubble formation and to provide thorough mixing. This was the separation gel solution. Using a $1 \mathrm{ml}$ tuberculin syringe, to which $5 \mathrm{~cm}$ of tygon tubing was attached, exactly $0.8 \mathrm{ml}$ of the separating gel solution was dispensed (without bubble formation) into each of the 12 vertical gel columns.

3) An Eppendorf or Oxford pipette was then used to carefully layer $100 \mu \mathrm{l}(2-3 \mathrm{~mm})$ of water on top of the gel solution. If the gel columns are not coated with a wetting agent prior to their use, a bolus of water will form which will drop into the gel solution and dilute it. However, if the gel columns are coated, water will layer evenly on top of the unpolymerized gel solutions. The purpose of the water layering of gel solutions is that it prevents a gel-air meniscus and produces a flat gel surface which is essential for obtaining good protein band formation. It also prevents oxygen, which retards gel formation, from diffusing into the gels. 
4) The separation gels remained undisturbed for one-half hour, during which time complete chemical polymerization occurred. one could determine if polymerization was complete by viewing the gel columns from the side. A distinct interface between the gel and water layers was visible when polymerization had occurred.

5) The gel columns were then removed from the loading rack and the water layer was removed by giving the column a quick flick of the wrist. The lip of the gel column was then touched to a piece of absorbant paper to remove the last traces of water.

6) Two milliliters of solution $B, 4 \mathrm{ml}$ of solution $D, 2 \mathrm{ml}$ of solution F, and $8 \mathrm{ml}$ of water were put into another set of small flasks. These solutions were warmed to room temperature and gently mixed in a flask that was completely wrapped in foil to protect this gel solution from premature photopolymerization. This solution was to serve as the photopolymerizing stacking and sample gel. A $1 \mathrm{ml}$ syringe with tygon tubing adapter was then used to add $0.2 \mathrm{ml}$ of this solution to each gel column. This was to serve as the stacking gel. Once again, $100 \mu l$ of water was layered over this gel solution as previously described. The entire loading rack was positioned approximately $30 \mathrm{~cm}$ from a fluorescent light source. This resulted in stacking gels with a more uniform pore size. Fifteen minutes were allowed for photopolymeriza- 
tion to begin as evidenced by the appearance of opalescence in the stacking gel. When photopolymerization had begun, the light source was moved to within $5 \mathrm{~cm}$ of the columns and another 15 minutes were allotted to insure total photopolymerization. The water layer was then removed as previously described.

\section{7) Sample Application}

Generally 200 to $500 \mu \mathrm{g}$ of solid sample can be resolved in a single polyacrylamide gel. However, samples that are relatively homogeneous can be assayed in quantities as small as 50 to $100 \mu \mathrm{g}$ range. Samples which are heterogeneous can be assayed at concentration ranges of 500 to $2,000 \mu \mathrm{g}$. Normally, calcitonin standard and microsomal preparations were dissolved in sufficient sample gel solution that $333 \mu \mathrm{g}$ of calcitonin standard and $1,500 \mu \mathrm{g}$ of microsomal calcitonin preparation were present in $0.2 \mathrm{ml}$ of sample gel solution. The sample gel solution $(0.2 \mathrm{ml})$ was added on top of the stacking gel and layered with $100 \mu I$ of water. The sample gel solution was allowed to photopolymerize for 30 minutes while positioned $5 \mathrm{~cm}$ from a fluorescent light source.

8) Bromphenol blue ( 0.1 to $0.2 \mathrm{ml})$, serving as a tracking dye, was incorporated into the sample gel solution prior to its addition to the gel column. This dye migrates faster than the fastest sample ion species. 
9) The gel columns were then removed from the loading rack and inserted into the holes of the upper bath stoppers (sample gel uppermost). After all the gel columns were inserted in this manner, buffer solution was added on top of the sample gels making certain that no air bubbles were entrapped in the remaining space of the upper gel column. The entire upper bath assembly containing the gel columns was then lifted out and sufficient buffer solution was added to the separation gels such that a hanging drop of buffer solution remained at the bottom of all the gel columns.

10) The lower bath (containing the anode) of the Canalco Electrophoresis unit was then filled with $500 \mathrm{ml}$ of buffer solution. The upper bath assembly was then carefully placed into position making certain that no air bubbles had formed at either end of the gel column. Air bubbles result in uneven band formation because a uniform current flow through the gels is prevented. The upper buffer reservoir of the Canalco apparatus was then filled with $250 \mathrm{ml}$ of buffer solution.

11) The upper bath lid was then placed on the upper bath. The power supply was connected. A current of 2.5 milliamps per gel column provided for good resolution of protein bands. Currents over 5 milliamps were avoided because they cause excessive ohmic heating within the gel, resulting in band distortion. Electrophoresis was carried out until the now discrete front of tracking dye band had migrated to within $\frac{3}{4}$ 
inch of the separation gel end. This required approximately 1 hour and 20 minutes at 2.5 ma per gel column.

12) At the completion of electrophoresis, the power supply was turned off and the electrode jacks were disconnected. Buffer from the upper and lower baths was decanted into separate containers and was reused a number of times in the respective buffer compartments as long as the upper buffer did not drop below $\mathrm{pH} 8.2$ or become contaminated with sample.

13) The gel columns were removed from the upper reservoir and were placed in a wash tray containing ice-cold water. The gels remained immersed in the ice-cold water for 5 minutes which was sufficient time for maximal gel contraction. A ten milliliter syringe fitted with an 18 gauge needle was filled with the ice-cold water. The gel columns were kept submerged while the tip of this needle (beveled edge towards the gel to avoid scratching the gel) was inserted between the glass column and the gel. The needle was inserted slightly beyond the sample gel of the gel column. Keeping the gel columns submerged and the needle flat against the glass surface, the entire gel column was rotated with one hand while the position of the needle was maintained. This freed the entire circumference of the sample gel from the glass column. Ice-cold water was continuously injected around the gel surface as the gel column was rotated. The water pressure freed the stacking and separating gels and in a few moments the intact gel was expelled 
from the separating gel end of the glass column.

14) Microsomal and calcitonin standard preparations were fixed and stained in fixative-stain No 2 (Table IV). Ten per cent TCA solution was found to be a good fixative for calcitonin preparations. The gels were fixed and stained in this solution for at least one hour. Another method that was used involved fixing the gels in 10\% TCA or $12 \%$ acetic acid for one hour. The gels were then placed in fixative-stain solution No 3 (Table IV) for a minimum of one hour or as long as 24 hours. Chrambach et al. (1967) demonstrated that Coomassie Brilliant Blue, the stain in fixative-stain No 3, was approximately five times as sensitive a stain for proteins and peptides when compared to the naphthol blue black stain. Minute protein bands were well detailed in 20\% TCA-Coomassie Brilliant Blue stain solutions. Gels that were fixed and stained with the No 3 fixative-stain solution destained themselves over a period of several weeks. Electrophoretic destaining of TCAfixed gels was more prolonged then those prepared in fixativestain solution No 1 .

15) Electrophoretic destaining of microsomal and standard calcitonin gels stained by fixative-stain No 2 (TableIV) was performed in the same apparatus. The gels, sample gel uppermost, were placed in destaining tubes, which had been attached by their wide ends to the upper buffer reservoir. The gels were wedged firmly against the constricted ends of the tubes. 
Mhe destaining tube was then carefully filled (using a syringe to avoid bubble formation) with destaining solution No 2. Five-hundred milliliters of destaining solution No 2 were poured into the lower bath of the Canalco apparatus. The upper reservoir was filled with $250 \mathrm{ml}$ of destaining solution No 2. The electrodes were connected, the cathode to the upper reservoir. A constant current of 4 ma per tube was set on the power supply. On applying the current, the unbound dye migrated down the gels into the lower reservoir. At this milliamperage setting, it took 2 to 3 hours to destain the gels.

16) When the section of gel containing no separated fractions was completely clear of stain, the power supply was turned off. The destaining solution was decanted, and the gels were transferred to small test tubes containing destaining solution No 2 for storage. Alternatively, the gels were stored in $10 \% \mathrm{TCA}$ or in a solution comprised of equal parts of glycerol and deionized water. This solution allows for storing the gels up to two years without shrinkage.

\section{STANDARD PROCEDURE--CATIONIC GEL SYSTEM}

In some instances, cationic gels were utilized to fractionate calcitonin standard preparations. 
Stock Solutions

Stock solutions (Table V) were prepared and stored in the same manner as anionic gel solutions.

Working Solutions

Working solutions (Table VI) were prepared fresh from the stock solutions on the day they were to be used.

Fixative and Fixative-Stain Solutions

Cationic gels were fixed in $10 \%$ TCA. Either fixative-stain solution No 2 or No 3 (see Table IV) were utilized for staining cationic gels. However, Coomassie Brilliant Blue in 12.5 to $20 \%$ TCA was the most sensitive and therefore the most commonly employed fixative-stain solution for cationic gels.

\section{Procedure}

The experimental procedure for running cationic gels was nearly identical to that described previously for anionic gels. Any differences in procedure are listed below:

1) Separation gels were allowed to stand undisturbed for one hour while chemical polymerization was completed (see step 4, anionic gel system).

2) Since electrical polarity had to be switched (see step 11, anionic gel system), no tracking dye (see step 8, anionic gel 
TABLE V

CATIONIC GEL STOCK SOLUTIONS

Separation Gel

Sample and Stacking Gels
(A)*
(B)*

$1 \underline{N} \mathrm{KOH}$

12

$\mathrm{ml}$

1 N KOH

24

$\mathrm{ml}$

Acetic Acid

53.2 $\mathrm{ml}$

Acetic Acid

$1.47 \mathrm{ml}$

TEMED

$0.24 \mathrm{mI}$

TEMED

$0.1 \mathrm{ml}$

Water to

100

$\mathrm{mI}$

Water to

100

$\mathrm{ml}$

(pH 2.9)

(pH 6.7)

(c)

(D)

Acrylamide

30

$g m$

Acrylamide

10

gm

BIS

$0.8 \mathrm{gm}$

BIS

0.8

gm

Water to

100

ml

Water to

100

ml

(E)

(F)

Ammonium Persulfate

$0.12 \mathrm{gm}$

Riboflavin

Water to

$0.002 \mathrm{gm}$

$\mathrm{ml}$

100

Ammonium Persulfate

Riboflavin

0.06

gm

Water to

$0.001 \mathrm{gm}$ $\mathrm{ml}$

* pH adjusted by titration with acetic acid. 
TABLE VI

WORKING SOLUTIONS

separating Gel Sample and Stacking Gel Buffer Solutions

\begin{tabular}{llclll}
2 Parts & A & 1 Part & B & \multicolumn{2}{c}{ UPPER BUFFER } \\
$1-1 \frac{1}{2}$ Parts* & C & $1-2$ Parts** & D & Glycine & 28.1 gm \\
1 Part** & E & 2 Parts & F & Acetic Acid & $3.05 \mathrm{ml}$ \\
$(\mathrm{pH} \mathrm{2.9)}$ & $(\mathrm{pH} \mathrm{6.7)}$ & Water to 1 liter
\end{tabular}

IOWER BUFFER

Acetic Acid $43 \mathrm{ml}$

1 N KOH $\quad 120 \quad \mathrm{ml}$

Water to 1 liter

$(\mathrm{pH}$ 4.3)

* Separating gel concentration may be increased in some instances to resolve protein samples into narrower bands (requires longer running time).

* Sample gel contration may be increased for samples which retard photopolymerization or those which are partially insoluble in sample gel solution.

** To insure stock solution stability, the catalyst, ammonium persulfate, may be deleted from stock solutions ( $E$ ) and (F) until the working solutions are being prepared. At that time, ammonium persulfate $(6 \mathrm{mg} / 5 \mathrm{ml})$ of stock solution $(E)$ and $3 \mathrm{mg} / 5 \mathrm{ml}$ of stock solution ( $F$ ) may be added to the proper ratios of stock solutions for the separation, stacking, and sample gels, pH of the buffer solutions if titrated with acetic acid. 
system) could be utilized to gauge the distance of electrophoretic migration of the standard calcitonin preparations. Therefore, one must experiment with different fractionation times (at constant milliamperage) to determine the length of electrophoresis that will result in optimal resolution of protein bands.

3) The lower bath (containing the cathode) was then filled with $500 \mathrm{ml}$ of the $1 \underline{\mathrm{N}} \mathrm{KOH} / \mathrm{acetic}$ acid buffer (see Table VI) at $\mathrm{pH} 4.3$ and the upper bath was filled with $250 \mathrm{ml}$ of glycine/ acetic acid buffer (see TableVI) at $\mathrm{pH} 4.0$.

\section{DENSITOMETRY OF POI.YACRYLAMIDE GELS}

Densitometry of gels was performed with a Densicord Model 542 densitometer (Photovolt Corporation, New York, N. Y.). This densitometer in conjunction with an automatic integrator attachment can be utilized to give accurate quantitation of all electrophoretically destained polyacrylamide gels. The proper response setting was chosen and the stained separation gels were scanned over their entire length by the Densicord. The automatic integrator attachment allows one to determine the percentages of the various fractions from the densitometer trace. The number of integrator marks was in exact proportion to the area under the curve at all points. 
PHOTOGRAPHY OF POLYACRYLAMIDE GELS

A Polaroid camera with a close-up lens was the most convenient method of photographing stained polyacrylamide gels.

\section{CHEMICALS REQUIRED FOR POIYACRYIAMIDE GEL ELECTROPHORFSIS}

Acrylamide, Eastman Organic Chemicals, No. 5521.

Bromphenol Blue (Water Soluble), Hartman-Ieddon Company, No. 859. Coomassie Brilliant Blue R250, Colab Laboratories, Inc., No. 11-152B. Glycine (Ammonia-free), Eastman Organic Chemicals, No. 445. $\mathrm{N}, \mathrm{N}^{\cdot-M e t h y l e n e-b i s ~ a c r y l a m i d e ~(B I S), ~ E a s t m a n ~ O r g a n i c ~ C h e m i c a l s, ~}$ No. $P 8383$.

Naphthol Blue Black, Eastman Organic Chemicals, No. 9763. Potassium Ferricyanide, Sargent-Welch Scientific Company, AR. 14180. Riboflavin, Eastman Organic Chemicals, No. 5181.

$N, N, N^{\circ}, N^{\circ}-$ Tetramethyethylene diamine (TEMED), Eastman Organic Chemicals, No. 8178. 
ISOETECTRIC FOCUSING

In recent years, the development of the highly sensitive isoelectric focusing technique has become a powerful tool for the analytical or preparative separation of ampholytes, especially proteins, and for the characterization of the ampholyte by determining its isoelectric point ( $\mathrm{pI}$ ). Isoelectric point determinations of resolved proteins can now be accomplished conveniently and with great reproducibility in a single experiment. The isoelectric focusing technique permits essentially complete analytical resolution of mixed protein components employing rather simply designed electrofocusing equipment and experimental protocol. One can now conveniently resolve protein components in a mixture which have as small a difference as $0.02 \mathrm{pH}$ units separating their isoelectric points.

Isoelectric focusing of proteins is achieved when a direct current potential is applied to a system of electrolytes in which the pH steadily increases from the anode to the cathode. Each protein species will be resolved in a stabilized $\mathrm{pH}$ gradient at the zone in the gradient at which the $\mathrm{pH}$ of the gradient corresponds to the pI of the species. The focusing, or resolution of proteins, is therefore attributable to the electric field they are in; hence, the name, isoelectric focusing. A necessary prerequisite is that the electrolyte system is stabilized against uncontrolled convection and against remixing of resolved proteins. This is accomplished by applying a linear sucrose gradient within the electrofocusing chamber of the column. 
Basic Principle

In isoelectric focusing of proteins, a pH gradient having the lowest $\mathrm{pH}$ values at the anode and the highest $\mathrm{pH}$ values at the cathode is utilized. When one adds a mixture of proteins with isoelectric points occurring within the $\mathrm{pH}$ range of the pH-gradient, the component protein molecules of the mixture will exhibit their characteristic charges. The charge of each protein component will be determined by the pI for that protein and the pH range of the gradient corresponding to the position in the electrofocusing column where the protein is initially located. When a constant current is applied to such a system, each protein molecule of the mixture migrates towards the $\mathrm{pH}$ value in the column where its net charge is zero. Each protein of the mixture is therefore resolved at the point in the electrofocusing column where the $\mathrm{pH}$ is equal to the $\mathrm{pI}$.

This process is illustrated in Figure 1l, which schematically depicts the resolution of three proteins marked $\left(\mathrm{pI}_{1}\right),\left(\mathrm{pI} \mathrm{I}_{2}\right)$, and $\left(\mathrm{pI}_{3}\right)$ in an isoelectric focusing column.

\section{The $\mathrm{pH}$ Gradient}

In 1961, Svensson introduced the term "natural pH gradient." Such a pH gradient was termed a natural one, since it is formed by the electric current. Prior to turning on the current, the $\mathrm{pH}$ is constant throughout the solution. A pH gradient in equilibrium cannot be achieved until the current has transported each low-molecular weight ampholyte to its isoelectric point. Since this $\mathrm{pH}$ gradient is in equilibrium, it remains stable for long periods of time, and unlike an artificial pH gradient (obtained by arranging 


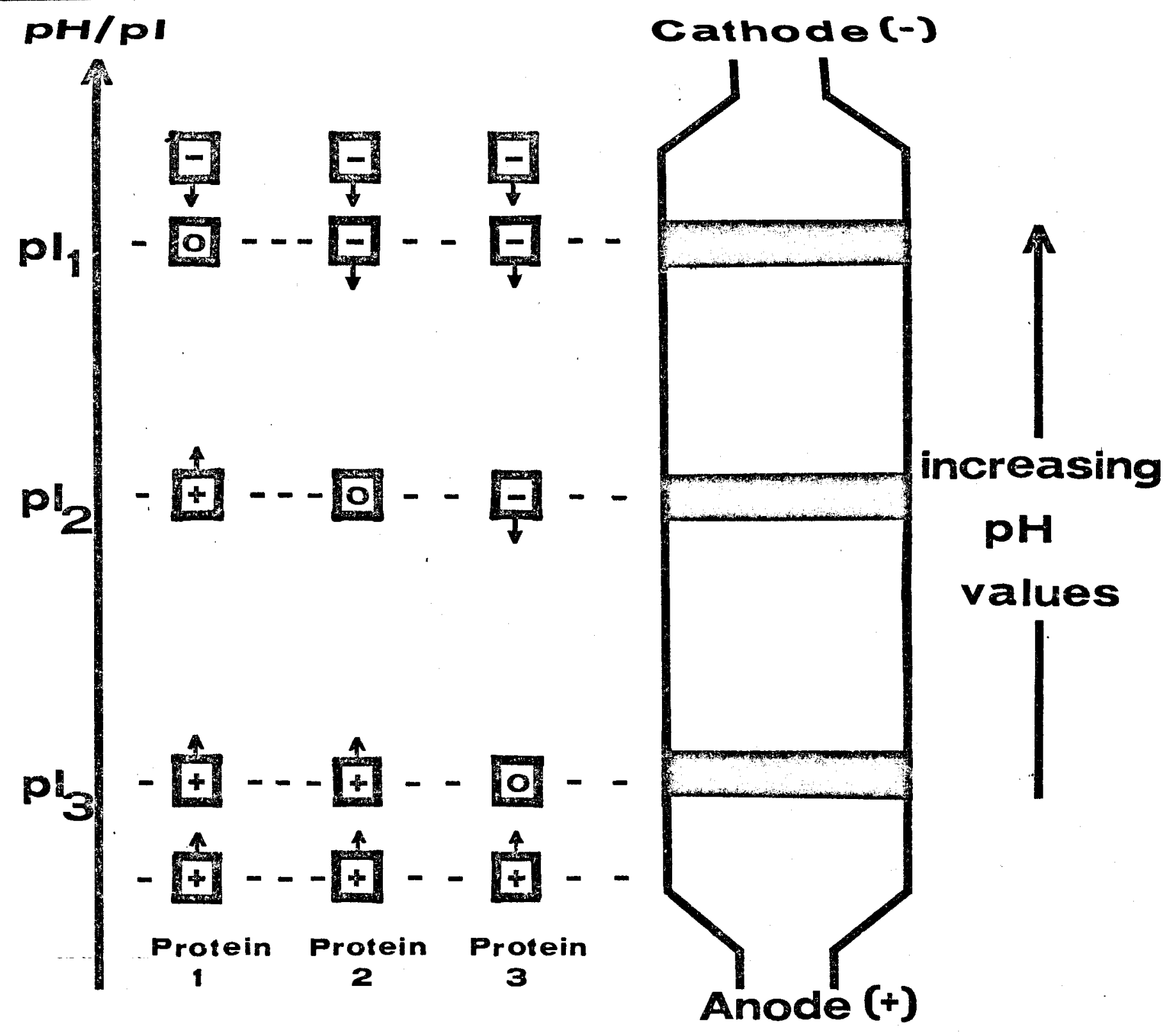

Figure 11. Three proteins marked $\left(p l_{1}\right),\left(p l_{2}\right)$, and $\left(p l_{3}\right)$ are schematically electrofocused in a column. 
buffer solutions of different $\mathrm{pH}$ in series so they can diffuse into each other), it will not migrate. Therefore, the "natural pH gradient" is an ideal medium for isoelectric fractionation of ampholytes such as proteins.

Svensson ${ }^{\circ}$ s theoretical considerations (1961) and fundamental work (1962) defined the required properties of carrier ampholytes which would comprise the natural $\mathrm{pH}$ gradient for isoelectric focusing of proteins. The prerequisites of any carrier ampholytes used for isoelectric fractionation are listed below:

1) A carrier ampholyte should have good buffering capacity at its isoelectric point, so that it is capable of well-defining the $\mathrm{pH}$ at that point and of dominating the high-molecular ampholytes (proteins) which are to move in the zone where it reigns.

2) A good buffering capacity is accompanied by good conductivity at the isoelectric point.

3) The carrier ampholytes should have low molecular weights in order to be separable by dialysis or column chromatography from the high-molecular weight compounds which are to be electrofocused.

4) The carrier ampholytes should also have a chemical composition that is different from the substance to be separated and analyzed, in order that conventional methods of identifi- 
cation and analysis of these substances may be applied without disturbances from the carrier ampholytes.

5) The carrier ampholytes should not react with or denature the substance to be fractionated.

Vesterberg and Svensson (1966) developed a method of synthesizing ampholytes having the characteristics which had been defined by Svensson (1961). These synthetic ampholytes were aliphatic polyamino-polycarboxylic acids exhibiting many values of $\mathrm{pK}$ for the same type of molecule and having great solubility in water. Vesterberg (1969) successfully accomplished the synthesis of carrier ampholytes which were a mixture of many isomers and homologues with different, closely spaced $\mathrm{pK}$ and $\mathrm{pI}$ values, covering the $\mathrm{pH}$ range from 3 to 10. This was accomplished by reacting a mixture of many different polyethylene-polyamines in aqueous solution with acrylic acid. The ampholytes with molecular weights ranging from 300 to 1000 are focused and fractionated in a multicompartment electrolyzer, into groups with closely spaced pI values.

The carrier ampholytes, covering a multitude of $\mathrm{pH}$ ranges, are now commercially available from LKB Instruments, Inc., Rockville, Maryland, under the trade name of Ampholine Carrier Ampholytes. The carrier ampholytes may be characterized by the following general formula: 


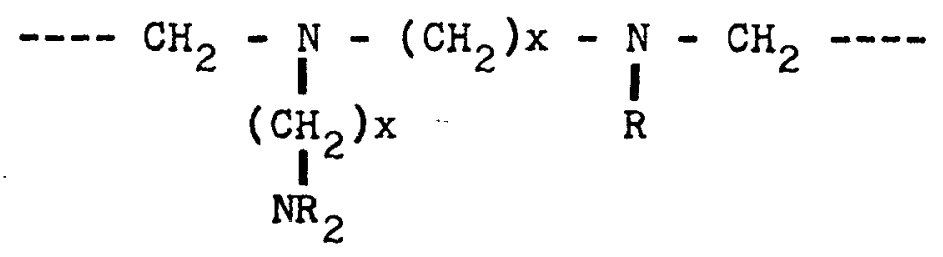

wheres $\mathrm{x}=2$ or 3

and, $\mathrm{R}=\mathrm{H}$ or $-\left(\mathrm{CH}_{2}\right) \mathrm{x}-\mathrm{COOH}$

At the beginning of an isoelectric focusing experiment, the $\mathrm{pH}$ is constant throughout the electrofocusing column and is the average value of all the carrier ampholytes in the solution. Some of the carrier ampholytes are positively charged and others are negatively -charged. When a current is applied to the electrofocusing compartment, these ampholines start migrating towards their respective electrodes. While they are migrating, a pH gradient is successively built up due to their buffering capacity. Each carrier ampholyte will establish a pH range corresponding to its isoelectric point. The carrier ampholytes ater a 24 to 72 hour period will be distributed in a pH gradient which they, themselves, have created in order of their isoelectric points.

The mixed protein sample that is to be separated into its component fractions by the electrofocusing procedure is added to the electrofocusing compartment together with the carrier ampholytes. When the current is applied, the sample proteins will also start migrating toward their respective isoelectric points because of the pH gradient established by the carrier ampholytes. 
The pH range of the Ampholine Carrier Ampholytes should be chosen as narrow as possible when electrofocusing to give the best resolution for each protein. The $\mathrm{pH}$ range should be just large enough to include the $p I$ values of the proteins of interest. When electrofocusing a mixture of proteins for the first time, it is desirable to electrofocus a portion of the protein sample in the broadest $\mathrm{pH}$ range (3-10) before the optimum range can be selected for future electrofocusing experiments.

\section{The Density Gradient}

The carrier ampholytes and the protein sample in the electrofocusing column must be stabilized against convection in some way. A suitable way to accomplish this is to use density gradient of decreasing concentration, from bottom to top, of sucrose or other non-ionic solutes (such as ethylene glycol) dissolved in the electrofocusing solution. The linear sucrose gradient is arranged by filling the electrofocusing column with fractions of decreasing density (carrier ampholytes, sucrose, and sample) or by utilizing an LKB Gradient Mixer (LKB Instruments, Inc., Rockville, Maryland) designed specifically for filling the electrofocusing column.

The gradient mixer gives a linear gradient of two solutions, termed "light" and "dense" (containing sucrose) solutions. The protein mixture is added to one or a few fractions if the density gradient is made manually or in the "light" solution if the LKB Gradient Mixer is utilized. 
Electrode Solutions

The carrier ampholytes located near either electrode must be protected from anodic oxidation and cathodic reduction. This is accomplished by surrounding the anode with a dilute acid solution such as phosphoric acid or sulfuric acid and the cathode with a dilute alkaline solution such as sodium hydroxide or ethanolamine.

During the electrofocusing experiment, the dilute acid and base solutions are drawn to the respective electrodes. At the anode, the dilute acid solution gives a net positive charge to any nearby carrier ampholytes, which are thus repelled from the anode and hence, are protected from electrolytic decomposition. Similarly, the dilute base at the cathode results in the formation of negative ampholytes, thereby causing them to be repelled from the cathode.

The Principle of a Practical Isoelectric Focusing Run

The highly sensitive resolving power of the isoelectric focusing technique can best be illustrated by an electrofocusing experiment in which a mixture of commercially available hemoglobin $A, S$, and C preparations (Travenol Laboratories, Inc., Costa Mesa, California) was resolved and the pI values determined. A similar experimental protocol was utilized to resolve microsomal and standard calcitonin preparations and to determine their pI values.

Ampholine carrier ampholytes having a pH range of 7 to 9 were utilized to resolve a $14 \mathrm{mg}$ mixture of hemoglobin $\mathrm{A}, \mathrm{S}$, and $\mathrm{C}$ into separated fractions whose characteristic pI values at $4^{\circ} \mathrm{C}$ was determined. 
The entire electrofocusing column (LKB 8100 Ampholine Column, IKB Instruments Inc., Rockville, Maryland) is thermostated at $4^{\circ} \mathrm{C}$ by utilizing a cryostat (Tamson Thermostatic Bath (TVM-9) and circulator (PBC-4, Neslab Instruments Inc., Portsmouth, New Hampshire), which circulated coolant around both the inner and outer electrofocusing columns. The coolant consisted of equal volumes of distilled water and ethylene glycol. The central electrode valve was in the open position. The electrode solution for the central electrode, $10 \mathrm{ml}$ of $0.1 \mathrm{~N}$ phosphoric acid, was filled with an LKB 12000-1 varioperpex pump (LKB Instruments Inc., Rockville, Maryland) into the central electrode compartment (containing a teflon rod around which a platinum wire was wrapped). This served as the anode during the isoelectric focusing of the mixture of hemoglobins.

The mixed hemoglobin sample, Ampholine carrier ampholytes of pH range 7 to 9, and sucrose were mixed together in the LKB Gradient Mixer so that the solution (110 ml total) gave a linear density gradient when the electrofocusing compartment was filled with the peristaltic pump over a 1 hour period. The remaining space at the top of the electrofocusing column was filled with $10 \mathrm{ml}$ of $0.1 \mathrm{~N} \mathrm{NaOH}$ so that the upper platinum electrode was completely covered by electrode solution. This served as the cathode in the electrofocusing experiment. A constant voltage ( 400 volts) provided by an electrophoresis power supply (Gelman Instrument Co., Ann Arbor, Michigan) was then applied to the electrofocusing column via the plugs at the central electrode terminal and upper electrode terminal and the isoelectric focusing of the hemoglobin mixture was started. In practice, the best results are obtained by placing the cathode at the top of the 
electrofocusing column for $\mathrm{pH}$ regions below $\mathrm{pH} 6$, and the anode at the top of the column for $\mathrm{pH}$ regions above $\mathrm{pH} 6$. If this practice is not followed, protein precipitation may occur at the top of the column and may disturb focused zones due to settling of the precipitate.

When the current (at a constant voltage of 500 volts) decreased to a constant value of $2 \mathrm{ma}$ all the carrier ampholytes in the electrofocusing column have migrated to their isoelectric points. The hemoglobin $A, S$, and $C$ also have focused at their isoelectric points; however, their contribution to the conductance was extremely low. The electrofocusing run in which hemoglobin $A, S$, and $C$ were resolved, required a 48 hour electrofocusing time. In general, an electrofocusing run normally takes from 24 to 72 hours at a voltage of 300 to 500 volts, depending on the ampholine pH range used. Generally longer electrofocusing times are required for resolution of proteins in narrower pH ranges of ampholines.

At the completion of the electrofocusing procedure, the voltage was turned off and the central electrode valve was closed to prevent the anode electrode solution from mixing with the effluent.

The outlet clamp for the electrofocusing column was then opened and the column was emptied by means of an LKB peristaltic pump. The effluent was monitored with an LKB Absorptiometer at $280 \mathrm{~nm}$. Forty drop fractions (circa $3.6 \mathrm{ml}$ ) were collected in test tubes and the $\mathrm{pH}$ of each fraction was measured at $4^{\circ} \mathrm{C}$.

Figure 12 shows an LKB 8100 Ampholine Electrofocusing Column in which hemoglobin $A, S$, and $C$ have been distinctly resolved. A large number of unidentified minor bands are also shown. This indicates 


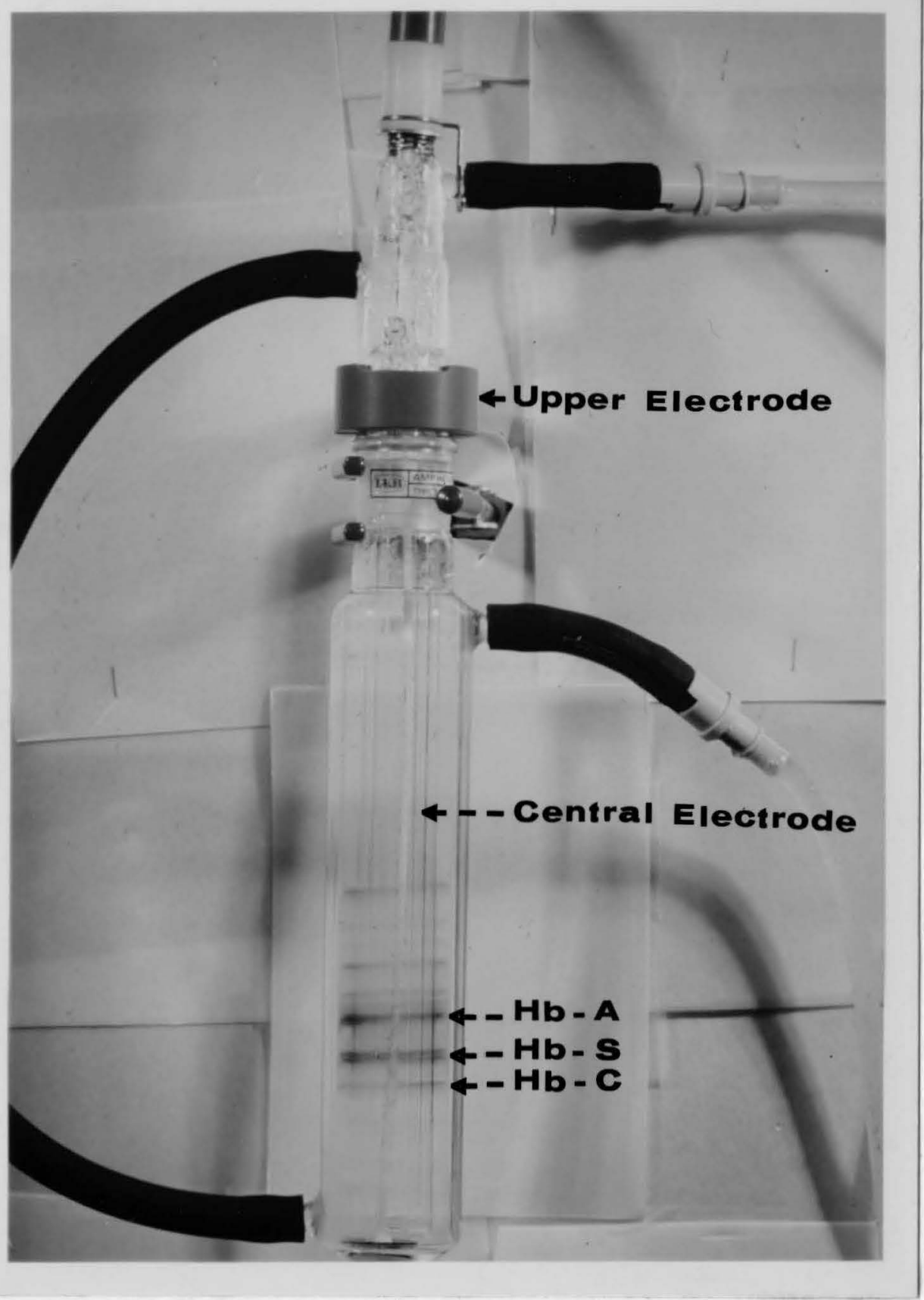

Figure 12. LKB 8100 Electrofocusing Column with completed separation of a mixture of hemoglobins A, S, and $\mathrm{C}$ in a $\mathrm{pH}$ gradient of 7 to 9 . 
the highly sensitive resolving power of the isoelectric focusing technique and clearly demonstrates that commercially "pure" preparations of hemoglobin $A, S$, and $C$ are, in reality, not at all in the pure form.

Figure 13 depicts the absorption curve and superimposed $\mathrm{pH}$ curve obtained by eluting the electrofocused $A, S$, and $C$ hemoglobin bands from the column. The $\mathrm{pH}$ of the collected fraction at the elution peaks corresponds to the pI of the various resolved hemoglobin components. 
PH 8.0

7.9

Hb-A

7.8

7.7 Ancole

Electrode

7.6 Solution

$7.5 \stackrel{1}{7}$

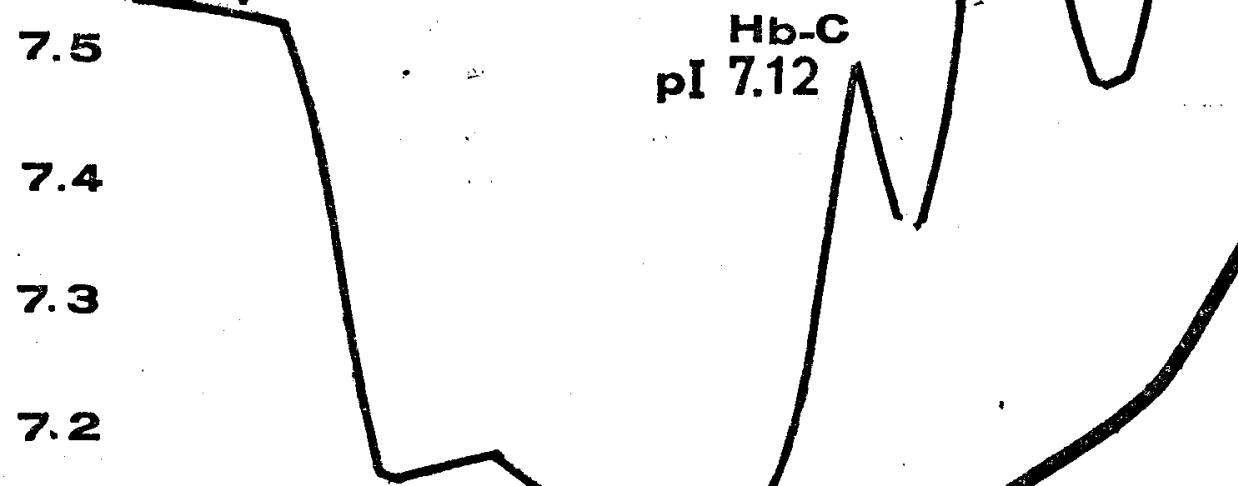

7.1

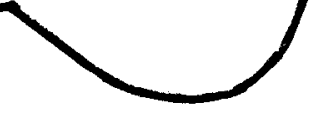

Hb-S

pI 7.32

7.2

7.0

36

54

$72 \mathrm{ml}$

30

27

$23 \quad 22$

20

18

pI 7.63

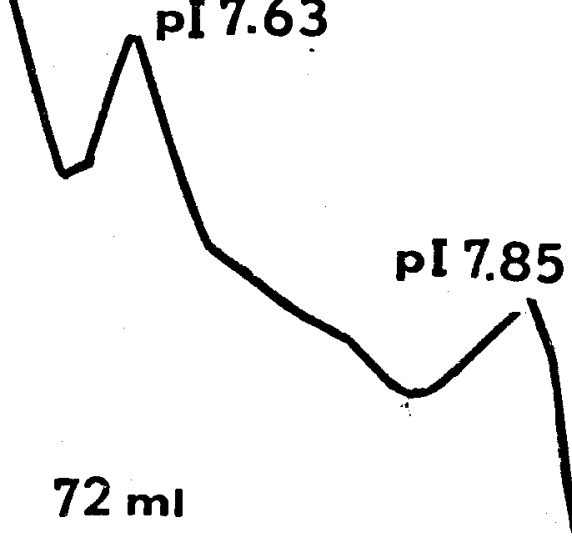

$15 \mathrm{~cm}$

FIGURE 13. Separation of hemoglobin A, S, and C mixture by the electrofocusing apparatus shown in Figure. The curve with peaks shows the absorption in the eluate at $280 \mathrm{~nm}$. The steadily increasing curve is a plot of the pH gradient superimposed. Isoelectric point values shown at the various peaks were read from the $\mathrm{pH}$ gradient curve. 


\section{CHAPTER III}

\section{EXPERIMENTAL RESULTS}

The experimental portion of this dissertation consists of three parts. The first part deals with the subcellular localization of calcitonin and the influence of calcium concentration on the biosynthesis of the hormone in subcellular fractions. Ovine thyroid gland slices were incubated in an in vitro biosynthetic medium consisting of Krebs-Ringer bicarbonate buffer ( $\mathrm{pH} 7.4)$ containing glucose, all the amino acids of ovine calcitonin, and in many instances, glycine-C-14 (UL), which served as a tracer amino acid. The calcium concentration of the incubation medium was the only parameter which was varied. Low calcium concentration incubation medium contained $4.8 \mathrm{mg}$ of calcium/l00 ml, the physiological, $11.5 \mathrm{mg}$ of calcium/100 ml, and the high, $26.0 \mathrm{mg}$ of calcium/ $100 \mathrm{ml}$. After incubation, the slices were homogenized and mitochondrial, nuclear, and microsomal subcellular fractions were obtained by differential centrifugation. Aliquots of these three subcellular preparations obtained from incubation media containing the varying calcium concentrations were injected into rats and assayed for hypocalcemic activity.

The second part of this report concerns establishing the identity of the hypocalcemic principle in the subcellular fraction by comparison with a standard calcitonin preparation and determining the homogeneity of the hypocalcemic entity at each stage 
of hormone purification. This was accomplished by electrophoretic fractionation of the hypocalcemic preparations and of standard calcitonin preparations of known potency, in polyacrylamide gels. The hypocalcemic fraction was inspected for homogeneity in polyacrylamide gels after passage through Sephadex G-100 and G-50 chromatographic columns.

The last part of the experimental work to be described deals with the further characterization of the hypocalcemic subcellular and standard calcitonin preparations by determining a significant physical constant of this hypocalcemic principle, its isoelectric point ( $\mathrm{pI}$ ) by means of isoelectric focusing. Moreover, this technique permitted resolution of the hypocalcemic fraction into discrete protein bands which could be recovered in milligram quantities and assayed for hypocalcemic activity.

THE INFLUENCE OF INCR EASING THE LOSE OF THE SUBCELLULAR FRACTION

ON HYPOCALCENIC ACTIVITY

Protein synthesis occurs on the ribosomes and these subcellular organelles are prominent constituents of the heterogeneous microsomal fraction obtained by differential centrifugation which is composed of smooth and rough endoplasmic reticulum, dense granules, and free ribosomes. Therefore, it seemed quite proper to assay this subcellular fraction first for the hypocalcemic activity associated with this peptide hormone. In order to establish whether or not this microsomal fraction would indeed exhibit hypocalcemic activity, two grams of sheep thyroid slices were 
placed in a series of $50 \mathrm{ml}$ Erlenmeyer flasks which contained 10 ml of a physiological calcium concentration incubation medium, 11.5 mg of calcium per $100 \mathrm{ml}$ of $\mathrm{Krebs}-\mathrm{R}$ inger bicarbonate buffer. The ovine thyroid slices were incubated for four hours at $37^{\circ} \mathrm{C}$ in a constant temperature shaker water bath. The incubation was terminated by placing the flasks in an ice-bath. The slices were immediately homogenized and a microsomal subcellular fraction was obtained by differential centrifugation. The fresh microsomal preparation was immediately lyophilized. For hypocalcemic determinations, various amounts of the microsomal material were dissolved in $250 \mu \mathrm{l}$ of deionized water which served as the vehicle. Microsomal material in concentrations of $250 \mu \mathrm{g}, 1,000 \mu \mathrm{g}$ and 2,000 $\mu \mathrm{g}$ per $250 \mu \mathrm{l}$ of deionized water were assayed for hypocalcemic activity in 130 to $160 \mathrm{~g}$ Holtzman rats which were fasted for $18 \mathrm{hr}$ before their use for biological assay. This was done first of all, to establish whether or not this fraction was hypocalcemic, and secondly, to determine the injection dose which would result in a maximal hypocalcemic response. The various concentrations of microsomal material were injected intravenously into rats and the calcium concentration of the serum samples was determined before and 50 minutes after injection.

The microsomal preparations were analyzed for protein content and the change in serum calcium was expressed as the change in calcium in $\mathrm{mg} \% / 10 \mu \mathrm{g}$ protein. The influence on hypocalcemic activity of increasing microsomal injection doses is presented in Table VII. It is apparent that intravenous injection of as little as $250 \mu \mathrm{g}$ of crude microsomal material evoked a hypocalcemic re- 


\section{TABLE VII}

HYPOCALCEMIC ACTIVITY OF INCREASING DOSES OF MICROSOMAL PREPARATIONS OBTAINED FROM INCUBATION MEDIA CONTAINING PHYSIOLOGICAL CALCIUM CONCENTRATIONS

\begin{tabular}{|c|c|c|c|c|c|c|}
\hline $\begin{array}{c}\text { Dose } \\
\mu \mathrm{g}\end{array}$ & $\begin{array}{l}\text { Animal } \\
\text { No. }\end{array}$ & $\frac{\mathrm{Ca}}{\mathrm{t}_{\mathrm{o}}}$ & $\frac{\mathrm{mg} \%}{t_{50}}$ & $\begin{array}{l}\Delta \mathrm{Ca} \text { mg\%/ } \\
10 \mu \mathrm{g} \text { Protein }\end{array}$ & $\begin{array}{l}\text { Net } \\
\text { Change }\end{array}$ & $\stackrel{\%}{\%}$ \\
\hline \multirow[t]{7}{*}{$250 \mathrm{~kg}$} & 1 & 10.40 & 8.50 & -0.101 & -1.90 & -18.3 \\
\hline & 2 & 10.10 & 9.68 & -0.022 & -0.42 & -4.2 \\
\hline & 3 & 10.90 & 10.40 & -0.027 & -0.50 & -4.6 \\
\hline & 4 & 9.50 & 9.30 & -0.011 & -0.20 & -2.1 \\
\hline & 5 & 11.35 & 10.10 & -0.066 & -1.25 & -11.0 \\
\hline & 6 & 10.20 & 9.80 & -0.021 & -0.40 & -3.9 \\
\hline & \multicolumn{2}{|c|}{ Mean $\pm S . D$} & & \multicolumn{2}{|c|}{$-0.041 \pm 0.031$} & -7.4 \\
\hline \multirow{6}{*}{$1,000 \mathrm{\mu g}$} & 1 & 14.00 & 7.95 & -0.173 & -6.05 & -43.2 \\
\hline & 2 & 14.20 & 11.60 & -0.074 & -2.60 & -18.3 \\
\hline & 3 & 10.00 & 7.60 & -0.069 & -2.40 & -24.0 \\
\hline & 4 & 12.00 & 11.30 & -0.020 & -0.70 & -5.8 \\
\hline & 5 & 8.10 & 7.70 & -0.011 & -0.40 & -4.9 \\
\hline & \multicolumn{2}{|c|}{ Mean \pm S. D. } & & \multicolumn{2}{|c|}{$-0.069 \pm 0.057$} & -19.2 \\
\hline \multirow{8}{*}{$\underline{2,000 ~ \mu g}$} & 1 & 13.60 & 8.35 & -0.151 & -5.25 & -38.6 \\
\hline & 2 & 10.75 & 6.90 & -0.110 & -3.85 & -35.8 \\
\hline & 3 & 11.40 & 8.35 & -0.088 & -3.05 & -26.8 \\
\hline & 4 & 10.50 & 7.70 & -0.080 & -2.80 & -26.7 \\
\hline & 5 & 11.30 & 8.80 & -0.072 & -2.50 & -22.1 \\
\hline & 6 & 11.00 & 9.40 & -0.046 & -1.60 & -14.5 \\
\hline & 7 & 13.30 & 11.60 & -0.048 & -1.70 & -12.8 \\
\hline & \multicolumn{2}{|c|}{ Mean $\pm S$. D. } & & \multicolumn{2}{|c|}{$-0.085 \pm 0.036$} & $-25 \cdot 3$ \\
\hline
\end{tabular}


sponse, lowering the serum calcium by approximately $7 \%$, while injection of $2,000 \mu \mathrm{g}$ of this fraction resulted in a $25 \%$ decrease in serum calcium of rats 50 minutes after injection. A concomitant increase in the specific activity term, mgs \% calcium change/10 $\mu \mathrm{g}$ protein, was also observed; the specific activity increased from -0.041 for the $250 \mu \mathrm{g}$ dose to -0.085 for the $2,000 \mu \mathrm{g}$ preparation. The 2,000 $\mathrm{\mu g}$ microsomal dose elicited a maximal hypocalcemic response. A number of attempts were made to assay a $3,000 \mu \mathrm{g}$ dose for hypocalcemic activity; however, this amount of microsomal material was quite insoluble in deionized water, often resulting in the death of test rats, probably due to obstruction of the circulatory system by the suspension. In the few surviving animals, at this dosage, serum calcium was decreased by approximately the same amount as with the $2,000 \mu \mathrm{g}$ dose. It was therefore decided to utilize a $2,000 \mu \mathrm{g}$ dose of mitochondrial, nuclear, and microsomal preparations for hypocalcemic determinations in all subsequent experiments.

THE INFLUENCE OF PHYSIOLOGICAL AND HIGH CALCIUM CONCENTRATIONS IN THE INCUBATION MEDIUM ON THE HYPOCALCEMIC RESPONSE OF SUBCELLULAR FRACTIONS ISOLATED FROM INCUBATED THYROID SLICES

Although the microsomal fraction exhibited hypocalcemic activity at all dose levels tested, a distinct possibility remained that the mitochondrial and nuclear preparations might also exhibit hypocalcemic activity. Brown and Ulvedal (1960) had localized TSH activity in the mitochondria and ACTH activity was found to be 
dispersed over several subcellular fractions; however, no hormonal activity was found to be present in the nuclear fraction. Yet, one can not arbitrarily dismiss these fractions from any hypocalcemic determinations. Consequently, mitochondrial and nuclear fractions, were obtained from incubation media containing either physiological or high levels of calcium, $11.5 \mathrm{mg} / 100 \mathrm{ml}$ and $26.0 \mathrm{mg} / 100 \mathrm{ml}$, respectively; and were assayed for hypocalcemic activity in rats at a dose of 2,000 $\mathrm{gg}$. In these initial studies, no tracer amino acid (glycine-C-14) was utilized in the incubation media.

A calcium concentration of $11.5 \mathrm{mg} / 100 \mathrm{ml}$ of Krebs-Ringer bicarbonate buffer incubation medium was selected as a physiological calcium concentration, since the normal serum calcium level in most mammals ranges from 9.0 to $12.5 \%$ depending on the test animal. In rats, the normal serum calcium level ranges from 9 to $11.5 \%$, therefore, the $11.5 \%$ calcium concentration was at the upper end of the normal range. The reason for selecting $26.0 \mathrm{mg} / 100 \mathrm{ml}$ of Krebs-Ringer bicarbonate buffer as a high calcium concentration incubation medium was somewhat arbitrary. It is known that in mammals serum calcium levels seldom exceed 22 to $23 \mathrm{mg} \%$ even in severe cases of hyperparathyroidism or experimentally induced hypercalcemia; consequently, the high calcium concentration used here was slightly above the highest reported values for serum calcium. 
The influence of a physiological calcium concentration of the incubation medium on the hypocalcemic response in rats injected with aliquots of mitochondrial and nuclear fractions isolated from the incubated thyroid slices is shown in Table VIII. The hypocalcemic response of the microsomal preparation has already been noted in Table VII. Surprisingly, the nuclear preparation decreased the serum calcium of rats by approximately $27 \%$. This was $2 \%$ more than the $25 \%$ decrease in serum calcium of rats injected with microsomal material. The mitochondrial preparation, however, elicited only a slight hypocalcemic response. Although the hypocalcemic response of the nuclear and microsomal preparations were quite similar, injection of the nuclear preparation resulted in only a $-0.038 \mathrm{mg} \%$ change in serum calcium per $10 \mu \mathrm{g}$ protein whereas the microsomal preparation changed serum calcium by $-0.085 \mathrm{mg} \%$ per $10 \mu \mathrm{g}$ protein. This indicated that the microsomal preparation exhibited over twice the hypocalcemic response per $10 \mu \mathrm{g}$ of protein, as did the nuclear fraction. It appeared likely that the hypocalcemic response of the nuclear fraction could be attributed to significant microsomal contamination of this fraction. This possibility was borne out in further testing.

The influence of a high calcium concentration on the hypocalcemic response of the microsomal, mitochondrial, and nuclear subcellular fractions is shown in Table IX. In this experiment, only the microsomal preparation exhibited a hypocalcemic response, 
TABLE VIII

HYPOCALCEMIC ACTIVITY OF MITOCHONDRIAL AND NUCLEAR PREPARATIONS AT A CONCENTRATION OF 2,000 $\mu \mathrm{g}$ OBTAINED FROM INCUBATION MEDIA CONTAINING PHYSIOLOGICAL CALCIUM CONCENTRATIONS

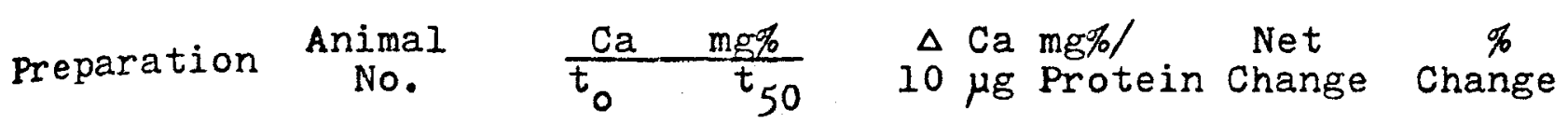

\begin{tabular}{rlrrrrr} 
Mitochondrial & 1 & 8.10 & 8.30 & +0.002 & +0.20 & +2.5 \\
2 & 7.80 & 8.50 & +0.006 & +0.70 & +9.0 \\
3 & 8.10 & 7.00 & -0.010 & -1.10 & -13.6 \\
4 & 7.40 & 7.60 & +0.002 & +0.20 & +2.7 \\
5 & 8.10 & 7.50 & -0.005 & -0.60 & -7.4 \\
6 & 8.50 & 7.80 & -0.006 & -0.70 & -8.2 \\
7 & 8.50 & 7.60 & -0.008 & -0.90 & -10.6 \\
\hline Nuclear & Mean \pm S. D. & & $-0.003 \pm 0.000$ & -3.7 \\
\hline 1 & 12.40 & 7.90 & -0.066 & -4.50 & -36.3 \\
2 & 9.50 & 6.50 & -0.044 & -3.00 & -31.6 \\
3 & 10.40 & 6.00 & -0.064 & -4.40 & -42.3 \\
4 & 9.60 & 9.65 & +0.001 & +0.05 & 0.0 \\
5 & 9.80 & 7.55 & -0.018 & -2.25 & -23.0 \\
\hline Mean \pm S. D. & & $-0.038 \pm 0.028$ & -26.6
\end{tabular}


TABLE IX

HYPOCALCEMIC ACTIVITY OF MICROSOMAL, MITOCHONDRIAL, AND NUCLEAR PREPARATIONS AT A CONCENTRATION OF 2,000 $\mu \mathrm{g}$ OBTAINED FROM INCUBATION MEDIA CONTAINING HIGH CALCIUM CONCENTRATIONS

\begin{tabular}{|c|c|c|c|c|c|c|c|}
\hline Preparation & $\begin{array}{l}\text { Animal } \\
\text { No. }\end{array}$ & $\frac{\mathrm{Ca}}{\mathrm{t}_{0}}$ & $\frac{\mathrm{mg} \%}{t_{50}}$ & $\begin{array}{r}\Delta \\
10\end{array}$ & $\begin{array}{l}\text { Ca mg\%/ } \\
\mu g \text { Protein }\end{array}$ & $\begin{array}{l}\text { Net } \\
\text { Change }\end{array}$ & $\begin{array}{c}\% \\
\text { Change }\end{array}$ \\
\hline \multirow[t]{8}{*}{ Microsomal } & 1 & 11.84 & 9.41 & & -0.054 & -2.43 & -20.5 \\
\hline & 2 & 10.74 & 8.54 & & -0.049 & -2.20 & -20.5 \\
\hline & 3 & 10.62 & 8.74 & & -0.042 & -1.88 & -17.7 \\
\hline & 4 & 10.39 & 7.97 & & -0.054 & -2.42 & $-23 \cdot 3$ \\
\hline & 5 & 10.92 & 7.97 & & -0.066 & -2.95 & -27.0 \\
\hline & 6 & 10.06 & 8.94 & & -0.025 & -1.12 & -11.1 \\
\hline & 7 & 10.06 & 8.45 & & -0.036 & -1.61 & -16.0 \\
\hline & \multicolumn{2}{|c|}{ Mean \pm S. D. } & & & \multicolumn{2}{|c|}{$-0.047 \pm 0.010$} & -19.4 \\
\hline \multirow[t]{8}{*}{ Mitochondrial } & 1 & 10.39 & 8.78 & & -0.034 & -1.61 & -15.5 \\
\hline & 2 & 9.20 & 10.79 & & +0.034 & +1.59 & +17.3 \\
\hline & 3 & 8.11 & 10.00 & & +0.040 & +1.89 & +23.3 \\
\hline & 4 & 8.45 & 10.05 & & +0.034 & +1.60 & +18.9 \\
\hline & 5 & 8.94 & 10.11 & & +0.025 & +1.17 & +13.1 \\
\hline & 6 & 8.94 & 9.19 & & +0.005 & +0.25 & +2.8 \\
\hline & 7 & 9.58 & 9.46 & & -0.003 & -0.12 & -1.3 \\
\hline & \multicolumn{2}{|c|}{ Mean $\pm S . D$} & & & $+0.014 \pm 0$ & 0.026 & +8.4 \\
\hline \multirow[t]{8}{*}{ Nuclear } & 1 & 11.24 & 8.06 & & -0.150 & -3.18 & -28.3 \\
\hline & 2 & 8.45 & 9.84 & & +0.066 & +1.39 & +16.4 \\
\hline & 3 & 8.74 & 9.09 & & +0.017 & +0.35 & +4.0 \\
\hline & 4 & 9.10 & 8.99 & & -0.005 & -0.11 & -1.2 \\
\hline & 5 & 8.59 & 10.11 & & +0.072 & +1.52 & +17.7 \\
\hline & 6 & 8.20 & 8.94 & & +0.035 & +0.74 & +9.0 \\
\hline & 7 & 8.79 & 10.28 & & +0.070 & +1.49 & +17.0 \\
\hline & \multicolumn{2}{|c|}{ Mean $\pm S . D}$. & & & \multicolumn{2}{|c|}{$+0.015 \pm 0.078$} & +4.9 \\
\hline
\end{tabular}


10wering serum calcium of rats by approximately $19 \%$. The mitochondrial and nuclear preparations elicited a slight hypercalcemic response in rats, raising the serum calcium levels by 8.4 and $4.9 \%$ respectively. No hypocalcemic response was noted in rats injected with the nuclear preparation obtained from incubation media containing a high calcium concentration. This was in contrast to the hypocalcemic response of the nuclear fraction obtained from an incubation medium containing a physiological calcium concentration as was noted in Table VIII, probably indicative of significant microsomal contamination in this fraction. Both the mitochondrial and nuclear preparations raised the serum calcium by approximately $+0.015 \mathrm{mg} \%$ per $10 \mu \mathrm{g}$ protein while the microsomal preparation decreased serum calcium by $-0.047 \mathrm{mg} \%$ per $10 \mu \mathrm{g}$ protein, clearly indicating that the microsomal preparation contained the subcellular locus for the hypocalcemic hormone.

Tashjian and Warnock (1967) demonstrated that incubation of crude porcine calcitonin in $0.03 \mathrm{~N} \mathrm{HC1}$ at $110 \mathrm{C}$ for 18 hours was accompanied by gradual loss of hypocalcemic activity, whereas heating this preparation at $110^{\circ} \mathrm{C}$ for 2 hours in $0.1 \mathrm{~N} \mathrm{HCl}$ resulted in a more rapid inactivation of the molecule. However, heating the calcitonin preparation for 15 minutes at $110^{\circ} \mathrm{C}$ did little to alter the hypercalcemic response, thereby indicating the extreme thermostability of their crude CT preparation. Therefore, an attempt was made to test the microsomal preparation which had decreased 
the serum calcium level of rats by $25 \%$ (Table VII) for its thermostability. Heat treatment of this preparation at $100^{\circ} \mathrm{C}$ for 2 hours in 0.01 N $\mathrm{HCl}$ did not lead to inactivation. The data for the attempted heat-inactivation of this microsomal preparation obtained from an incubation medium having a physiological calcium concentration is presented in Table $\mathrm{X}$. When this heat-treated preparation was assayed for hypocalcemic activity, a marked hypocalcemic response was noted. Serum calcium levels of rats receiving this preparation decreased approximately $29 \%$, indicating no loss in hypocalcemic activity and the extreme thermostability of the microsomal preparation.

Tashjian and Warnock (1967) had also shown that the timecourse of inactivation of their crude porcine $\mathrm{CT}$ preparation by numerous proteolytic enzymes was extremely rapid. In order to demonstrate a similar effect with the hypocalcemic microsomal preparation, it was hydrolyzed for one hour at $37^{\circ} \mathrm{C}$ with pepsin at $\mathrm{pH} 2.7$. The substrate:enzyme weight ratio was $10: 1$. As can be seen from Table XI, pepsin was extremely effective in destroying the hypocalcemic activity of the microsomal preparation. Now, instead of lowering serum calcium by approximately 25\% (Table VII), injection of this pepsin-treated microsomal preparation resulted in approximately a $4 \%$ increase in serum calcium, or a change in calcium from $-0.085 \mathrm{mg} \%$ per $10 \mu \mathrm{g}$ protein to $-0.001 \mathrm{mg} \%$ per $10 \mu \mathrm{g}$ protein. This experiment indicated the protein nature of this 
TABIE X

HYPOCAICEMIC ACTIVITY OF HEAT-TREATED MICROSONAI PREPARATION AT A CONCENTRATION OF $2,000 \mu \mathrm{g}$ OBTAINED FROM INCUBATION MEDIA CONTAINING PHYSIOLOGICAL CALCIUM CONCENTRATIONS

\begin{tabular}{|c|c|c|c|c|c|c|c|}
\hline Preparation & $\begin{array}{c}\text { Animal } \\
\text { No. }\end{array}$ & $\frac{\mathrm{Ca}}{t_{0}}$ & $\frac{m g \%}{t_{50}}$ & $\begin{array}{r}\Delta \\
10\end{array}$ & $\begin{array}{l}\mathrm{Ca} \text { mg\%/ } \\
\mu g \text { Protein }\end{array}$ & $\begin{array}{l}\text { Net } \\
\text { Change }\end{array}$ & $\begin{array}{c}\% \\
\text { Change }\end{array}$ \\
\hline \multirow[t]{6}{*}{ Microsomal* } & 1 & 10.40 & 5.40 & & -0.148 & -5.00 & -48.1 \\
\hline & 2 & 10.10 & 9.25 & & -0.025 & -0.85 & -8.4 \\
\hline & 3 & 7.90 & 6.00 & & -0.056 & -1.90 & $-24 \cdot 1$ \\
\hline & 4 & 11.20 & 6.55 & & -0.138 & -4.65 & $-41 \cdot 5$ \\
\hline & 5 & $11 \cdot 50$ & 10.45 & & -0.031 & -1.05 & -9.1 \\
\hline & 6 & 13.40 & $7 \cdot 50$ & & -0.175 & $-5 \cdot 90$ & $-44 \cdot 0$ \\
\hline & Mea & S. D. & & & $-0.096 \pm 0$. & 066 & -29.2 \\
\hline
\end{tabular}

* Microsomal preparation heated for 2 hours at $100^{\circ} \mathrm{C}$ in $0.01 \mathrm{~N} \mathrm{HCI}$. 


\section{TABLE XI}

HYPOCALCEMIC ACTIVITY OF PEPSIN-TREATED MICROSOMAL PREPARATION AT

A CONCENTRATION OF $2,000 \mu \mathrm{g}$ OBTAINED FROM INCUBATION MEDIA

CONTAINING PHYSIOLOGICAL CALCIUM CONCENTRATIONS

\begin{tabular}{|c|c|c|c|c|c|c|c|}
\hline Preparation & $\begin{array}{c}\text { Animal } \\
\text { No. }\end{array}$ & $\frac{\mathrm{Ca}}{\mathrm{t}_{\mathrm{o}}}$ & $\frac{m g \%}{t_{50}}$ & $\begin{array}{r}\Delta \\
10\end{array}$ & $\begin{array}{l}\mathrm{Ca} \text { mg\%/ } \\
\mu \mathrm{g} \text { Protein }\end{array}$ & $\begin{array}{l}\text { Net } \\
\text { Change }\end{array}$ & Change \\
\hline \multirow[t]{6}{*}{ Microsomal* } & 1 & 9.30 & 10.00 & & +0.0022 & +0.70 & $+7 \cdot 5$ \\
\hline & 2 & 9.10 & 9.30 & & +0.0006 & +0.20 & +2.2 \\
\hline & 3 & 8.60 & 9.05 & & +0.0014 & +0.45 & +5.2 \\
\hline & 4 & 8.40 & 8.30 & & -0.0003 & -0.10 & $-1 \cdot 2$ \\
\hline & 5 & 8.00 & 8.75 & & +0.0024 & +0.75 & +9.4 \\
\hline & 6 & 8.10 & 8.30 & & -0.0006 & +0.20 & +2.5 \\
\hline & \multicolumn{3}{|c|}{ Mean \pm S. D. } & \multicolumn{3}{|c|}{$-0.001 \pm 0.0000$} & +4.3 \\
\hline
\end{tabular}

* Microsomal preparation hydrolyzed with pepsin for 1 hour at $37^{\circ} \mathrm{C}$ and $\mathrm{pH} 2.7$ at a substratesenzyme weight ratio of 1081 . 
preparation and the sensitivity of the microsomal fraction to inactivation by a proteolytic enzyme.

A statistical analysis employing the unpaired Student's " $t$ " Test was utilized to compare the hypocalcemic response of mitochondrial and nuclear preparations to that of the microsomal preparation. The statistical comparison for the subcellular preparations obtained from incubation media containing physiological and high calcium concentrations is presented in Table XII. A comparison of the change in serum calcium $\mathrm{mg} \%$ per $10 \mu \mathrm{g}$ protein of heattreated microsomal preparation to that of untreated microsomal material from an incubation medium containing a physiological calcium concentration indicated that there was no significant loss in hypocalcemic activity. However, hydrolyzing this same microsomal preparation with pepsin for one hour resulted in a highly significant decline of hypocalcemic activity of this preparation when compared to untreated microsomes. Moreover, a significant difference in hypocalcemic activity was noted when the mitochondrial and the nuclear preparation were compared to the microsomal preparation obtained from an incubation medium containing a physiological calcium ion concentration. Specific activity values (change in serum calcium in $\mathrm{mg} \% / 10 \mu \mathrm{g}$ protein) of the three subcellular fractions obtained from incubation media containing high calcium concentrations were also subjected to statistical analysis (Table XII). Once again, a significant difference in hypocalcemic activity was noted 


\section{TABLE XII}

HYPOCALCEMIC ACTIVITY OF MICROSOMAL, MITOCHONDRIAL, AND NUCLEAR PREPARATIONS OBTAINED FROM INCUBATION MEDIA CONTAINING HIGH AND PHYSIOLOGICAL CALCIUM CONCENTRATIONS

Preparation

$\triangle \mathrm{Ca} \mathrm{mg} \% /$

$10 \mu g$ Protein
$\%$ Change

Physiological

Microsomal

Microsomal**

Microsomal***

Mitochondrial

Nuclear

$$
\begin{aligned}
& -0.085 \pm 0.036-25.3 \\
& -0.096 \pm 0.059-29.2 \\
& +0.001 \pm 0.000+4.3 \quad 0.001 \\
& -0.003 \pm 0.000 \quad-3.7 \\
& 0.001 \\
& -0.038 \pm 0.028 \\
& -26.6 \\
& 0.05
\end{aligned}
$$

$\underline{\text { High }}$
Microsomal
$-0.046 \pm 0.010$
$-19.4$
Mitochondrial
$+0.015 \pm 0.026$
$+8.4$
0.001
Nuclear
$+0.015 \pm 0.078$
$+4.9$
0.05

* Probability of the significance of the difference of the means as determined by the use of an unpaired Student's " $t$ " Test.

** Heat-treated microsomal preparation $\left(2 \mathrm{hr}, 100^{\circ} \mathrm{C}\right.$ and $0.01 \mathrm{~N}$ HCl).

*** Microsomal preparation hydrolyzed by pepsin for 1 hour at $37^{\circ} \mathrm{C}$ and $\mathrm{pH} 2.7$. 
when the mitochondrial and the nuclear preparations were compared to the hypocalcemic response of the microsomal preparation obtained from a medium containing a high calcium concentration.

THE INFLUENCE OF VARYING CALCIUM CONCENTRATIONS IN THE INCUBATION MEDIUM ON THE HYPOCALCEMIC RESPONSE OF SUBCELLULAR PREPARATIONS ISOLATED FROM INCUBATED THYROID SLICES AND ON THE EXTENT OF INCORPORATION OF GLYCINE-C-14 INTO THESE FRACTIONS

The first series of experiments described above dealt with determining the subcellular locus of $\mathrm{CT}$, defining the injection dose which would elicit a maximal hypocalcemic response, evaluating the thermostability of the molecule, and establishing its protein nature. These mitochondrial, microsomal and nuclear preparations were assayed for hypocalcemic activity without further purification.

In this series of experiments, the incorporation of glycine-

C-14 (UL) into subcellular fractions obtained from incubation media containing physiological, low, and high calcium concentrations was an additional parameter that was monitored. In addition, the subcellular preparations were subjected to a purification step. This consisted in passing the fractions through a column of Sephadex G-100 which eliminated a large portion of the membranous macromolecular material associated with each subcellular fraction. 
Sorgente (1969) had demonstrated that a very high calcium concentration $(45 \mathrm{mg} / 100 \mathrm{ml})$ in the incubation media was accompanied by an increase in both synthesis and release of CT from ovine thyroid slices. It was therefore a possibility that a tracer amino acid, such as glycine-C-14 (one of the prominent amino acids of ovine $\mathrm{CT}$ ), could be incorporated preferentially into the subcellular fraction exhibiting hypocalcemic activity. It was also possible that the concentration of calcium in the biosynthetic media could influence the extent of incorporation of label into the hypocalcemic fraction. Consequently, in vitro biosynthetic media were prepared as described previously; however, in this instance, uniformly labeled glycine containing $2.5 \mu \mathrm{Ci}$ of carbon-14 was added to each biosynthetic medium.

Microsomal, mitochondrial, and nuclear preparations obtained from incubation media containing physiological, low, and high calcium concentrations were assayed for hypocalcemic activity, protein content and radioactivity. The hypocalcemic response of purified microsomal, mitochondrial, and nuclear preparations obtained from an incubation medium containing a physiological calcium concentration is shown in Table XIII. The microsomal preparation was the only fraction which exhibited hypocalcemic activity, lowering serum calcium levels of rats by $11.5 \%$. The mitochondrial and nuclear preparations were slightly hypercalcemic, raising serum calcium levels by $1.0 \%$ and $5.3 \%$ respectively. The microsomal preparation 
TABLE XIII

HYPOCALCEMIC ACTIVITY OF MICROSOMAL, MITOCHONDRIAL, AND NUCLEAR PREPARATIONS OBTAINED FROM INCUBATION MEDIA CONTAINING PHYSIOLOGICAL CALCIUM CONCENTRATIONS AND $2.5 \mu \mathrm{Ci}$ OF GLYCINE-C-14 (UL)

\begin{tabular}{|c|c|c|c|c|c|c|c|}
\hline Preparation & $\begin{array}{c}\text { Animal } \\
\text { No. }\end{array}$ & $\frac{\mathrm{Ca}}{\mathrm{t}_{0}}$ & $\frac{m g \%}{t_{50}}$ & $\begin{array}{r}\Delta \\
10\end{array}$ & $\begin{array}{l}\text { Ca mg\%/ } \\
\mu g \text { Protein }\end{array}$ & $\begin{array}{l}\text { Net } \\
\text { Change }\end{array}$ & $\begin{array}{c}\% \\
\text { Change }\end{array}$ \\
\hline \multirow[t]{8}{*}{ Microsomal } & 1 & 11.10 & 10.00 & & -0.023 & -1.10 & -9.9 \\
\hline & 2 & 10.40 & 8.00 & & -0.050 & -2.40 & $-23 \cdot 1$ \\
\hline & 3 & $9 \cdot 90$ & 8.30 & & -0.034 & -1.60 & -16.2 \\
\hline & 4 & 8.80 & $7 \cdot 25$ & & -0.033 & -1.55 & -17.6 \\
\hline & 5 & 10.15 & 10.15 & & 0.000 & 0.00 & 0.0 \\
\hline & 6 & $10 \cdot 30$ & $9 \cdot 90$ & & -0.008 & -0.40 & -3.9 \\
\hline & 7 & 10.05 & 9.05 & & -0.021 & -1.00 & -10.0 \\
\hline & \multicolumn{2}{|c|}{ Mean \pm S. D. } & & & $-0.024 \pm 0$. & 017 & -11.5 \\
\hline \multirow[t]{8}{*}{ Mitochondrial } & 1 & 10.40 & 10.00 & & -0.037 & -0.40 & -3.8 \\
\hline & 2 & 8.60 & 9.00 & & +0.037 & +0.40 & +4.7 \\
\hline & 3 & 8.60 & 9.00 & & +0.037 & +0.40 & +4.7 \\
\hline & 4 & 9.00 & 9.20 & & +0.018 & +0.20 & $+2 \cdot 2$ \\
\hline & 5 & 9.60 & 9.10 & & -0.046 & -0.50 & -5.2 \\
\hline & 6 & 9.60 & 9.80 & & +0.018 & +0.20 & +2.1 \\
\hline & 7 & 9.90 & 10.15 & & +0.023 & +0.25 & $+2 \cdot 5$ \\
\hline & Mean & $\pm S . D_{0}$ & & & $+0.007 \pm 0$ & .005 & +1.0 \\
\hline \multirow[t]{8}{*}{ Nuclear } & 1 & 9.95 & 9.60 & & -0.031 & -0.35 & -3.5 \\
\hline & 2 & 9.45 & 10.15 & & +0.063 & +0.70 & +7.4 \\
\hline & 3 & $9 \cdot 30$ & 10.50 & & $+0.10 ?$ & +1.20 & +12.9 \\
\hline & 4 & 8.50 & 9.40 & & +0.080 & +0.90 & +10.6 \\
\hline & 5 & 9.95 & 10.10 & & +0.013 & +0.15 & +1.5 \\
\hline & 6 & 9.80 & 10.20 & & +0.036 & +0.40 & +4.1 \\
\hline & 7 & 9.90 & 10.60 & & +0.063 & +0.70 & +7.1 \\
\hline & $\begin{array}{l}8 \\
9\end{array}$ & $\begin{array}{l}10.30 \\
10.45\end{array}$ & $\begin{array}{l}11.10 \\
10.50\end{array}$ & & $\begin{array}{l}+0.071 \\
+0.004\end{array}$ & $\begin{array}{l}+0.80 \\
+0.05\end{array}$ & $\begin{array}{l}+7.8 \\
+0.5\end{array}$ \\
\hline \multicolumn{5}{|c|}{ Mean \pm S. D. } & $+0.045 \pm 0$ & .042 & +5.3 \\
\hline
\end{tabular}


decreased serum calcium by $-0.024 \mathrm{mg} \%$ per $10 \mu \mathrm{g}$ of protein while both the mitochondrial and nuclear preparations slight$1 y$ increased serum calcium levels by +0.007 and +0.045 per $10 \mu \mathrm{g}$ protein, respectively. The microsomal preparation which had been purified by passage through a Sephadex G-100 column actually exhibited less hypocalcemic activity per 10 $\mu g$ of protein $(-0.024)$ than the unpurified microsomal preparation $(-0.085$, Table VII). It is likely that a large percentage of the stored $C T$ in this fraction is intimately associated with membranous material that was unable to pass through the chromatographic column. This could account for the smalier hypocalcemic response (11.5\%), of purified microsomal preparation as indicated in Table XIII as compared to a $25.3 \%$ decrease in serum calcium of unpurified microsomes indicated in Table VII.

The elution pattern, protein content, and radioactivity of a microsomal preparation obtained from an incubation medium containing a physiological calcium concentration and passed through a column of Sephadex G-100 is presented in Figure 14. The six to seven fractions representing the elution peak were pooled for the assay of hypocalcemic activity noted above. In this instance, the protein and radioactive peaks corresponded closely to the elution peak. The radioactive peak corresponded to approximately $2300 \mathrm{DPM} / \mathrm{mg}$ protein and the microsomal protein peak contained approximately $250 \mu \mathrm{g}$ protein / $\mathrm{ml}$ of eluent. Figure 15 shows the elution 


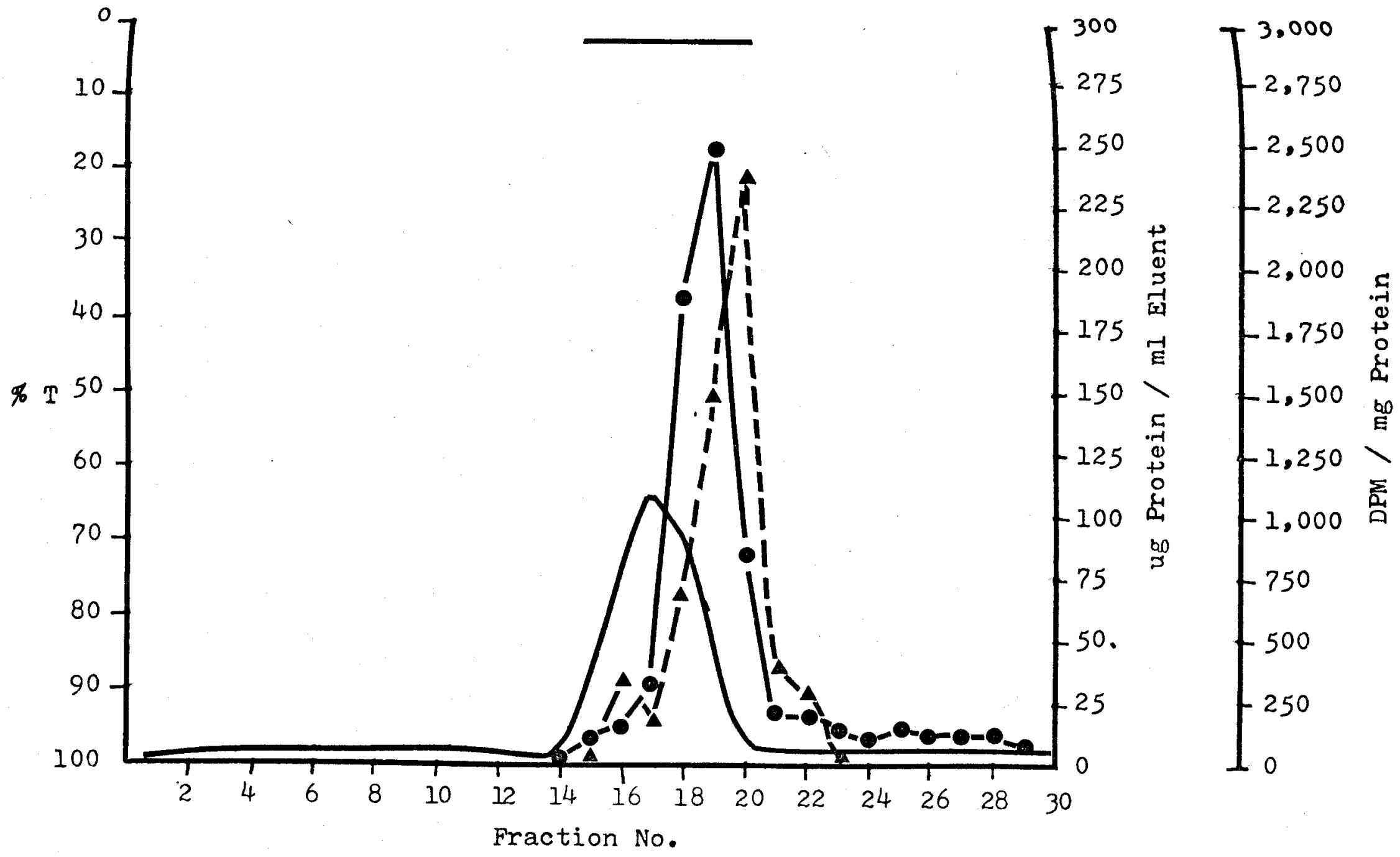

Figure 14. Protein content (- - ) , radioactivity ( $\left.-\mathbf{C}_{-}-\Delta\right)$, and elution pattern ( $(1)$ ) of lyophilized microsomal preparation from a physiological calcium concentration incubation medium passed through a column of sephadex G-100. Flow rate: $35 \mathrm{ml} / \mathrm{hr}$. $4 \mathrm{ml}$ fractions collected. Fractions assayed for hypocalcemic activity indicated by a horizontal bar (- Two times the expansion of the $\%$. T scale. 


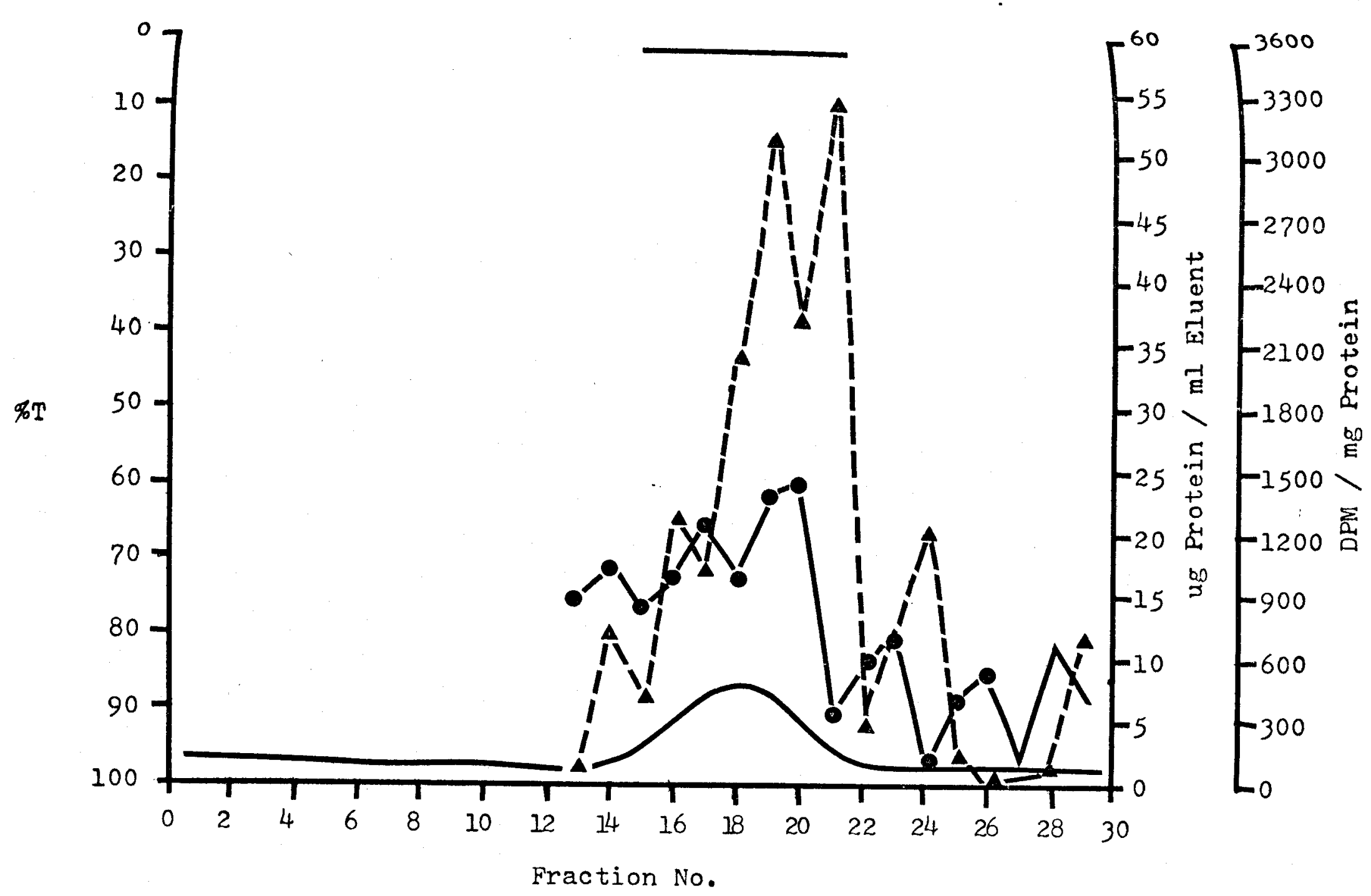

Figure 15. Protein content ( - - $)$, radioactivity $(\Delta--\Delta)$, and elution pattern ( $)$ of lyophilized mitochondrial preparation from a physiological calcium concentration incubation medium passed through a column of Sephadex G-100. Flow rate - $35 \mathrm{~m} / \mathrm{hr} ; 4 \mathrm{ml}$ fractions collected. Fractions assayed for hypocalcemic activity indicated by a horizontal bar ( _ $~$
of the $\% \mathrm{~T}$ scale. 
pattern, protein content, and incorporation of glycine-C-14 into a mitochondrial preparation obtained from the same incubation medium as the previously cited microsomal preparation. The elution, protein, and radioactive peaks correspond rather closely. However, the protein peak of the mitochondrial preparation, $25 \mu \mathrm{g}$ per $\mathrm{m} 1$ eluent, was about 10 times less than the $250 \mu \mathrm{g}$ protein/ml eluent of the microsomal preparation (Figure 14). This was not an unexpected result since it has been well established that protein synthesis occurs on the ribosomes, which are constituents of the microsomal fraction. However, the radioactive peak of this mitochondrial preparation representing $3300 \mathrm{DPM} / \mathrm{mg}$ protein was actually somewhat higher than the radioactive peak of the microsomal preparation representing $2300 \mathrm{DPM} / \mathrm{mg}$ protein as indicated in Figure 14 . This seemed to rule out the possibility that glycine-C-14 was preferentially incorporated into the hypocalcemic microsomal fraction. Similar data were observed with the nuclear preparation which was obtained from an incubation medium containing a physiological calcium concentration as shown in Figure 16. The elution, protein, and radioactivity peaks overlapped quite closely. The protein peak, corresponding to approximately $20 \mu \mathrm{g}$ of protein/ml eluent, was much less than the protein peak of the microsomal preparation. However, the peaks of radioactivity of the nuclear and microsomal preparations were of similar magnitude, being approximately 2700 DPM per mg of protein for the nuclear preparation as compared to 


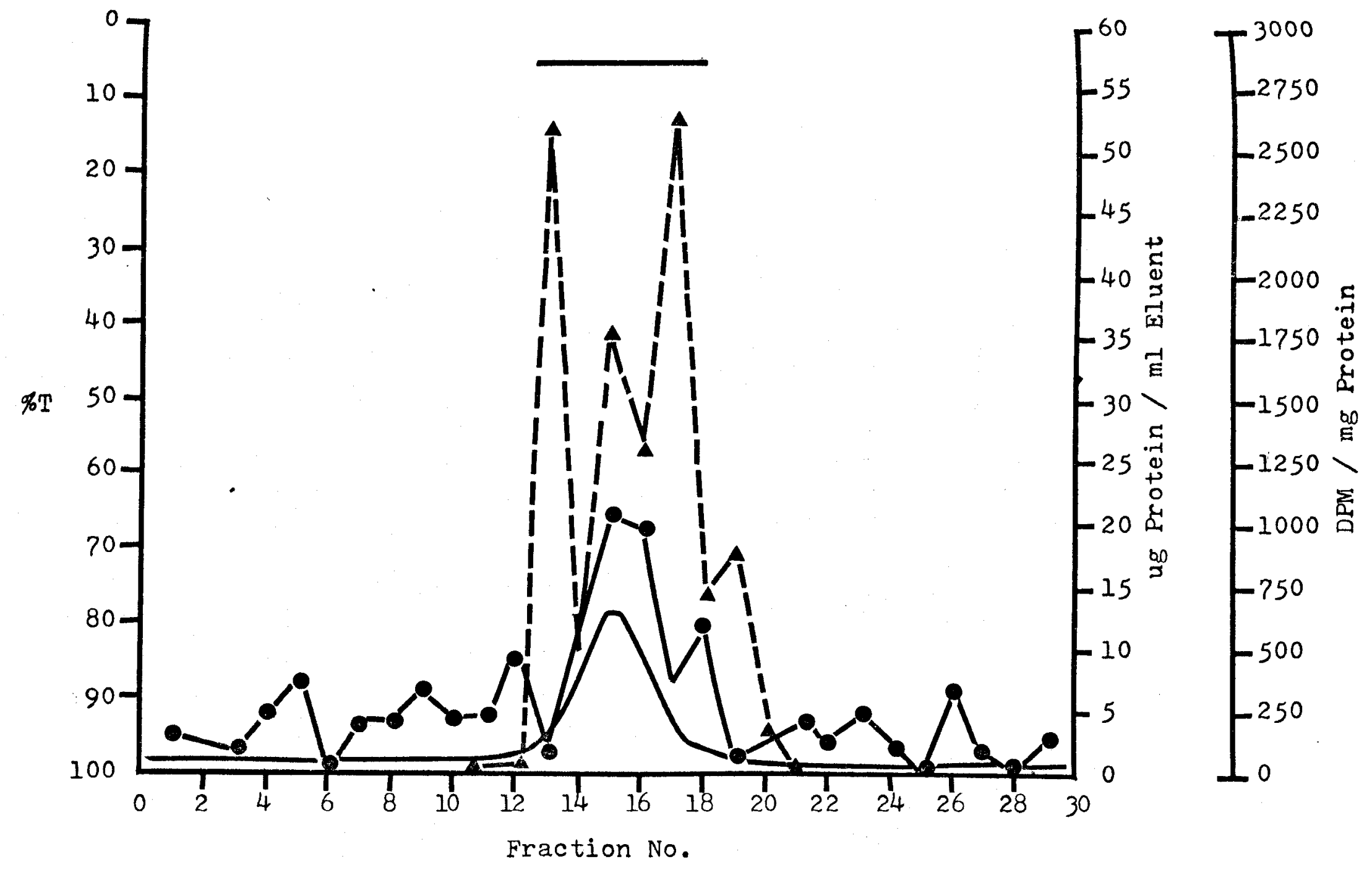

Figure 16. Protein content (-O), radioactivity (A- $-\mathbf{\Delta})$, and elution pattern (-) of lyophilized nuclear preparation from a physiological calcium concentration incubation medium passed through a column of Sephadex G-100. Flow rate $-35 \mathrm{ml} / \mathrm{hr}, 4 \mathrm{ml}$ fractions collected. Fractions assayed for hypocalcemic activity ( $)$. Two times the expansion of the $\% \mathrm{~T}$ scale. 
2300 DPM per mg protein for the microsomal preparation (Figure 14).

Sorgente (1969) demonstrated that a calcium concentration of $45 \mathrm{mg} / 100 \mathrm{ml}$, a concentration four times greater than a physiological calcium level, enhanced the biosynthesis and release of CT from thyroid slices in biosynthetic media. However, he did not determine the influence of a low calcium concentration on hormone biosynthesis and release. Therefore, microsomal, mitochondrial, and nuclear preparations were obtained from an incubation medium of Krebs-Ringer bicarbonate buffer containing a calcium concentration of $4.8 \mathrm{mg} / 100 \mathrm{ml}$. This low level of calcium in the incubation medium was selected due to its approximation to the physiological minimum to which serum calcium could decline as occurs in parathyroidectomized animals or in animals on a severely restricted calcium diet. A change in the extent of tracer amino acid incorporation or an alteration in the hypocalcemic response of the three subcellular fractions at this low calcium concentration might give some indication of the degree of sensitivity of the hormone biosynthetic process to short-term deprivation of calcium. The influence of a low calcium concentration on hormone biosynthesis and incorporation of glycine-C-14 is presented in Table XIV. The microsomal fraction decreased serum calcium by $12.4 \%$ and the change in calcium in $\mathrm{mg} \%$ was -0.034 per $10 \mu \mathrm{g}$ protein. These values are quite similar to those for the microsomal preparations obtained from a physiological calcium-containing medium (Table XIII) 


\section{TABLE XIV}

HYPOCALCEMIC ACTIVITY OF MICROSOMAL, MITOCHONDRIAL, AND NUCLEAR PREPARATIONS OBTAINED FROM INCUBATION MEDIA CONTAINING LOW

CALCIUM CONCENTRATIONS AND $2.5 \mu \mathrm{Ci}$ OF GLYCINE-C-I4 (UL)

\begin{tabular}{|c|c|c|c|c|c|c|c|}
\hline Preparation & $\begin{array}{c}\text { Animal } \\
\text { No. }\end{array}$ & $\frac{\mathrm{Ca}}{\mathrm{t}_{0}}$ & $\frac{\mathrm{mg} \%}{t_{50}}$ & $\begin{array}{r}\Delta \\
10\end{array}$ & $\begin{array}{l}\text { Ca mg\%/ } \\
\mu g \text { Protein }\end{array}$ & $\begin{array}{l}\text { Net } \\
\text { Change }\end{array}$ & $\stackrel{\%}{\%}$ \\
\hline \multirow[t]{12}{*}{ Microsomal } & 1 & 10.28 & 9.34 & & -0.022 & -0.94 & -9.1 \\
\hline & 2 & 9.72 & 9.72 & & 0.000 & 0.00 & 0.0 \\
\hline & 3 & 11.42 & 8.82 & & -0.066 & -2.60 & -22.8 \\
\hline & 4 & 9.51 & 9.61 & & +0.002 & +0.10 & +1.1 \\
\hline & 5 & 10.69 & 10.17 & & -0.012 & -0.52 & -4.9 \\
\hline & 6 & 11.48 & 8.87 & & -0.066 & -2.61 & -22.7 \\
\hline & 7 & 10.52 & 9.34 & & -0.028 & -1.18 & -11.2 \\
\hline & 8 & 11.33 & 9.03 & & -0.058 & $-2 \cdot 30$ & $-20 \cdot 3$ \\
\hline & 9 & 11.54 & 9.61 & & -0.049 & -1.93 & -16.7 \\
\hline & 10 & 10.87 & 8.92 & & -0.046 & -1.95 & -17.9 \\
\hline & 11 & 11.17 & 9.89 & & -0.031 & -1.28 & -11.5 \\
\hline & Mean & $\pm S . D$. & & & $-0.034 \pm 0$ & .024 & -12.4 \\
\hline \multirow[t]{8}{*}{ Mitochondrial } & 1 & 10.20 & 9.41 & & -0.022 & -0.79 & -7.7 \\
\hline & 2 & 9.46 & 9.41 & & -0.001 & -0.05 & -0.5 \\
\hline & 3 & 8.02 & 8.84 & & +0.023 & +0.82 & +10.2 \\
\hline & 4 & 8.74 & 8.35 & & -0.011 & -0.39 & -4.5 \\
\hline & 5 & 9.73 & 9.46 & & -0.008 & -0.27 & -2.8 \\
\hline & 6 & 9.25 & 8.89 & & -0.010 & -0.36 & -3.9 \\
\hline & 7 & 9.57 & 9.25 & & -0.009 & -0.32 & -3.3 \\
\hline & Mean & $\pm S . D$. & & & $-0.005 \pm 0$ & .014 & -1.8 \\
\hline \multirow[t]{8}{*}{ Nuclear } & 1 & 10.93 & 10.40 & & -0.022 & -0.53 & -4.8 \\
\hline & 2 & 9.89 & 10.06 & & +0.007 & +0.17 & +1.7 \\
\hline & 3 & 10.00 & 9.40 & & -0.025 & -0.60 & -6.0 \\
\hline & 4 & 10.57 & 10.98 & & +0.017 & +0.41 & +3.9 \\
\hline & 5 & 11.41 & 10.17 & & -0.051 & -1.24 & -10.9 \\
\hline & 6 & 10.00 & 10.63 & & +0.026 & +0.63 & +6.3 \\
\hline & 7 & 9.83 & 10.17 & & +0.014 & +0.34 & +3.5 \\
\hline & 8 & 10.51 & 9.94 & & -0.024 & -0.57 & -5.4 \\
\hline & Mean & ES.D. & & & $-0.007 \pm 0$ & .026 & -1.5 \\
\hline
\end{tabular}


in which the decrease in serum calcium was $11.5 \%$ and serum calcium was decreased by $0.024 \mathrm{mg} \% / 10 \mu \mathrm{g}$ protein. Unlike the microsomal preparation, injection of the mitochondrial and nuclear preparations elicited less than a $2 \%$ hypocalcemic response in rats. Microsomal preparations obtained from incubation media containing either low or physiological calcium concentrations both lowered serum calcium by approximately $12 \%$. Figure 17 shows the elution pattern, protein content, and radioactivity of a microsomal preparation obtained from an incubation medium containing $4.8 \mathrm{mg} \mathrm{Ca} /$ $100 \mathrm{ml}$ of Krebs-Ringer bicarbonate buffer. The elution, protein, and radioactivity peaks corresponded quite closely. Once again, the peak protein content of the microsomal fraction was quite high, approximating $360 \mu \mathrm{g}$ per $\mathrm{ml}$ of eluent. The radioactive peak of the purified microsomal preparation was approximately $5500 \mathrm{DPM} /$ mg protein. Since the hypocalcemic response of this microsomal preparation was almost identical to that of the microsomal preparation obtained from an incubation medium containing a physiological calcium concentration, it was apparent that glycine-C-14 was being incorporated at an enhanced rate, into microsomal proteins other than CT. Actually, the high value can not be readily explained, since one would expect the same or lower levels of incorporation of tracer amino acid into a microsomal preparation obtained from an incubation medium containing a low calcium concentration. 


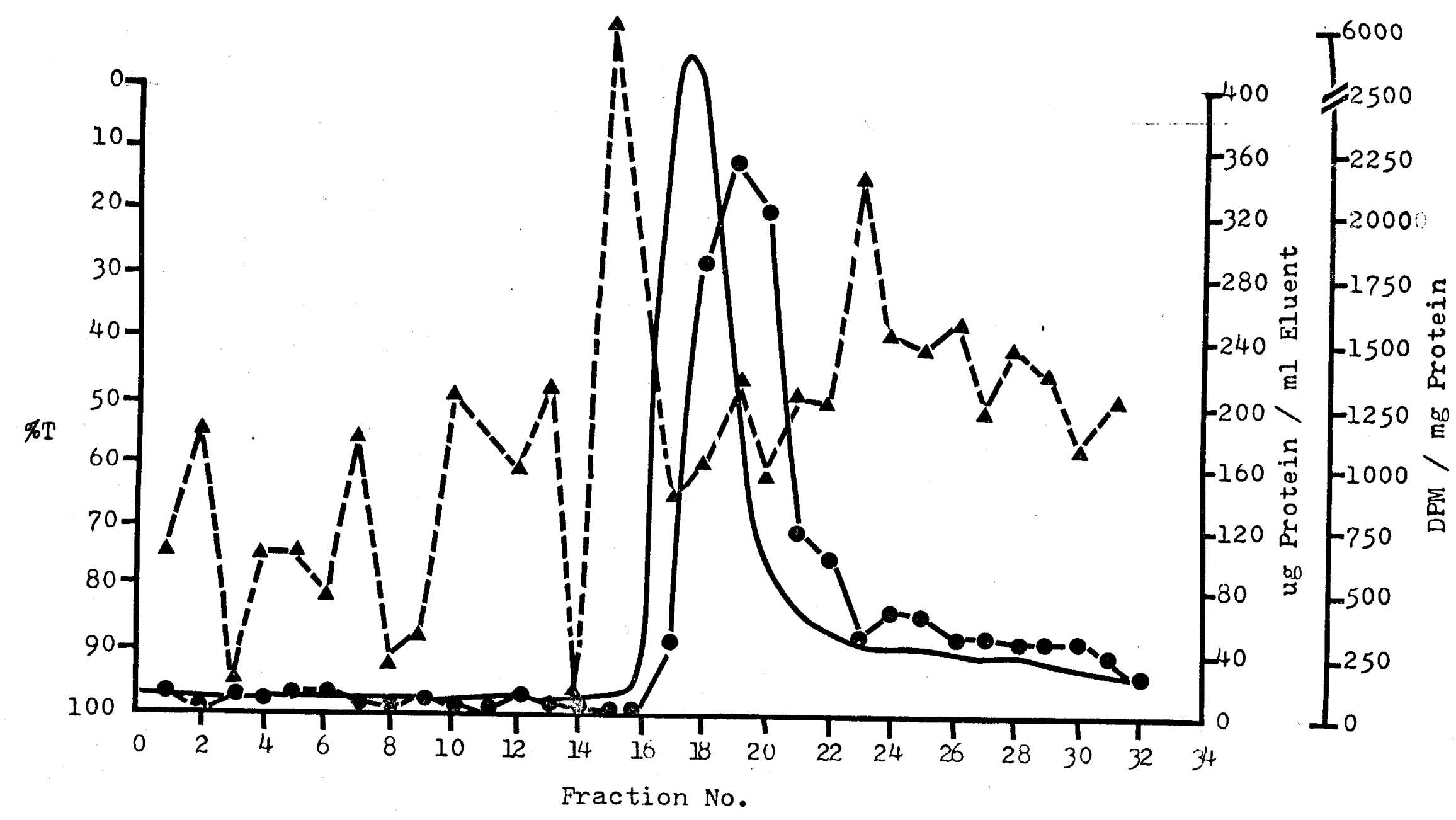

Figure 17 . Protein content $(\bullet-\bullet)$, radioactivity $(\Delta--\mathbf{- \Lambda})$, and elution pattern incubation medium passed throughal preparation from a low calcium concentration $4 \mathrm{ml}$ fractions collected through a column of Sephadex G-100. Flow rate $-35 \mathrm{ml} / \mathrm{hr}$

Two times the expansion of the \%T scale. 
The elution pattern, protein content, and radioactivity of a mitochondrial preparation obtained from an incubation medium containing a low calcium concentration are exhibited in Figure 18. The elution, protein and radioactivity peaks correspond quite closely. An incongruent feature is a rather large protein peak of approximately $300 \mu \mathrm{g} / \mathrm{ml}$ eluent compared to that observed for the mitochondrial preparation obtained from an incubation medium containing a physiological calcium concentration (Figure 15). However, the peak of radioactivity of approximately 3,000 DPM per mg protein is quite similar to that of mitochondrial and microsomal preparations obtained from incubation media containing low or physiological calcium concentrations.

Figure 19 shows the elution pattern, protein content, and radioactivity of purified nuclear preparation obtained from an incubation medium containing a low calcium concentration. Once again, there is a close correspondence between the elution, protein, and radioactivity peaks. The radioactivity peak corresponds to approximately 5500 DPM per $\mathrm{mg}$ protein. The high radioactivity peaks of approximately $5500 \mathrm{DPM} / \mathrm{mg}$ protein of the nuclear (Figure 19) and microsomal preparations (Figure 17) do not indicate a consistent trend of increased incorporation of radioactivity into protein of subcellular fractions obtained from media containing since the mitochondrial preparation had a radioactivity peak of only 3,000 DPM per mg protein (Figure 18). 


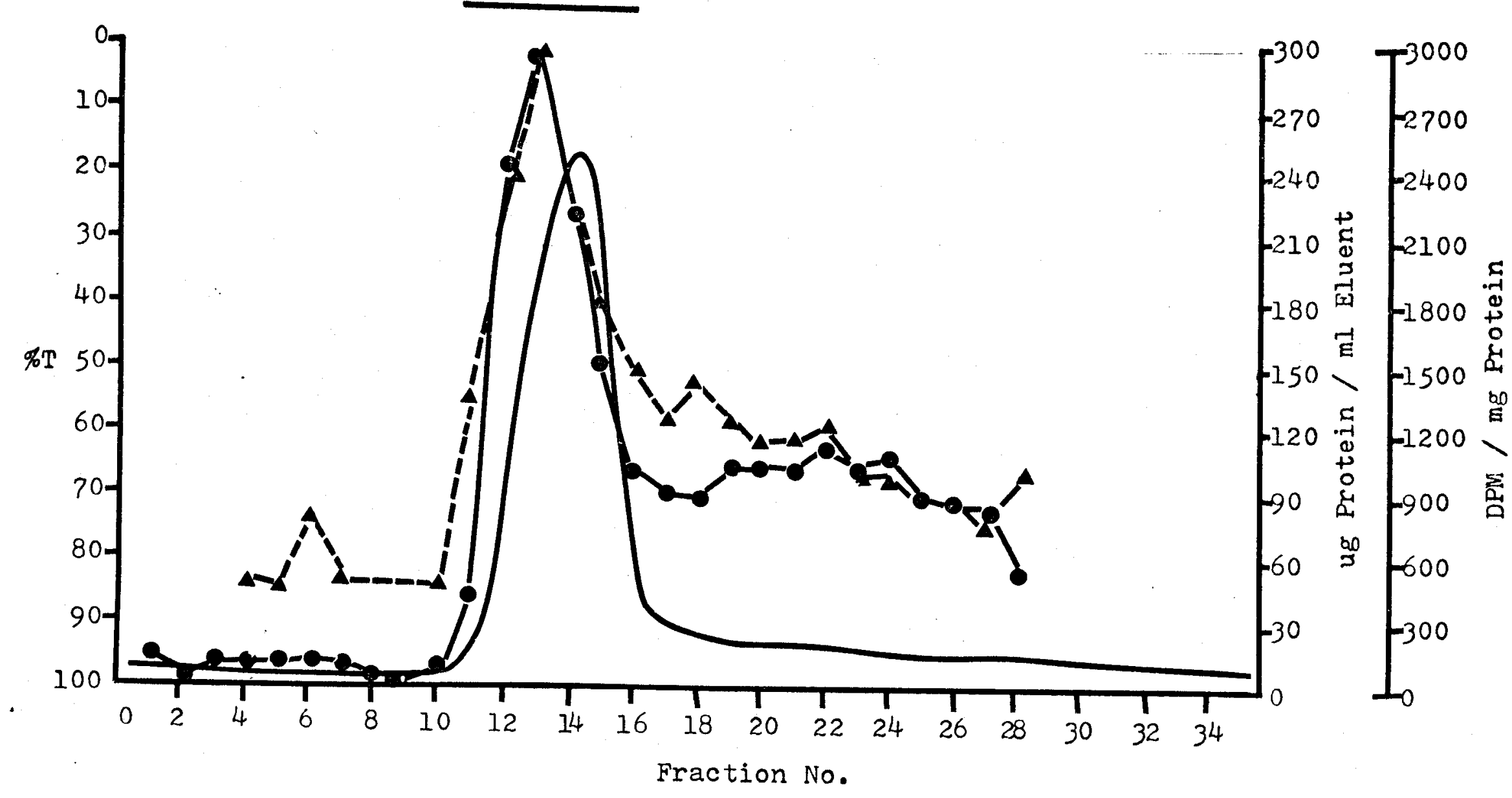

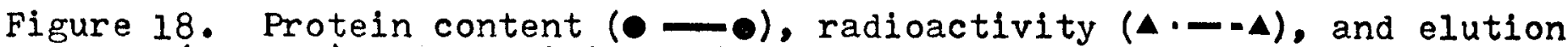
pattern (-) of lyophilized mitochondrial preparation from a low calcium concentration medium passed through a column of Sephadex G-100. Flow rate- $35 \mathrm{ml} / \mathrm{hr} ; 4 \mathrm{ml}$ fractions collected. Fractions assayed for hypocalcemic activity (- Two times the expansion of the \% scale. 


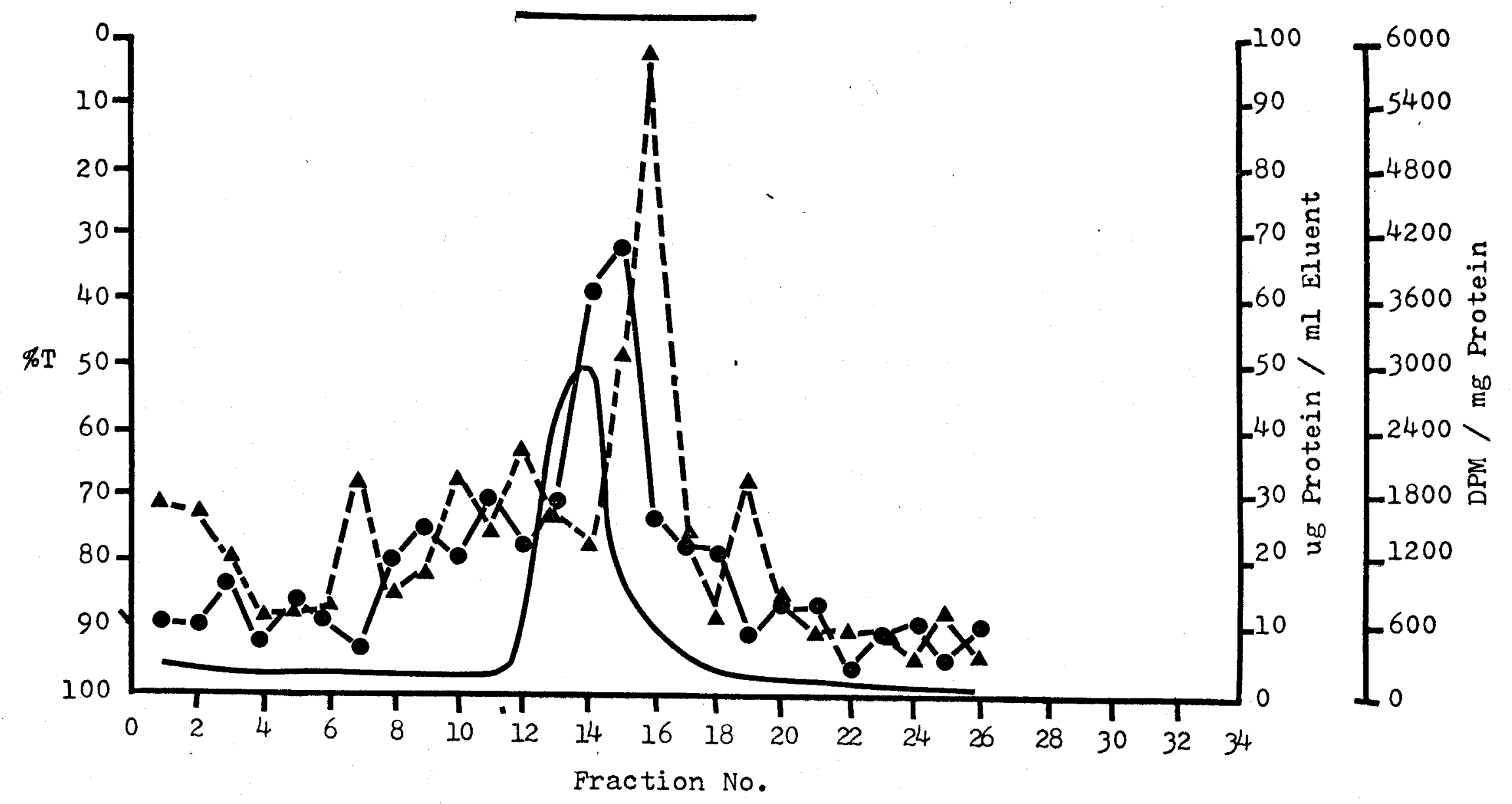

Figure 19. Protein content (O-O), radioactivity ( $\mathbf{-}-\mathbf{-}-\mathbf{A})$, and elution pattern ( ) of lyophilized nuclear preparation from a low calcium concentration incubation medium passed through a column of Sephadex G-100. Flow rate $-35 \mathrm{ml} / \mathrm{hr}$; $4 \mathrm{ml}$ fractions collected. Fractions assayed for hypocalcemic activity ( 2 Two times the expansion of the \% scale. 
The hypocalcemic response of subcellular fractions incubated in media containing a high calcium concentration is presented in Table XV. The mitochondrial and nuclear preparations were slightly hypercalcemic, increasing serum calcium by 6.4 and $6.3 \%$, respectively. But the microsomal fraction was extremely hypocalcemic, decreasing serum calcium by $22.5 \%$ and changing serum calcium by $-0.083 \mathrm{mg} \%$ per $10 \mu \mathrm{g}$ protein. This clearly indicated that a high calcium concentration in the incubation medium had stimulated calcitonin biosynthesis in the microsomal fraction. The $22.5 \%$ decrease in serum calcium was almost twice that observed with microsomal preparations obtained from incubation media containing physiological calcium concentration ( $11.5 \%$, Table XIII) and low (12.4\%, Table XIV) calcium concentrations. Moreover, the change in serum calcium of $-0.085 \mathrm{mg}$ per $10 \mu \mathrm{g}$ protein was over twice that observed from microsomes obtained from an incubation medium containing a physiological calcium concentration (which changed serum calcium by $-0.024 \mathrm{mg} \%$ per $10 \mu \mathrm{g}$ protein) and those obtained from a medium containing a low calcium concentration (which changed serum calcium by $-0.034 \mathrm{mg} \%$ per $10 \mu \mathrm{g}$ protein). The elution pattern, protein content, and radioactivity of the microsomal preparation obtained from an incubation medium containing a high calcium concentration is shown in Figure 20. The protein peak corresponded to $400 \mu \mathrm{g}$ protein per $\mathrm{m} 1$ eluent. The peak of radioactivity was $900 \mathrm{DPM} / \mathrm{mg}$ protein and occurred several fractions beyond the 
TABLE XV

HYPOCALCEMIC ACTIVITY OF MICROSOMAL, MITOCHONDRIAI, AND NUCLEAR PREPARATIONS OBTAINED FROM INCUBATION MEDIA CONTAINING HIGH CALCIUM CONCENTRATIONS AND $2.5 \mu \mathrm{Ci}$ OF GLYCINE-C-I4 (UL)

\begin{tabular}{|c|c|c|c|c|c|c|c|}
\hline Preparation & $\begin{array}{l}\text { Animal } \\
\text { No. }\end{array}$ & $\frac{\mathrm{Ca}}{\mathrm{t}_{0}}$ & $\frac{m g \%}{t_{50}}$ & $\begin{array}{r}\Delta \\
10\end{array}$ & $\begin{array}{l}\text { Ca mg\%/ } \\
\mu g \text { Protein }\end{array}$ & $\begin{array}{l}\text { Net } \\
\text { Change }\end{array}$ & $\begin{array}{c}\% \\
\text { Change }\end{array}$ \\
\hline \multirow{8}{*}{ Microsomal } & 1 & 11.16 & 9.03 & & -0.049 & -2.13 & $-19 \cdot 1$ \\
\hline & 2 & 11.48 & 9.08 & & -0.055 & -2.40 & $-20 \cdot 9$ \\
\hline & 3 & 11.10 & 8.67 & & -0.056 & -2.43 & -21.9 \\
\hline & 4 & 10.92 & 9.03 & & -0.044 & -1.89 & $-17 \cdot 3$ \\
\hline & 5 & 11.99 & 9.08 & & -0.067 & -2.91 & $-24 \cdot 3$ \\
\hline & 6 & 11.23 & 8.37 & & -0.066 & -2.86 & $-25 \cdot 5$ \\
\hline & 7 & 11.16 & 9.13 & & -0.047 & -2.03 & -18.2 \\
\hline & 8 & 11.40 & 8.41 & & -0.140 & -2.99 & -26.2 \\
\hline \multirow[t]{3}{*}{ r } & 9 & 11.74 & 8.56 & & -0.149 & -3.18 & -27.1 \\
\hline & 10 & 10.93 & 8.17 & & -0.129 & -2.76 & $-25 \cdot 3$ \\
\hline & 11 & 10.51 & 8.21 & & -0.107 & $-2 \cdot 30$ & -21.9 \\
\hline \multicolumn{4}{|c|}{ Mean \pm S. D. } & & $-0.083 \pm 0$ & .040 & -22.5 \\
\hline \multirow[t]{7}{*}{ Mitochondrial } & 1 & 8.74 & 9.14 & & +0.011 & +0.40 & +4.6 \\
\hline & 2 & 8.79 & 10.22 & & +0.039 & +1.43 & +16.3 \\
\hline & 3 & 8.99 & 9.20 & & +0.006 & +0.21 & $+2 \cdot 3$ \\
\hline & 4 & 8.55 & 9.10 & & +0.015 & +0.55 & +6.4 \\
\hline & 5 & 8.74 & 9.40 & & +0.018 & +0.66 & +7.6 \\
\hline & 6 & 9.46 & 9.56 & & +0.003 & +0.10 & +1.1 \\
\hline & Mean & \pm S. D. & & & $+0.015 \pm 0$ & .010 & +6.4 \\
\hline \multirow[t]{7}{*}{ Nuclear } & 1 & 9.35 & 10.00 & & +0.023 & +0.65 & +7.0 \\
\hline & 2 & 10.06 & 8.99 & & -0.038 & -1.07 & -10.6 \\
\hline & 3 & 9.10 & 10.57 & & +0.052 & +1.47 & +16.2 \\
\hline & 4 & 8.74 & 9.25 & & +0.018 & +0.51 & +5.8 \\
\hline & 5 & 9.25 & 10.86 & & +0.057 & +1.61 & +17.4 \\
\hline & 6 & 9.71 & 9.89 & & +0.006 & +0.18 & +1.9 \\
\hline & \multicolumn{3}{|c|}{ Mean $\pm S . D$. } & & $+0.020 \pm 0$ & .033 & +6.3 \\
\hline
\end{tabular}




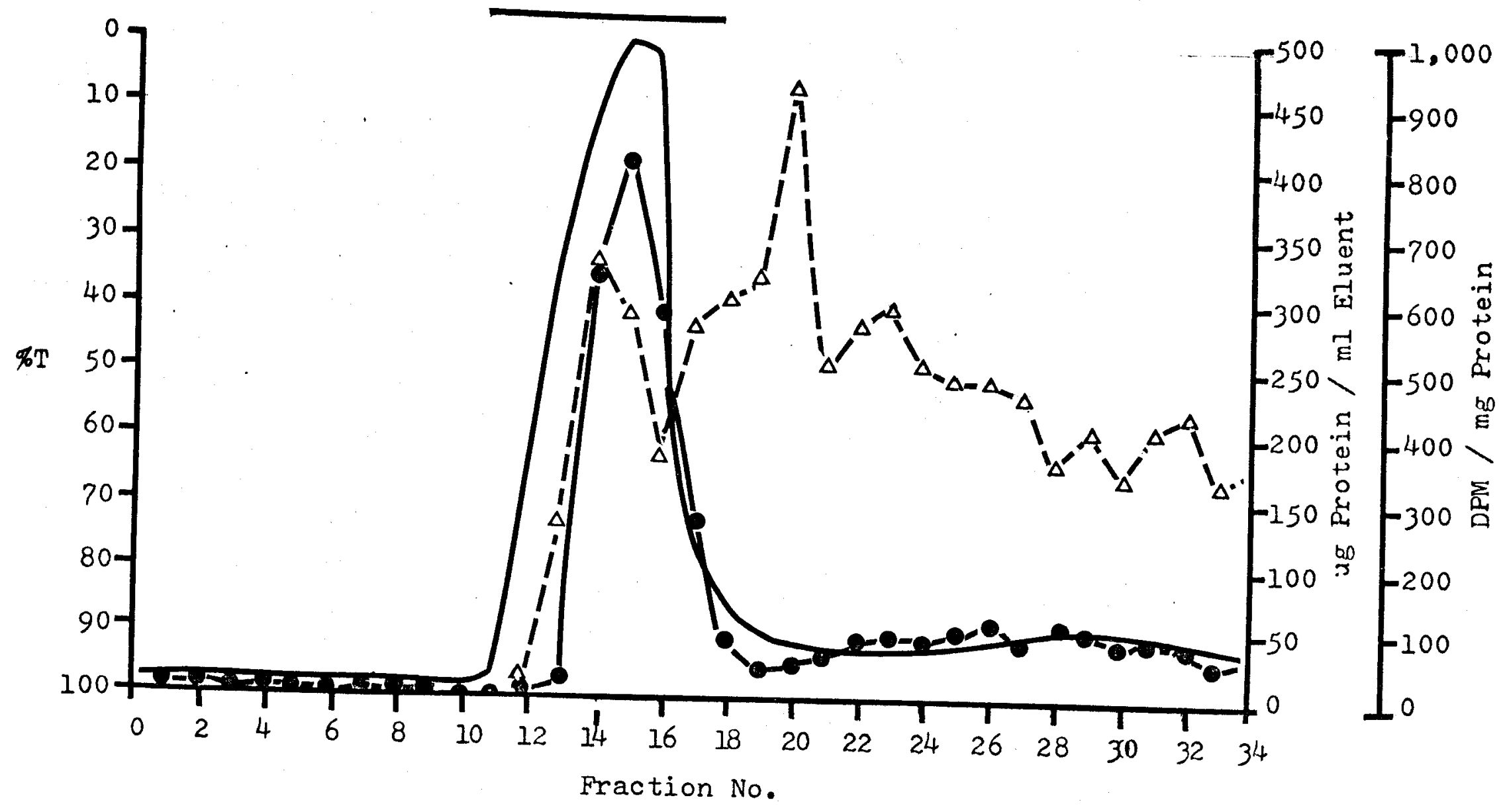

$\frac{1}{E}$

Figure 20. Protein content (- - ), radioactivity $(\Delta--\Delta)$, and elution pattern incubation medium passed through al preparation from a high calcium concentration $4 \mathrm{ml}$ fractions collected. Fractions column of Sephadex G-l00. Flow rate - $35 \mathrm{ml} / \mathrm{hr}$

Two times the expansion of the \% scale. 
elution and protein peaks. The relatively small amount of incorporation of glycine-c-14 into this fraction is not readily explained since one would expect increased incorporation of the label into a preparation undergoing increased protein biosynthesis. This low amount of radioactivity was not a consistent finding since Figure 21 shows that the mitochondrial preparation had 2500 DPN per $\mathrm{mg}$ protein. The elution and protein peaks corresponded quite closely.

The elution pattern, protein content, and radioactivity of a nuclear preparation which was obtained from an incubation medium containing a high calcium concentration is shom in Figure 22. In this particular experiment, the protein peak occurred far beyond the elution and radioactive peaks. The protein peak was rather small, representing $50 \mathrm{\mu g}$ of protein per $\mathrm{ml}$ eluent, and the radioactivity peak was approximately $6,000 \mathrm{DFM} / \mathrm{mg}$ protein. The foregoing study which monitored the incorporation of glycine-C-14 into subcellular fractions did not demonstrate a preferential incorporation of the tracer amino acid into the hypocalcemic, microsomal fraction. Moreover, the extent of incorparation of the label into subcellular preparations was not iniluenced by the calcium concentration of the biosynthetic media, since no consistent patterns in glycine-C-14 incorporation were evident. However, it was apparent that glycine-C-14 was being incorporated into all of the proteins of the microsomal, mitochondrial, and nuclear preparations without any preferential incorporation into CT of the microsomal preparation. 


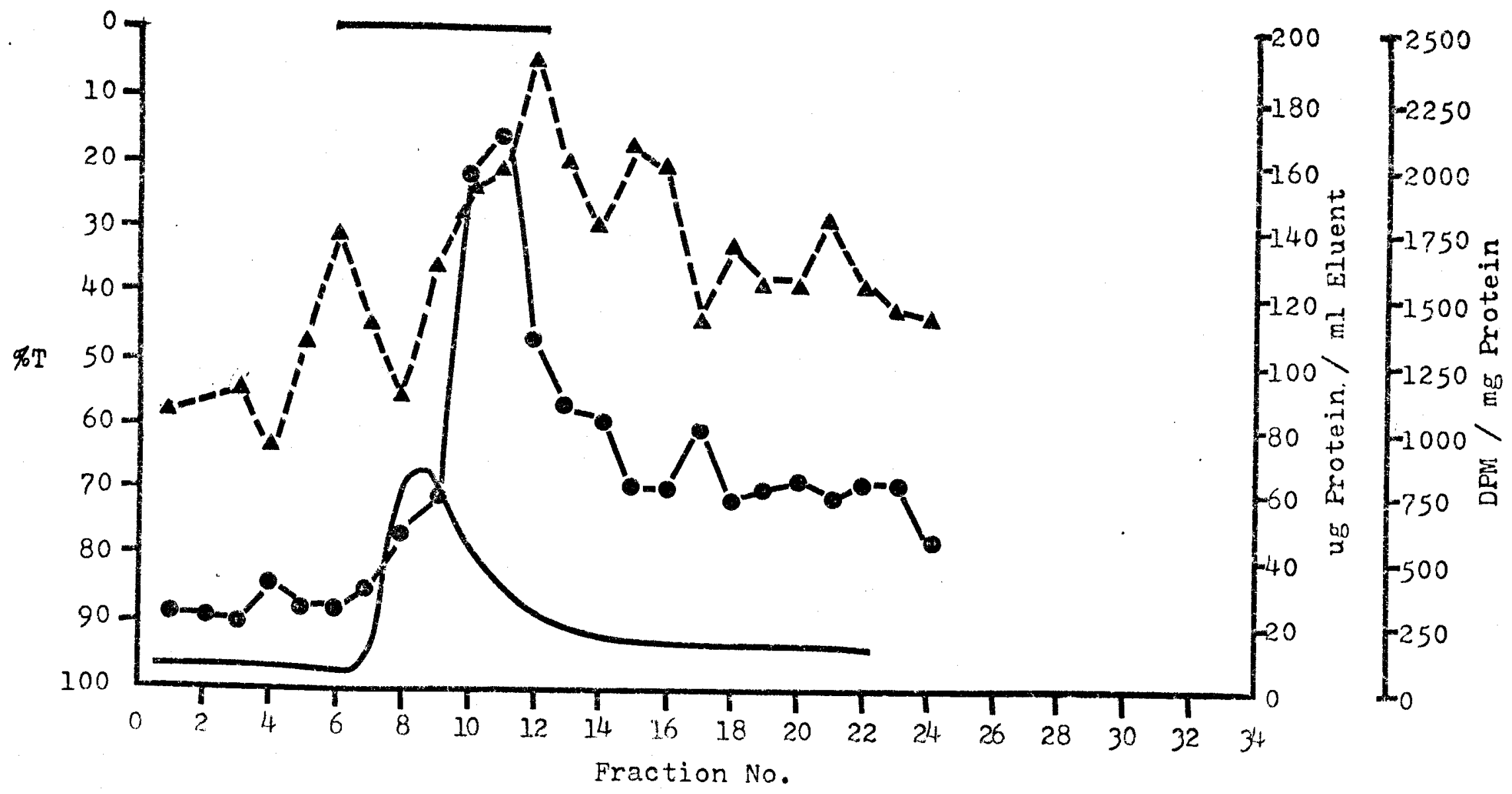

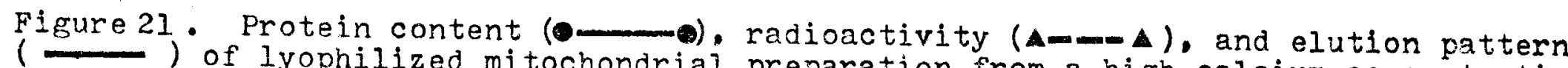
(ncubation medichopilized mitochondrial preparation from a high calcium concentration $4 \mathrm{ml}$ fractions collected through a column of Sephadex G-100. Flow rate - $35 \mathrm{ml} / \mathrm{hr}$ times the expansion of the \%r scale. assayed for hypocalcemic activity (- Two 


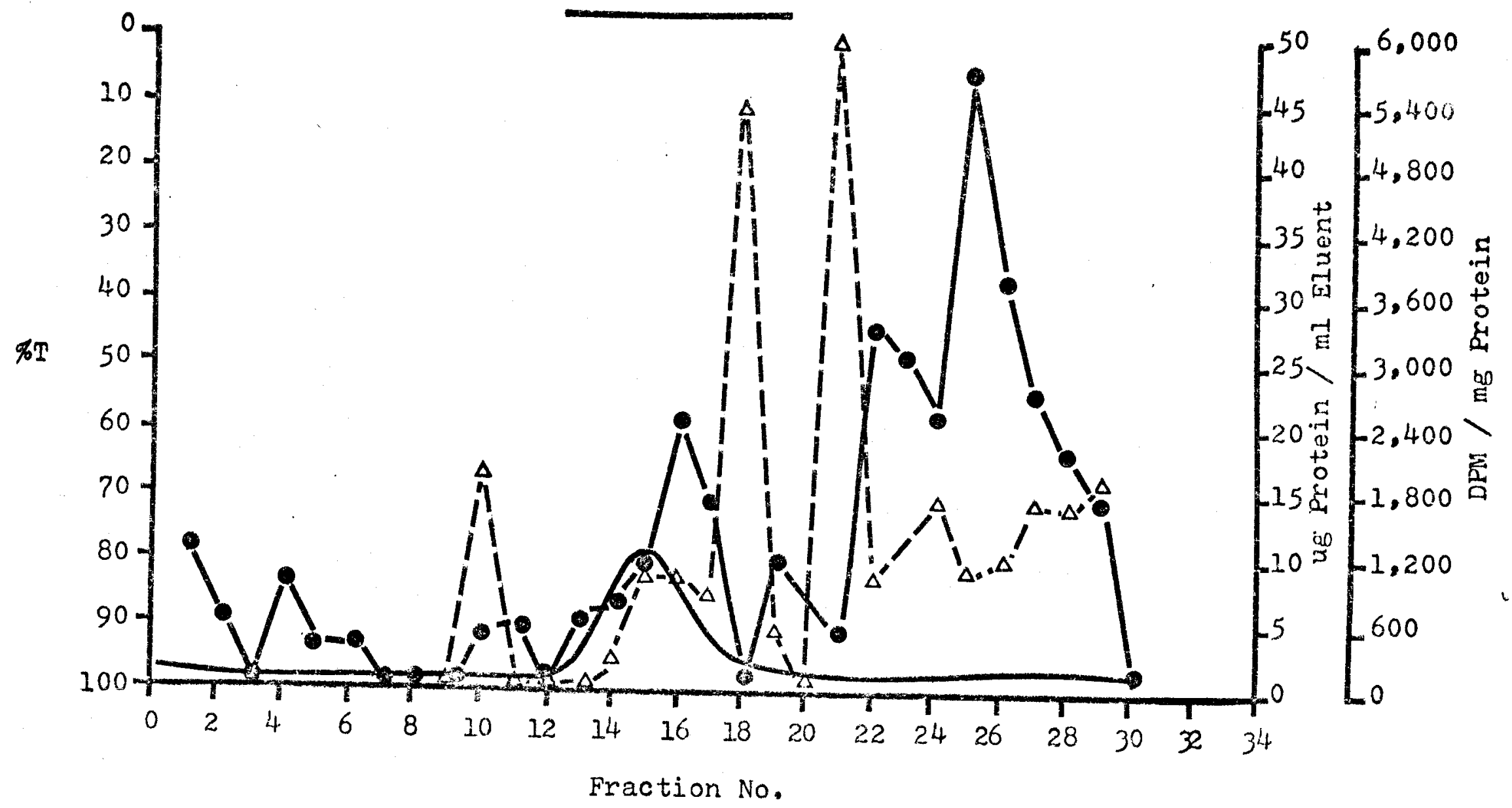

Figure 22. Protein content (- - radioactivity $(\Delta \cdot--\Delta)$, and elution pattern incubation medium of lyophilized nuclear preparation from a high calcium concentration $4 \mathrm{ml}$ fractions collected. through a column of Sephadex G-100. Flow rate - $35 \mathrm{ml} / \mathrm{hr}$ Two times the expansion of the $\%$ T scale. 
A statistical analysis was utilized to compare the hypocalcemic response of Sephadex G-100-purified and labeled mitochondrial, and nuclear preparations to that of the consistently hypocalcemic microsomal preparations. The analysis, employing an unpaired student's "T" Test, is presented in Table XVI. A significant difference in hypocalcemic activity was evidenced when the mitochondrial and nuclear preparations obtained from incubation media containing a physiological calcium concentration were compared to the hypocalcemic response of the microsomal preparation which was obtained from the same type of biosynthetic media. While the microsomal preparation decreased serum calcium by $0.024 \mathrm{mg} \%$ per $10 \mu \mathrm{g}$ protein, the mitochondrial preparation and nuslear preparation actually increased serum calcium by 0.007 and $0.045 \mathrm{mg} \% / 10 \mu \mathrm{g}$ protein, respectively. Similarly, the mitochondrial and nuclear preparations, obtained from incubation medium containing a low calcium concentration, were significantly different in their hypocalcemic responses from that of the microsomal preparation obtained from this biosynthetic medium. The microsomal fraction decreased serum calcium by $0.034 \mathrm{mg} / 10 \mu \mathrm{g}$ protein while the mitochondrial fraction decreased serum calcium by only $0.005 \mathrm{mg} \% / 10 \mu \mathrm{g}$ protein and the nuclear preparation by $0.007 \mathrm{mg} \% / 10 \mathrm{ug}$ protein. Moreover, a highly significant difference in hypocalcemic activity was noted when the hypocalcemic response of the microsomal preparation obtained from a medium containing high calcium concentration was compared to thet of mitochondrial and nuclear preparations obtained from the same incubation medium. 
TABLE XVI

HYPOCALCEMIC ACTIVITY OF PURIFIED MICROSOMAI, WITOCHONDRIAI, AND NUCLEAR FREPARATIONS OBTAINED FROM INCUBATION MEDIA CONTAINING IOW, PHYSIOLOGICAL, AND HIGH CALCIUM CONCENTRATIONS

Preparation

$\triangle \mathrm{Ca} \mathrm{mg} \% /$

$10 \mu g$ Protein

क

Change

Pis

\section{Physiological}

Microsomal

Mitochonärial

Nuclear

$$
\begin{array}{lll}
-0.024 \pm 0.017 & -11.5 \\
+0.007 \pm 0.005 & +1.0 \\
+0.045 \pm 0.042 & +5.3
\end{array}
$$$$
0.001
$$$$
0.001
$$

\section{Low}

Microsomal

Mitochondrial

Nuclear

$$
\begin{aligned}
& -0.034 \pm 0.024 \\
& -0.005 \pm 0.014 \\
& -0.007 \pm 0.026
\end{aligned}
$$$$
-12.4
$$$$
-1.8
$$$$
0.01
$$$$
-1.5
$$$$
0.05
$$

HiEh

hicrosomal

Mitochondrial

Nuclear

$$
\begin{aligned}
& -0.083 \pm 0.040 \\
& +0.015 \pm 0.010 \\
& +0.020 \pm 0.033
\end{aligned}
$$$$
-22.5
$$$$
+6.4
$$$$
+6.3
$$$$
0.001
$$$$
0.001 \text {. }
$$

* Probebility of the significance of the difference of the means as determined by the use of an unpaired Student's "t" Test. 
The influence of varying calcium concentrations on the hypocalcemic response of microsomal preparations is shown in Table XVII. In this instance, the hypocalcemic response of Sephadex G-100 purified microsomal preparations obtained from incubation media of low and high calcium concentrations was compared to that of a microsomal preparation obtained from an incubation medium containing a physiological calcium concentration. It is quite apparent that lowering the calcium concentration of the biosynthetic media $(4.8 \mathrm{mg} / 100 \mathrm{ml})$ does not significantly alter the hypocalcemic response of the microsomal preparation when compared to that of a microsomal preparation obtained from a medium containing a physiological calcium concentration. However, increasing the calcium concentration of the biosynthetic medium to $26 \mathrm{mg}$ per $100 \mathrm{ml}$ buffer results in a very significant increase in the hypocalcemic response of the microsomal preparation. This clearly demonstrates that a high calcium concentration in the incubation medium enhances CT biosynthesis at the subcellular level, even though incorporation of glycine-C-14 into the microsomal preparation is not enhanced by this calcium concentration.

CHARACTERIZATION OF MICROSOMAL AND STANDARD CALCITONIN PREPARATIONS BY POLYACRYLAMIDE GEL ELECTROPHORESIS

The second part of this investigation dealt with establishing the identity ${ }_{i} i$ the hypocalcemic microsumal fraction by comparison with a standard calcitonin preparation of known potency. To accomplish this, microsomal and standard calcitonin preparations of 
TABLE XVII

THE INFIUENCE OF VARYING CAICIUM CONCENTRATIONS ON THE HYPOCALCEMIC RESPONSE OF MICROSOMAL PREFARATIOINS

\begin{tabular}{|c|c|c|c|c|c|}
\hline Preparation & $\underset{10}{\Delta} \stackrel{\mathrm{Ca}}{\mathrm{Aa}}$ & $\begin{array}{l}\mathrm{mg} \\
\operatorname{Prc}\end{array}$ & tein & Change & $P^{*}$ \\
\hline Physjological & -0.024 & \pm & 0.017 & -11.5 & \\
\hline Low & -0.034 & \pm & 0.024 & -12.4 & NS \\
\hline High & -0.083 & \pm & 0.040 & $-22 \cdot 5$ & 0.005 \\
\hline
\end{tabular}

* Probability of the significance of the difference of the means as deternired by the use of an unpaired Student's "t" Test. 
known potency were electrophoretically fractionated in polyacrylamide gels. Microsomal fractions were then assayed for homogeneity in polyacrylamide gels after purification through sephadex $G-100$ and $G-50$.

Initial experiments consisted in the electrophoretic resolution in polyacrylamide gels of a standard calcitonin preparation having a potency of $88 \mathrm{MRC}$ units/mg. A cationic polyacrylamide gel system was used. It was determined that $200 \mu \mathrm{g}$ of this standard CT preparation per $200 \mu l$ of sample gei solution were sufficient for resolving this calcitonin prevaration into its component peptide fractions after an electrophoretic running time of one hour and twenty minutes with a current of $2 \frac{1}{2}$ ma per gei column. The densitometer trace of this cationic standard gel is presented in Figure 23. Readily apparent from the densitometer trace are two well-defined protein bands in these gels. The resolved band nearest the end of the separation gel was the largest and most intensely stained. Virtually all of the standard CT sample had electrophoretically migrated from the sample gel into the separation gel.

A number of attempts were then made to resolve the Sephadex G-100 purified microsomal preparation in this cationic polyacrylamide gel system as was achieved with the standard preparation. Initial attempts to resolve this preparation were performed at $200 \mu \mathrm{gg}$ of microsomal sample per gel. Electrophoresis was carried out for the same length of time and milliamperage per gel as had proved successful in resolving the standard calcitonin preparation. However, none of the sample material electrophoretically migrated 


\section{End of \\ Separation \\ Gel}

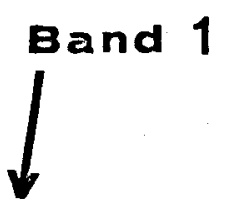

$\%$
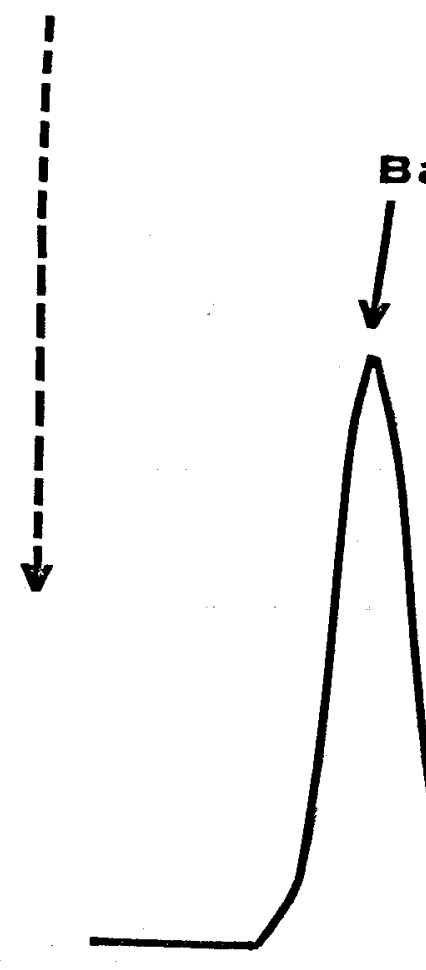

Band 2

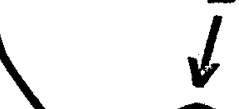

$\gamma^{2}$

Figure 23. Densitometer trace of a stained polyacrylamide gel. Two hundred micrograms of an $88 \mathrm{MRC}$ unit calcitonin standard were resolved in a cationic gel system. 
into the separation gel. In repetitive attempts to resolve this microsomal preparation, the sample concentration was increased to $1,500 \mu \mathrm{g}$ per gel. These rather high sample concentrations were utilized since it was apparent that a large portion of the microsomal preparation was not dissolved in the sample gel solution. This insoluble material was probably the remnants of the membra-nous macromolecular material of the microsomal fraction which was impossible to solubilize in the sample gel. Once again, all attempts to resolve the microsomal preparation in a cationic gel proved to be unsuccessful. Since the microsomal preparation could not be rescived in cationic gels as was the standard CT preparation, this cationic gel system could not be utilized to establish the identity of the hypocalcemic microsomal material by comparison with a standard CT preparation. Therefore, attempts were made to electrophoretically resolve the standard calcj.tonin and microsomal preparations into their component protein fractions in an anionic gel system. A standard CT preparation at a concentration of 333 $\mu \mathrm{g}$ per gel column was electrophoretically fractionated in an anionic gel at a current of $2 \frac{1}{2} \mathrm{ma}$ per column for one hour and forty minutes. Brompinenol blue served as the tracking dye in this anionic gel system. The trial run proved to be successful. The standard calcitonin preparation could be resolved into its component protein bands in both cationic and anionic polyacrylamide gel systems. This indicated the amphoteric nature of the standard CT preparation. However, not all of the standard CT preparation was resolved in the anionic gel system as evidenced by the substantial amount of sample remaining unresolved in the sample. 
A densitometer trace of a resolved $333 \mu \mathrm{g}$ calcitonin standard preparation is shown in Figure 24. An unusual finding was that in the anionic gel system, three major resolved protein bands (bands 1, 2 and 3) were evident as compared to the two bands observed in the cationic gels (Figure 23). There was also a minor, rather indistinct band at the beginning of the separation gel and a diffuse band a short distance beyond this minor band. It was possible that the major band (band 1 ) and the band adjoining it (band 3), which were observed at the end of the separation gel, represented a better resolution of the single broad band which was observed at the end of the cationic standard CT gel (Figure 23).

Initial electrophoretic separations of Sephadex G-1.00 microsomal preparations at sample concentrations varying from 200 to 1,500 pg at a constant current of $2 \frac{1}{2}$ ma per gel column and one hour and forty winutes electrophoretic separation time indjcated that the mierosomal preparation could, indeed, be resolved in this anionic gel system. Good resolution of protein bands was achieved at a sample concentration of $1,500 \mu \mathrm{g}$. Thus, this concentration was routinely used for electrophoretic resolution. A densitometer trace of the Sephadex G-100 microsomal preparation is shown in Figure 24. The three protein bands at the end of the separation gel consisting of two prominent bands (band 1 and 2) and one band (band 3) nexit to the dense bottom band were in identical positions to those of the 333 ug CT standard. An additional small band was evident a short distance from the end of the stacking gel which corresponded to the minor band in the densitometer trace of the CP standard. A densely stained band occurred at the interface of the 


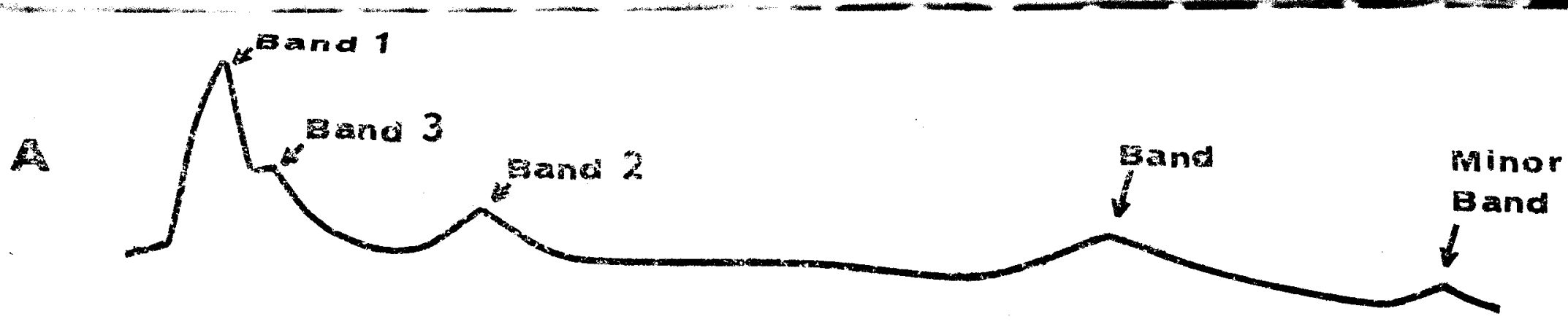

$B$
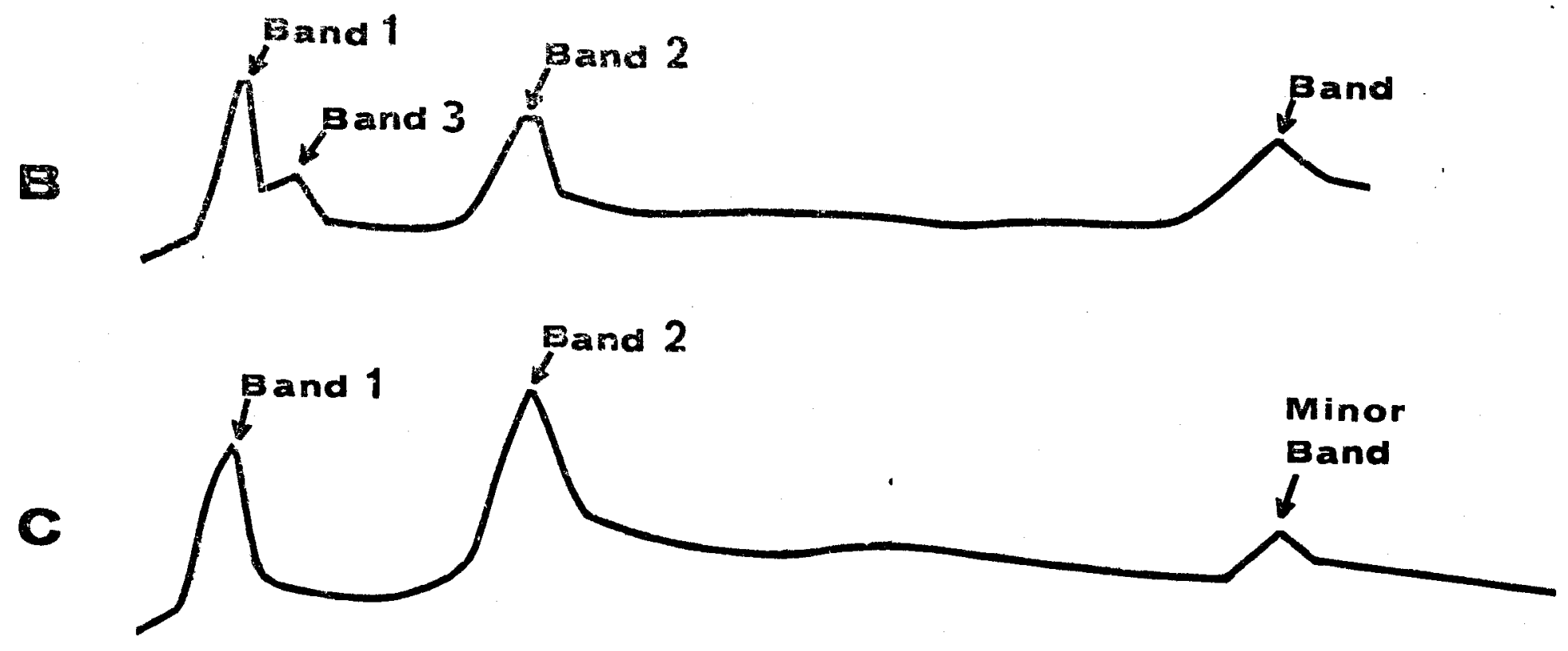

Figure 240 Densitometer traces of electrophoretically resolved hormone preparations in anionic polyacrylamide gels. $\mathrm{A}$, an 88 MRC unit calcitonin standard at a concentration of $333 \mu \mathrm{g}$; , Sephadex G-100 microsomal preparation at a concentration of $1,500 \mu \mathrm{g} ; \mathrm{C}$, Sephadex G-50 microsomal preparation at a concentration of $1,500 \mu \mathrm{g}$. 
separation and stacking gels although it is rot shown in the der.sitometer trace. This band may be attributable to macromolecular material such as the remnants of the membranous portion of the microsomai fraction. Such densely stained bands at the stacking gel/separation gel interface are routinely observed when serum proteins are fractionated in polyacrylamide gels.

A Sephadex G-50 purified microsomal preparation was run at the same sample concentration and milliamperage per gel as that for the Sephadex G-100 purified preparation. A densitometer trace of this preparation is presented in Figure 24. The two dense bands (band 1 and 2 ) at the end of the separation gel still remained and were identical in position to the stendard calcitonin preparation. However, the protein band (band 3) seen next to the dense band in the densitometer trace of the Sephadex $G-100$ gel and the anionic stariard CT preparation was no longer evident. There was a very minor protein band a short distance from the end of the stacking gel. Once again, a dense band remained at the separation gel/stacking gel interface but is not shown on the densitometer trace.

An electrophoretic separation was performed with the CT standard, the Sephadex G-100, and the Sephadex G-50 microsomal preparations in the same electrophoretic unit, thereby subjecting these three preparations to identical conditions of milliamperage per gel, electrophoretic separation time, and gel concentration. This was done to verify that the two major protein bands observed with these preparations were indeed attributabit to the same peptide fraction in the CT standard, the Sephadex G-1.00, and the Sephadex 
G-50 preparations. In all instances, the two major resolved protein bands of the standard CT and microsomal preparations corresponded well as seen in Figure 25. It is also apparent that the Sephadex G-50 purification step of the microsomal preparation had removed a peptide band (band 3) which was evident in the standard CT preparation and the Sephadex G-100 preparation (Figure 24 and 25).

CHARACTEP IZATION OF MICROSOMAL AND STANDARD CALCITONIN FREPARATICIS

\section{BY ISOELECTRIC FOCUSING}

Further characterization of the standard calcitonin preparaw tion and microsomal calcitonin preparations was accomplished by isoelectric focusing. This highly sensitive, resolving technique was utilized to determine a physical constant, the isoelectric point, of both standard and microsomal calcitonin preparations.

Seven milligrams of a standard CT preparation having a potency of 88 MRC units were electrofocused in an LKB 8100 Electrofocusing Column in a pH gradient extending from $\mathrm{pH} 3$ to 6 . Electrophoretic resolution of the standard CT preparation was completed after 48 houra. The electrofocusing column was then siowly emptied starting from the anode electrode solution and the elution pattern at $280 \mathrm{~nm}$ was monitored. Fractions of forty drops each were collected and the $\mathrm{pH}$ of these fractions was determined at $4^{\circ} \mathrm{C}$. This permitted the drawing of a pH curve for the entire electrcfocusing column. The elution pattern and the ph curve for the standard CI preparation are shown in Figure 26. The standard 


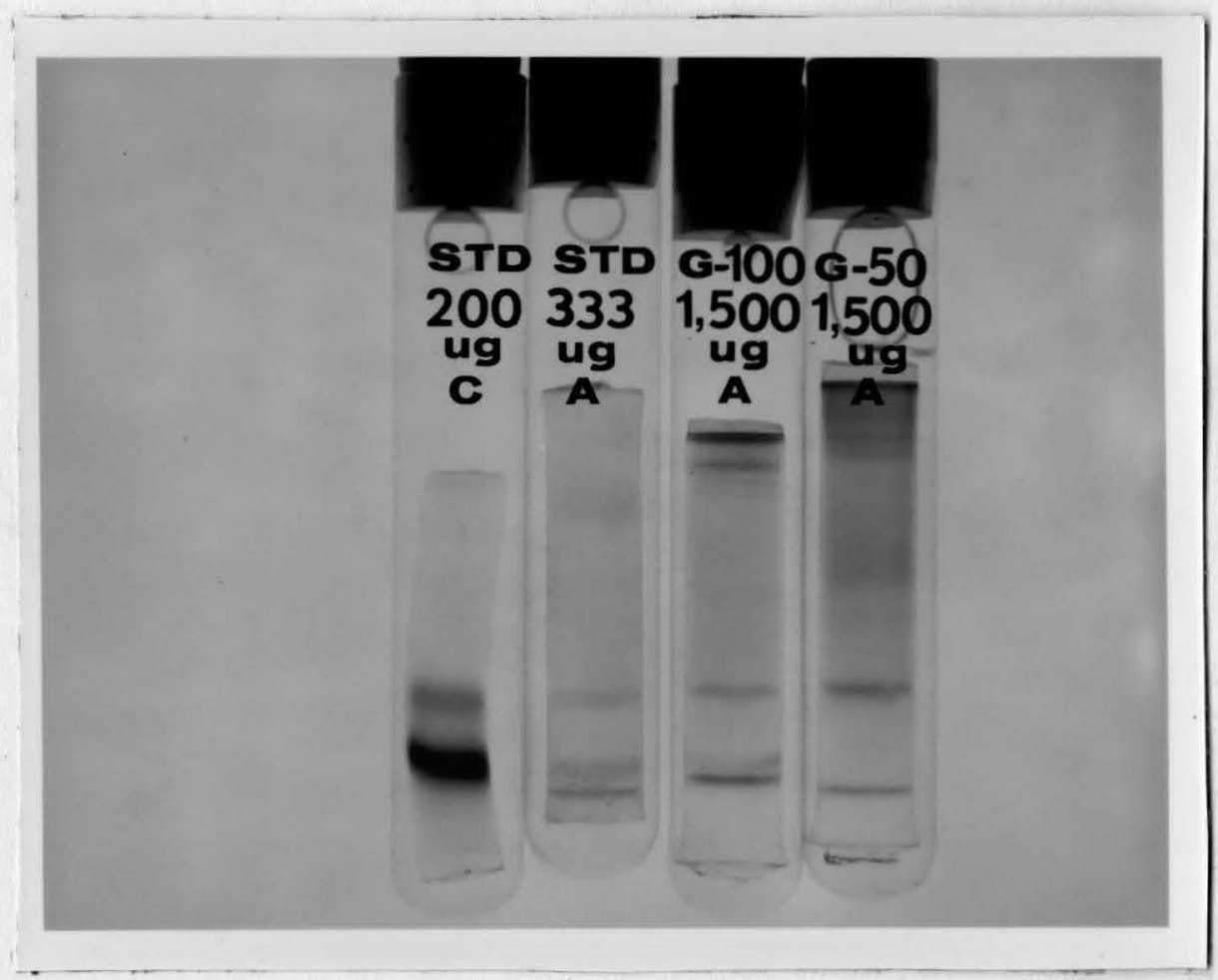

Figure 25. Polyacrylamide gels representing electrophoretically resolved standard and microsomal calcitonin preparations. The calcitonin standard at a concentration of $333 \mu \mathrm{g}$ and the Sephadex $G-100$ and $G-50$ purified microsomal preparations were fractionated in anionic gels under identical conditions. The standard calcitonin preparation at a concentration of $200 \mu \mathrm{g}$ was resolved in an anionic gel system and is shown for comparison. 


\section{p.1 4.50}

4.35

$4 \cdot 05$

3.90 Anode Electrode

$3 \cdot 75$

Solugion

$3 \cdot 60$

$3 \cdot 45$

$3 \cdot 30$

$3 \cdot 15$

. $3=00$

$28 \quad 40$

52

$60 \mathrm{ml}$

Figure 26. Separation of $7 \mathrm{mg}$ of a standard calcitonin preperation having a potency of 88 MRC units by the LS3 8100 Electrofocusing column In a $\mathrm{pH}$ gradient extending from pil 3 to 60 . The curve with two peaks shows the absorption of eluate at $280 \mathrm{~nm}$. The gteadily increasing curve is a plot of the ph gradient superimposed. 
CI prepaxation was resolved into two protein bands. The pI vaiues of these two resolved protein bands were determined from the plot of the steadily increasing pH curve. The resolved protein band, represented by the elution peak nearest the anode electrode solution had a $\mathrm{nl}$ of 3.70 while the pI of the second resolved protein band was 3.94 .

A similar type of determination was carried out with a Sephadex $G-50$ purified microsomal preparation which was obtained from an'incubation medium containing a physiological calcium concentration. Kowever, in this instance, the microsomal material was resolved in a pH gradient extending from $\mathrm{pH} 3.0$ to 5.0. The narrower pH gradient was utilized since a preliminary electrophoretic resolution of microsomal material in a broad pil gradient extending fror $\mathrm{pH} 3.0$ to 10.0 indicated that the $\mathrm{pI}$ of tine protein(s) of the microsomal material was approximately 4.0. Figure 27 shoms the electrophoretic resolution of $14 \mathrm{mg}$ of microsomal material in the IKB B100 Electrofocusing Column. Electrofocusing was carried out for 48 hours. The elution pattern was monitored at $280 \mathrm{~nm}$ and 40 drop fractions were collected. A pH curve for the entire electro. focusing column was determined at $4^{\circ} \mathrm{C}$. The microsomel preparation has resolvea lato two distinct protein banks having pI values of 3.49 and 3.84 these were determined from the plot of the steadim ily inereasing pil curve. These pI values for the microsomal $C T$ preparation vere quite similar to the pI values of 3.70 and 3.94 obtained for the two resolved protein bands of the standard CT preparation (Fjeure 26). It is possible that the pI values of the two preparations would have been more nearly the same had the 
PH 4.0

\section{$3 \cdot 9$}

$3 \cdot 8$

3.7 Anode

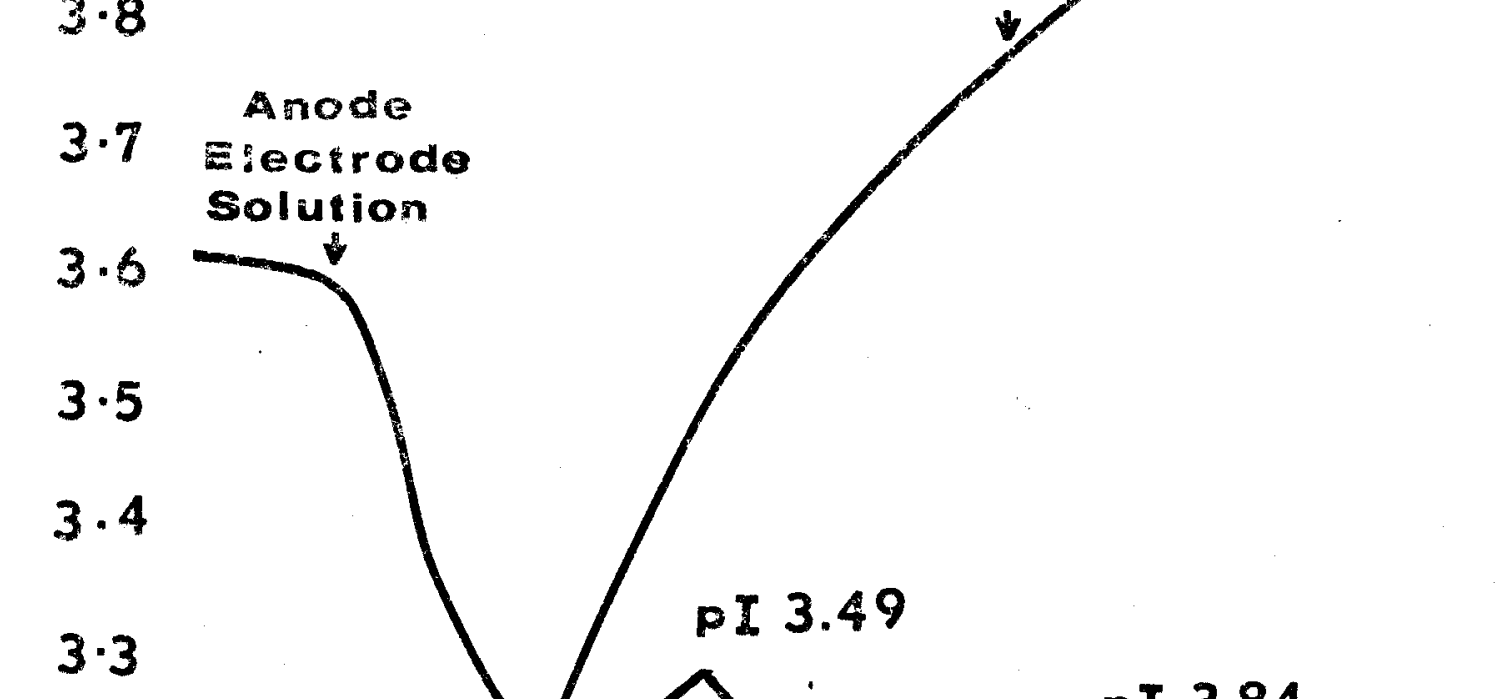

\section{$3 \cdot 3$}

$3 \cdot 2$

$3 \cdot 1$

$3 \cdot 0$

28

40

$5.260 \mathrm{ml}$

Figure 27. Separation of $14 \mathrm{mg}$ of Sephadex G-50 purified microsomal preparation by the LKB 8100 Electrofocusing Column in a pH gradient extending from pH 3 to 5. The curve with the two peaks shows the absorption of eluate at $280 \mathrm{~nm}$. The steadily increasing curve is a plot of the pH gradient superimposed. 
standard CT preparation been resolved in the same narrower $\mathrm{pH}$ gradient ( $\mathrm{pH} 3.0$ to 5.0 ) in which the microsomal CT preparations had been electrophoretically resolved. The isoelectric focusing procedure did establish the pI values of the protein components of both the standard and microsomal CT preparations, and the identity of the microsomal CT preparation with that of the standard CT preparation. It is also possible that the slight differences in pI values between the two resolved protein bands of the standard and microsomal CT preparations could be attributed to species differences in amino acid composition of the CT molecule. The standard porcine CT molecule is known to differ from ovine CT molecule at four positions, at amino acid residue number 14, 15, 19, and 22 (Figure 2), thereby resulting in slightly different pI values between the two molecules. Most of the amino acid differences between porcine and ovine CT are conservative. The phenylalanine residues at position 19 and 2 of the porcine molecule are replaced by tyrosine in the ovine CT molecule and the basic arginine residue at position 14 of the porcine molecule is replaced by lysine, another basic amino acid, in the ovine CT molecule. The only significant change in replacement of an amino acid residue is noted -at position 15 where the amino acid, amide asparagine, in the porcine molecule, is replaced by an aspartic acid residue in the ovine CT molecule. This change in amino acid at position 15 could account for the slight differences in the observed pI values.

Three forty-drop fractions corresponding to each of the two elution peaks in the resolved microsomal material were then passed through a column of Sephadex G-50 to remove most of the contamina- 
ting ampholytes from the resolved microsomal proteins. Approximately $2 \mathrm{mg}$ of purified microsomal material was obtained from each of the elution peaks. The radioactivity of $200 \mu \mathrm{g}$ of microsomal material from the elution peaks having pI values of 3.49 and 3.84 (Figure 27) was determined. It was not possible to determine the specific activity in terms of DPM/mg protein. There was still a slight amount of ampholyte contamination with each of the resolved microsomal preparation and it interfered with the determination of protein by the Lowry method. Therefore, radioactivity was expressed as counts per minute (CPM) per $200 \mu \mathrm{g}$ of sample. Neither resolved fraction exhibited enhanced incorporation of labeled glycine. The fraction having a pI of 3.49 had 238 CPM per $200 \mu \mathrm{gg}$ of sample, while that of the fraction having a pI of 3.84 had a value of 215 CPM per $200 \mu \mathrm{g}$ sample. Once again, this demonstrated that no preferential incorporation of glycine-C-14 into microsomal proteins was occurring.

The two electrophoretically resolved microsomal fractions were then assayed for hypocalcemic activity. In order to have sufficient quantities of resolved microsomal material for biological assay determinations, three isoelectric focusing runs were required. These were carried out as previously described. In the three instances, the microsomal material was electrophoretically resolved into two protein bands and the pI values of each band did not differ by more than $\pm 0.1 \mathrm{pH}$ unit. Bioassays were performed as previously described; however, in this instance, the injection dose was $1,000 \mu \mathrm{g}$ of electrophoretically resolved and Sephadex G-50 purified microsomal CT preparation. The hypocalcemic activity of 
these preparations are shown in Table XVIII. The microsomal CT material having a pI value of approximately 3.5 decreased serum calcium by $9.6 \%$ while the change in serum calcium was $0.031 \mathrm{mg} \%$ per $10 \mu \mathrm{g}$ protein. Injection of microsomal CT material having a pI value of approximately 3.8 resulted in a similar hypocalcemic response, since the serum calcium was decreased by $8.8 \%$ and the change in serum calcium was $0.027 \mathrm{mg} \%$ per $10 \mathrm{~kg}$ of protein.

The hypocalcemic responses of the two electrophoretically resolved microsomal CT fractions having pI values of 3.49 and 3.84 were compared to that of the unresolved microsomal CT preparation obtained from an incubation medium containing a physiological calcium concentration. The statistical evaluation of the hypocalcemic responses is shown in Table XIX. The resolved microsomal protein bands with isoelectric points of 3.49 and 3.84 were not statistically different in their hypocalcemic response from that of unresolved microsomal material. 
TABLE XVIII

HYPOCALCENIC ACTIVITY OF ELECTROPHORETICALLY RESOLVED MICROSOMAL* PREPARATIONS OBTAINED FROM AN INCUBATION MEDIUM CONTAINING

A PHYSIOLOGICAL CALCIUM CONCENTRATION

AND $2.5 \mu \mathrm{Ci}$ OF GLYCINE-C-14 (UL)

\begin{tabular}{|c|c|c|c|c|c|c|c|}
\hline Preparation & $\begin{array}{c}\text { Animal } \\
\text { No. }\end{array}$ & $\frac{\mathrm{Ca}}{\mathrm{t}_{0}}$ & $\frac{m g \%}{t_{50}}$ & $\begin{array}{r}\Delta \\
10\end{array}$ & $\begin{array}{l}\text { Ca mg\%/ } \\
\mu g \text { Protein }\end{array}$ & $\begin{array}{l}\text { Net } \\
\text { Change }\end{array}$ & $\begin{array}{l}\% \\
\text { Change }\end{array}$ \\
\hline Microsomal & 1 & 11.50 & 10.45 & & -0.031 & -1.05 & -9.1 \\
\hline \multirow[t]{5}{*}{$p I=3.49$} & 2 & 11.35 & 10.10 & & -0.037 & -1.25 & -11.0 \\
\hline & 3 & 10.51 & 9.94 & & $-0.01 ?$ & $-0.5 ?$ & -5.4 \\
\hline & 4 & 11.41 & 10.17 & & -0.037 & -1.24 & -10.9 \\
\hline & 5 & 10.52 & 9.34 & & -0.035 & -1.18 & -11.2 \\
\hline & \multicolumn{3}{|c|}{ Mean $\pm S . D$. } & & \multicolumn{2}{|c|}{$-0.031 \pm 0.010$} & -9.5 \\
\hline Microsomal & 1 & 10.10 & 9.25 & & -0.025 & -0.85 & -8.4 \\
\hline \multirow[t]{4}{*}{$p I=3.84$} & 2 & 10.93 & 10.40 & & -0.016 & -0.53 & -4.8 \\
\hline & 3 & 9.60 & 9.10 & & -0.015 & -0.50 & -5.2 \\
\hline & 4 & 10.06 & 8.99 & & -0.032 & -1.07 & -10.6 \\
\hline & 5 & 11.00 & 9.40 & & -0.047 & -1.60 & -14.5 \\
\hline
\end{tabular}

* Pourteen milligrams of Sephadex G-50 purified microsomal material Was electrophoretically fractionated into two protein peaks in an LKB 8100 Electrofocusing Column in a pH gradient extending from $\mathrm{pH} 3$ to 5. The first resolved protein band had a pI of 3.49 and the second had a pI of 3.84 . 
TABLE XIX

THE HYPOCALCEMIC RESPONSE OF ELECTROPHORETICALLY RESOLVED MICROSOMAL PREPARATIONS*

Preparation

$\triangle \mathrm{Ca} \mathrm{mg \% /}$

$10 \mu \mathrm{g}$ Protein
$\%$

Change
, Microsomal

Microsomal $\mathrm{pI}=3.49$

Microsomal $\mathrm{pI}=3.84$

$$
-0.024 \pm 0.017
$$$$
-0.031 \pm 0.010
$$

NS
$-9.5$

$-11.5$

$-8.7$

NS 


\section{CHAPTER IV}

\section{DISCUSSION}

Since the postulated existence of calcitonin by Copp et al. (1962) and its discovery and isolation from the thyroid gland by Hirsch et al. (1963), a large body of information has accumulated concerning the cell of origin, chemistry, and sites of action of this hypocalcemic hormone. However, full clarification of the factors involved in the mechanism of action, metabolism, duration of action, control of secretion, and physiological role of this hormone in the maintenance of mammalian mineral homeostasis have not been elucidated.

This study was concerned with further clarification of one of these areas of investigation, the control of calcitonin secretion; hence, its localization in thyroid glands and factors involved in its biosynthesis. More specifically, this study dealt with the subcellular localization of this hypocalcemic hormone in ovine thyroid glands, determining the influence of varying calcium ion concentration on hormone biosynthesis at the subcellular level, and establishing the identity of the hypocalcemic species with a reference standard calcitonin preparation by polyacrylamide gel electrophoresis and by isoelectric focusing. In addition, this investigation has provided for the first time, in so far as is known, information on the isoelectric point(s) of standard porcine and microsomal ovine calcitonin preparations. 
Calcitonin is secreted by the thyroid gland into the general circulation when a naturally occurring stimulus of hypercalcemia occurs. Its action is to markedly retard bone resorption as evidenced by the rapid decrease in blood calcium levels. When calcitonin is administered to rats, it produces a hypocalcemia which is manifested shortly after hormone administration and reaches a peak approximately one hour after the hormone injection (Hirsch et al., 1964: Kumar et al., 1965) and it is effective even in parathyroidectomized rats (Gudmundsson et al., 1966). Considerable evidence has been presented which indicates that CT inhibits the PTH-induced resorptive process both in vivo and in vitro. In vivo experiments of Milhaud and Moukhtar (1966), Johnston and Diess (1966), Klein et al. (1967) have consistently demonstrated that CT is effective in blocking PTH-induced bone resorption and may play a role in preventing bone matrix degradation as evidenced by the dramatically decreased excretion of hydroxyproline in intact rats (Martin et al., 1966, and Rasmussen et al., 1967). These experiments provided evidence for the concept that CT administration retarded all phases of bone resorption and that its action was not by direct inhibition of PTH.

The characteristic hypocalcemic response and the retardation of PTH-induced mineral resorption by $\mathrm{CT}$ has been demonstrated in in vitro experiments utilizing bone cultures (Friedman and Raisz, 1965; Friedman et al., 1968; Aliapoulios et al., 1966; Raisz and Nieman, 1967; Reynolds and Dingle, 1968; and Brand and Raisz, 1972), and isolated and perfused limbs (MacIntyre et al., 1967). These experiments have clearly demonstrated that the hormone is bio- 
logically active in in vitro systems and that its action in these systems may provide valuable information about its normal physiological role in mammals.

The problems associated with the control of CT secretion have been studied extensively. The mechanism(s) that controls the level of circulating hormone is akin to that controlling PPH. It is well-established that PTH secretion is controlled directly by blood calcium concentration, hypocalcemia enhancing secretion and hypercalcemia abolishing secretion. Calcitonin secretion has also been shown to be dependent upon blood calcium concentration. The level of this hormone in the peripheral circulation is in direct proportion to increases in calcium concentration above normal levels. The observations of Copp et al. (1962), Kumar et al. (1963), Care (1965), and Care et al. (1968) provided ample evidence that the extent of CT secretion from the thyroid is directly dependent upon the calcium concentration of the blood flowing through the thyroid gland, rising as the calcium level increases and falling as it decreases. The ' $\mathrm{C}$ ' cells of the thyroid release $\mathrm{CP}$ only in response to the actual calcium concentration of the blood flowing through the thyroid and the secretion rate is not dependent on the rate, degree, or direction of calcium flux in the blood. These observations correlated well with the evidence presented by Foster et al. (1964) and Matsuzawa and Kurosumi (1967) who demonstrated that hypercalcemic stimulation resulted in a gradual decrease of densely staining secretory granules of the ' $C$ ' cells. The highly sensitive radioimmunoassay technique of Deftos et al. (1968), Lee et al. (1969) and Cooper et al. (1971) confirmed that CT is being 
continuously secreted, even at physiological concentrations of blood calcium and that a rapid increase in CT secretion resulted when hypercalcemia was induced followed by a rapid decline in hormone when normocalcemia was restored.

The feasibility of utilizing in vitro biosynthetic media for studying the biosynthesis and release of hormones has been wellestablished. In vitro biosynthetic media containing either slices or homogenates of such endocrine tissues as the hypothalamus (Takabatake and Sachs, 1964), anterior pituitary (Adiga et al.. 1965), parathyroid glands (Hamilton and Cohn, 1969 and Hamilton et al., 1971), and thyroid glands (Sorgente, 1969 and Radde et al., 1970) have been utilized to monitor the biosynthesis and release of hormones. These endocrine tissues exhibited the physiological regulatory mechanism in in vitro biosynthetic media which is typical of the gland in vivo, thereby providing a valuable insight into the factors involved in the physiological regulation of hormone synthesis and release.

Pertinent to this investigation, Sorgente (1969) utilized an in vitro biosynthetic medium containing thyroid gland slices in an effort to establish the role of calcium and magnesium ion at the tissue level on the synthesis and release of CT. Sorgente's biosynthetic media contained ovine thyroid slices which were incubated ( $4 \mathrm{hr}, 37^{\circ} \mathrm{C}$ ) with glycine-C-14 in Krebs-Ringer bicarbonate buffer containing glucose $(320 \mathrm{mg} / 100 \mathrm{ml})$ and all the amino acids of calcitonin $(20 \mathrm{mg} / \mathrm{l})$. The calcitonin, which was isolated by salt precipitation from incubated thyroid slices, was assayed for hypocalcemic activity in rats. Sorgente's experiments indi- 
cated that a high calcium concentration ( $45.6 \mathrm{mg} \%$ ) in the biosynthetic medium stimulated both synthesis and release of CT since there was a $50 \%$ increase in the hypocalcemic response above physiological levels. However, varying the magnesium ion concentration was without effect in the CT regulatory mechanism. Later, Radde et al. (1970) presented evidence that a high calcium concentration in the biosynthetic medium was directly responsible for enhanced CT release into biosynthetic media.

In this investigation, Sorgente's in vitro biosynthetic medium was utilized for the incubation of ovine thyroid slices. Further, his experimental findings were confirmed in this study and provided a working model to allow for extrapolation to the subcellular level. Thus, in this study, three distinct subcellular fractions, the microsomes, the mitochondria, and the nuclei were obtained from incubated thyroid slices by differential centrifugation and were assayed for hypocalcemic activity in rats to determine the subcellular locus of CT. The influence of a varying calcium ion concentration on biosynthesis at the subcellular level was investigated by utilizing incubation media containing low (4.8 mg\%), physiological ( $11.5 \mathrm{mg} \%$ ), and high (26.0 mg\%) calcium concentrations. The incorporation of glycine-C-14 into these subcellular fractions was monitored in an attempt to determine whether the hypocalcemic fraction could be shown to reflect an enhanced incorporation of labeled amino acid, a further evidence of calcitonin biosynthesis. In addition, this approach would determine whether the alterations in the calcium ion concentration could effect the extent of incorporation of labeled amino acid into 
the hypocalcemic fraction.

Assaying subcellular fractions for hormonal activity is not an unprecedented investigative procedure since Brown and Ulvedal (1960) localized hormonal activity in a number of anterior pituitary fractions. Similarly, Hamilton and Cohn (1969) demonstrated that the $105,000 \times \mathrm{g}$ particulate fraction of parathyroid glands exhibited hypercalcemic activity which is characteristic of PTH. Moreover, Bauer and Tietelbaum (1966) and Cooper and Tashjian (1966) provided evidence that the $100,000 \times \mathrm{g}$ particulate fraction of pigs and rats exhibited hypocalcemic activity.

In the work reported here, the microsomal subcellular preparation was the only fraction which consistently exhibited hypocalcemic activity and a maximal hypocalcemic response occurred at a dose of 2,000 $\mathrm{mg}$. Microsomal preparations obtained from biosynthetic media containing physiological and high calcium concentrations were demonstrated to have significant differences in hypocalcemic activity when compared to mitochondria and nuclei from these biosynthetic media. These experiments with unpurified subcellular preparations clearly demonstrated that the microsomes were the subcellular locus of this hypocalcemic principle.

The microsomal CT preparation was not inactivated by heat, thereby indicating its thermostability. The ability of this preparation to withstand high temperature might be attributed to the protective influence of non-specific protein macromolecular material associated with these crude subcellular preparations. However, the microsomal preparation was readily inactivated by the proteolytic enzyme, pepsin. These characteristics of the 
unpurified hypocalcemic principle in the microsomal fraction are in accordance with the findings of Tashjian and Warnock (1967) in their studies with crude extracts of porcine CT.

The experiments of Hamilton and Cohn (1969) and Hamilton et al. (1971) demonstrated that a labeled amino acid which was a constituent of bovine PTH could be preferentially incorporated into a $100,000 \times \mathrm{g}$ particulate fraction which elicited a hypercalcemic response when injected into rats. Moreover, the extent of incorporation of the labeled amino acid into the hypercalcemic fraction was dependent upon the calcium concentration of the biosynthetic medium. Incorporation of the labeled amino acid into the hypercalcemic fraction was inversely proportional to the calcium concentration of the biosynthetic medium, being decreased at high calcium concentrations and increased at low calcium concentrations. These in vitro observations correlated well with the normal physiological response of PTH to hypocalcemia.

A distinct possibility therefore existed that a tracer amino acid, such as glycine-C-14 (a prominent constituent of ovine CT), could be incorporated preferentially into the hypocalcemic microsomal fraction and that varying the calcium concentration of the biosynthetic media might influence the extent of incorporation of the labeled amino acid. However, this investigation revealed that no such preferential incorporation occurred. Similarly, the extent of incorporation of the labeled amino acid into the various subcellular fractions was not influenced by the calcium concentration of the biosynthetic medium, since no consistent trend in glycine-C-14 incorporation occurred among subcellular fractions 
obtained from incubation media containing low, physiological, and high calcium concentrations. However, it was apparent that glycine-C-14 was being incorporated non-specifically into all of the proteins associated with the microsomal, mitochondrial, and nuclear preparations and not specifically into the hypocalcemic microsomal fraction.

Although no consistent patterns of incorporation of label into subcellular preparations could be discerned, it was readily observed from the closely corresponding radioactivity, protein and elution peaks, that each of these subcellular fractions was rather homogeneous in its protein composition. For, unlike Sorgente's observation of multiple radioactivity and protein peaks at the tissue level, only a single elution, protein, and radioactivity peak was observed for each of these subcellular preparations. This clearly indicated that a single subcellular fractionation step resulted in more purified protein preparations, especially the hypocalcemic microsomal preparation, than had the harsher multi-step isolation scheme utilized by Sorgente. This study confirmed the feasibility of isolating CT by a completely different procedure than the commonly employed extraction method from whole thyroid glands.

Although no consistent patterns in radioactive label incorporation were observed, the influence of calcium concentration at the subcellular level was very prominent. A high calcium concentration (26.0 $\mathrm{mg} \%$ ) in the biosynthetic media resulted in a highly significant increase in the hypocalcemic response of Sephadex G-100 purified microsomal preparations resulting in 
almost a 2-fold increase in hypocalcemic activity as compared to the hypocalcemic response of microsomes obtained from incubation media containing physiological and low calcium concentrations which decreased serum calcium by approximately $12 \%$. This substantiated the concept that the effect of calcium ion was a direct one at the subcellular level as well as at the whole tissue level.

No alteration in the hypocalcemic response was detected in microsomes obtained from an incubation medium containing a low calcium concentration when the response was compared to that of microsomes derived from incubation media containing physiological levels of calcium. This indicated, possibly, that a four hour period of incubation of thyroid slices at a low calcium concentration ( $4.8 \mathrm{mg} \%$ ) was not adequate time to alter the biosynthetic process of the ' $C$ ' cells or that a sufficient amount of CT was present in the ' $C$ ' cells which could not be depleted in four hours. A third possibility was that CT biosynthetic mechanism(s) was only sensitive to increasing calcium ion concentration in the ' $C$ ' cells and not to calcium levels below physiological levels. It was also likely that the ' $C$ ' cells of the thyroid continued normal CT synthesis from residual unlabeled amino acids in the cells during short term $(4 \mathrm{hr})$ calcium deprivation. Since CT biosynthesis is significantly enhanced in the microsomal preparations obtained from incubation media containing a high calcium concentration, it is possible that calcium ion may exert its effect on hormone biosynthesis in a number of ways. High calcium ion concentration could possibly enhance the activity of one or more of the enzymes involved in the synthesis of the calcitonin molecule. 
The CT standard could be resolved in both cationic and anionic polyacrylamide gels. It is possible that two differently charged species of the standard porcine CT molecule were present, perhaps due to the method of extracting this hormone preparation from whole porcine thyroid glands. A more satisfactory explanation is not presently available since the porcine CT extraction procedure, utilized by Dr. Robert Schleuter of Armour Pharmaceuticals, Kankakee, Illinois, is now being patented and is therefore not available for inspection.

The microsomal material could be electrophoretically fractionated only in anionic polyacrylamide gels. It is likely that the native ovine molecule was therefore predominantly negatively charged since migration of the microsomal material proceeded towards tha anode. A slight difference between the porcine and ovine CT molecule is that the ovine has an aspartate residue at position 15 whereas the porcine molecule has an asparagine residue and this may account for the ability of the ovine molecule to be resolved only in anionic gels.

The homogeneity of microsomal preparations was monitored after passage of the microsomal material through Sephadex G-100 and G-50 chromatographic columns. The Sephadex G-50 purification step had removed one of the protein bands seen in the Sephadex G-100 preparations. It was also apparent that a large portion of the hypocalcemic microsomal fraction was probably macromolecular material which was either uncharged or too large to be resolved in the small pore separation gel. Hence, the large quantities of sample required $(2,500 \mu \mathrm{g})$ to electrophoretically 
resolve the Sephadex G-100 and G-50 microsomal preparations as compared to the standard CT preparation $(333 \mu \mathrm{g})$. This does not present a serious disadvantage since it is likely that the isolation procedure for the standard porcine $\mathrm{CT}$ preparation required much larger quantities of porcine thyroid glands than did the subcellular fractionation procedure utilized in this study to obtain microsomal fractions.

The microsomal and standard CT preparations were further characterized by isoelectric focusing. This procedure allowed the first determination yet reported of the isoelectric points of these two molecules. The microsomal preparation was electrophoretically resolved into two protein bands having isoelectric points of 3.49 and 3.84 in a $\mathrm{pH}$ gradient extending from 3 to 5 . The standard porcine CT preparation was also resolved into two protein bands having quite similar isoelectric points of 3.70 and 3.94 in a $\mathrm{pH}$ gradient of 3 to 6 . The closeness of the isoelectric points of the electrophoretically resolved bands of the ovine and porcine CT preparations further established the identity of the hypocalcemic principle of the microsomal preparation with a standard CT preparation. The slight discrepancy in the pI values of the resolved band between the two CT preparations may be attributed to either the use of a broader $\mathrm{pH}$ gradient ( $\mathrm{pH} 3$ to 6) utilized for resolving the standard CT preparation or more likely, to the species differences already alluded to in amino acid composition of the CT molecule. This may result in a slightly different electrophoretic resolution of the porcine molecule in the electrofocusing column as compared to the ovine microsomal preparation. 
Each of the two electrophoretically resolved microsomal protein bands were found to contain hypocalcemic activity. It was therefore likely that the highly sensitive resolving power of the isoelectric focusing technique had separated two slightly different charged species of the same ovine CT molecule. The biological assay of electrophoretically resolved microsomal preparations was hampered by ampholyte contamination of the protein bands and therefore allowed for poor recovery of this purified material. This is attributable to the closeness of the molecular weights of the largest amprolyte species (M. W. to 1800 ) as compared to the CT molecule having a molecular weight of 3600 .

The fact that $C T$ was localized in the microsomal fraction, that its biosynthesis is enhanced by high calcium concentrations in in vitro biosynthetic media, and that the microsomal hormone preparation was characterized by polyacrylamide gel electrophoresis and isoelectric focusing, lays the groundwork for further studies. The subcellular fractionation technique may be utilized as an alternative large scale hormone isolation procedure for CT and possibly for other hormones such as insulin and glucagon of the pancreas, should they be associated with a particular subcellular fraction. It is an isolation and purification procedure which does not involve harsh extraction methods which tend to denature or alter the composition of biologically active species. The technique would be especially amenable to isolation of highly purified CT preparations from ultimobranchial tissues of fish or sharks which Copp and his associates have shown to have extremely 
potent CT preparations. This is possible since there is no contamination of the ${ }^{\prime} \mathrm{C}$ ' cells in this tissue with large amounts of thyroglobulin, thyroid hormones, and parathyroid tissue as occurs with the thyroid ' $C$ ' cells in most mammalian species. Moreover, the monitoring of labeled amino acid incorporation into a hypocalcemic subcellular preparation in an in vitro biosynthetic medium such as the one utilized in this study might be more advantageously studied in a system utilizing homogenates of ultimobranchial tissue. There would be two advantages to such a system since in a gland homogenate of ultimobranchial tissue, there would be fewer membranes present to hinder transport of a labeled amino acid and incorporation of label should proceed more rapidly. Secondly, one would not be hindered with the possibility of incorporation of tracer amino acid into as many non-specific proteins. 


\section{CHAPTER V}

\section{SUMMARY AND CONCLUSIONS}

From the information provided by the studies of Hamilton and Cohn (1969), Hamilton et al. (1971), and Sorgente (1969) on the in vitro biosynthesis of parathyroid hormone and calcitonin and the experiments of Brown and Ulvedal (1960) which localized hormonal activity of anterior pituitary within several subcellu. lar fractions, it appeared likely that the subcellular locus of ovine CT could be determined as well as the influence of calcium ion on hormone biosynthesis at the subcellular level. Consequently, ovine thyroid gland slices were incubated in in vitro biosynthetic media containing low ( $4.8 \mathrm{mg} \%$ ), physiological ( 11.5 $\mathrm{mg} \%$ ), and $\mathrm{righ}(26.0 \mathrm{mg} \%)$ calcium concentrations and $2.5 \mu \mathrm{Ci}$ of glycine-C-14, which served as a tracer amino acid. After incubation, the slices were homogenized and mitochondrial, nuclear, and microsomal subcellular fractions were obtained by differential centrifugation.

Microsomal, mitochondrial, and nuclear preparations were assayed for hypocalcemic activity at a dose of $2,000 \mu \mathrm{g}$. It was determined that the microsomes were the subcellular locus of calcitonin. A hypocalcemic microsomal preparation which had decreased serum calcium level of rats by $25 \%$ could not be inactivated by heating at $100^{\circ} \mathrm{C}$ for 2 hours in $0.01 \mathrm{~N} \mathrm{HCl}$. However, this same preparation was readily inactivated by incubation for 1 hour 
at $37^{\circ} \mathrm{C}$ with the proteolytic enzyme, pepsin, at a substrates enzyme weight ratio of 10 to 1 . These findings are known characteristics of unpurified calcitonin preparations.

Microsomal, mitochondrial, and nuclear preparations purified by passage through a column of Sephadex G-100 were assayed for hypocalcemic activity, protein content, and radioactivity. Once again, hypocalcemic activity was consistently demonstrated in the microsomal preparation. Microsomal preparations obtained from the low and physiological calcium concentration incubation media reduced serum calcium levels of rats by approximately $12 \%$. However, a microsomal preparation obtained from a high calcium concentration incubation medium decreased serum calcium levels of rats by $22.5 \%$, a highly significant increase in hypocalcemic activity. These findings indicated that a high calcium concentration in the in vitro biosynthetic media had stimulated calcitonin biosynthesis in the microsomes. The elution, protein, and radioactivity peaks of the mitochondrial, microsomal, and nuclear preparations corresponded closely. This indicated that the subcellular fractionation procedure could be effectively utilized as an alternative isolation procedure to the whole gland extraction methods for calcitonin. However, no preferential incorporation of glycine-C-14 into the hypocalcemic microsomal preparation was observed. The labeled amino acid was being incorporated nonspecifically into all three of these subcellular preparations.

Standard porcine and ovine microsomal calcitonin preparations were fractionated into their component protein bands by polyacrylamide gel electrophoresis. The microsomal fraction was inspected 
for homogeneity in polyacrylamide gels after passage through Sephadex G-100 and G-50 chromatographic columns. A comparison of the electrophoretic resolution of these preparations in anionic gels under identical conditions of milliamperage per gel, electrophoretic resolution time, and acrylamide gel concentration, established the identity of the hypocalcemic principle in the microsomal preparation with a standard porcine calcitonin preparation of known potency.

The isoelectric points of a microsomal and a standard calcitonin preparation of known potency ( 88 MRC Units per mg) were determined by isoelectric focusing. Fourteen milligrams of sephadex G-50 purified microsomal preparation were electrophoretically resolved in a pH gradient extending from $\mathrm{pH} 3$ to 5 into two protein bands having isoelectric points of 3.49 and 3.84 . Seven milligrams of the standard porcine calcitonin preparation were also resolved into two protein bands having quite similar isoelectric points of 3.70 and 3.94 in a pH gradient extending from $\mathrm{pH} 3$ to 6 . The highly sensitive resolving power of this technique was employed to obtain milligram quantities of each of the two electrophoretically resolved protein bands of the microsomal preparation. These two proteir fractions were both found to possess hypocalcemic activity when injected into rats.

This investigation indicated that the microsomal fraction is the locus of ovine calcitonin and that high calcium concentration enhances hormone biosynthesis of this fraction. In addition, the first determinations of the isoelectric points of standard porcine and microsomal ovine calcitonin preparations were established. 


\section{BIBLIOGRAPHY}

Adiga, P. R., I. Uemura, and T. Winnick. (1965) Biosynthesis of adrenocorticotropin and protein slices of bovine anterior pituitary tissue. Biochemistry 4, 246-252.

Aliapoulios, M., A. Savery, and P. Munson. (1965) New experiments with thyrocalcitonin. Fed. Proc. 24, 322.

Aliapoulios, M., P. Goldhaber, and P. Munson. (1966) Thyrocalcitonin inhibition of bone resorption induced by parathyroid hormone in tissue culture. Science 151, 330-331.

Aurbach, G. D. (1959) Isolation of parathyroid hormone after extraction with phenol. J. Biol. Chem. 234, 3179-3181.

Baghdiantz, A., G. V. Foster, A. Edwards, M. A. Kumar, E. Slack, H. A. Soliman, and I. MacIntyre. (1964) Extraction and purification of calcitonin. Nature (London) 203, 1027-1028.

Bauer, W. C. and S. L. Teitelbaum. (1966) Thyrocalcitonin activity of particulate fractions of the thyroid gland. Lab. Invest. 15, 323-329.

Belanger, I. (1965) Osteolysis: An outlook on its mechanism and causation. In "The Parathyroid Glands" (P. J. Gaillard, R. V. Talmage, and A. M. Budy, eds.) pp. 137-143, The University of Chicago Press, Chicago.

Belanger, I. F. and H. Rassmussen. (1968) Inhibition of osteocytic osteolysis by thyrocalcitonin and some anti-growth facfors. In "Parathyroid Hormone and Thyrocalcitonin (Calcitonin)" (R. V. Talmage and L. F. Belanger, eds.) pp. 156-168, Excerpta Medica Foundation, Amsterdam-C, The Netherlands.

Bell, P. H., W. R. Barg, Jr., D. F. Colucci, C. H. Davies, C. Dziobkowski, M. E. Englert, E. Heyder, R. Paul, and E. H. Snedeker. (1968) Purification and structure of porcine calcitonin-I. J.Am. Chem. Soc. 20, 2704-2706.

Bijvoet, 0., J. van der Sluys Veer, and A. Jansen. (1968) Effects of calcitonin on patients with Paget's disease, thyrotoxicosis, or hypercalcemia. Lancet $1_{2}$ 876-881.

Bijvoet, 0., J. van der Sluys Veer, H. de Vries, and A. Koppen. (197I) Natriuretic effect of calcitonin in man. N. Engl. J. Med. 284, 681-688. 
Brand, J。 and I.. Raisz. (1972) Effects of thyrocalcitonin and phosphate ion on the parathyroid stimulated resorption of bone. Endocrinology 90, 479-487.

Brewer, H. G., Jr., H. Keutmann, R. Reisfeld, P. Munson, R. Schleuter, and J.T. Potts, Jr. (1968) Role of sulfur-containing amino acids in the biological activity of porcine thyrocalcitonin (TC). Fed. Proc. 27, 690.

Brewer, H. B., Jr., H. Keutmann, J. T. Potts, Jr., R. A. Reisfeld, R. Schleuter, and P. L. Munson. (1968) Isolation and chemical properties of porcine thyrocalcitonin. J. Biol. Chem. 243, 57395747.

Brewer, H. B., Jr., R. Schleuter, and J. P. Aldred. (1970) Isolation and characterization of bovine thyrocalcitonin. J. Biol. Chem. 245, 4232-4240.

Brown, J. H. and F. Ulvedal. (1960) Studies on ultracentrifuge fractions of anterior pituitary with special reference to adrenocorticotrophic hormone. Endocrinology 66, 175-183.

Burr, R. G. (1970) An automated method for serum calcium utilizing ethylenedianinetetraacetic acid. C1in. Chem. 15, 1191-1197.

Bussolati, G. and A. G. E. Pearse. (1967) Immunofluorescent 10calization of calcitonin in the ' $\mathrm{C}$ ' cells of pig and dog thyroid. J. Endocrinol. 37, 205-210.

Care, A. D. (1965) Secretion of thyrocalcitonin. Nature (London) 205, 1289-1291.

Care, A. D., C. Cooper, T. Duncan, and H. Orimo. (1968) A study of thyrocalcitonin secretion by direct measurement of in vivo secretion rates in pigs. Endocrinology 83, 161-169.

Care, A. D., R. Bates, and H. Gitelman. (1971) Evidence for a role of cyclic AMP in the release of calcitonin. Ann. N. Y. Acad. Sc1. 185, 317-325.

Chase, L. and G. Aurbach. (1967) Parathyroid function and the renal excretion of $3^{\prime}, 5^{\prime}$-adenylic acid. Proc. Nat. Acad. Sci. 58, 518-525.

Chase, L. and G. Aurbach. (1968) Cyclic AMP and the mechanism of action of parathyroid hormone. In "Parathyroid Hormone and Thyrocalcitonin (Calcitonin)" (R.V. Talmage and L. F. Belanger 
eds.) pp. 247-257, Excerpta Medica Foundation, Amsterdam-C, The Netherlands.

Chase, L。, S. Fedak, and G. Aurbach. (1969) Activation of skeletal adenyl cyclase by parathyroid hormone in vitro. Endocrinology 84, 761-768.

Chase, L. and G. Aurbach. (1970) The effect of parathyroid hormone on the concentration of adenosine $3^{\prime}, 5^{\prime}$-monophosphate in skeletal tissue in vitro. J. Biol. Chem. 245, 1520-1526.

Chrambach, A., R. A. Reisfeld, M. Wyckoff, and J. Zaccari.

A procedure for rapid and sensitive staining of protein fractionated by polyacrylamide gel electrophoresis. Anal. Biochem. $\underline{20}, 150-154$.

Chrambach, A. and D. Rodbard. (1970) Polyacrylamide gel electrophoresis. Science 172, 440-451.

Cooper, C. W. and A. H. Tashjian, Jr. (1966) Subcellular localization of thyrocalcitonin. Endocrinology 79, 819-822.

Cooper, C., P. Hirsch, and P. Munson. (1970) Importance of endogenous thyrocalcitonin for protection against hypercalcemia in the rat. Endocrinology 86, 406-415.

Cooper, C., L. Deftos, and J. T. Potts, Jr. (1971) Direct measurement of in vivo secretion of pig thyrocalcitonin by radioimmunoassay. Endocrinology 88, 747-754.

Copp, D. H. and A. G. Davidson. (1961) Direct humoral control of parathyroid function in the dog. Proc. Soc. Exp. Biol. Med.107, $342-344$.

Copp, D. H., A. G. Davidson, and B. A. Cheney. (1961) Evidence for a new parathyroid hormone which lowers blood calcium. Proc. Can. Fed. Bio1. Sci. 4, 17.

Copp, D. H., E. C. Cameron, B. A. Cheney, A. G. Davidson, and K. G. Henze. (1962) Evidence for calcitonin -- a new hormone from the parathyroid that lowers blood calcium. Endocrinology 70, 638-649.

Copp, D. H. and K. G. Henze. (1964) Parathyroid origin of calcitonin. Evidence from perfusion of sheep glands. Endocrinology $75,49-55$. 
Copp, D. Ho, D. W. Cockcroft, and Y. Kueh. (1967a) Ultimobranchial origin of calcitonin. Hypocalcemic effect of extracts from chicken glands. Can. J. Physiol. Pharmaco1. 45, 1095-1099.

Copp, D. H., D.W. Cockcroft, and Y. Kueh. (1967b) Calcitonin from ultimobranchial glands of dogfish and chickens. Science $158,924-925$.

Copp, D. H. and C. O. Parkes。 (1968) Extraction of calcitonin from ultimobranchial tissue. In "Parathyroid Hormone and Thyrocalcitonin (Calcitonin)" (R. V. Talmage and L. F. Belanger, eds.) pp. 74-84, Excerpta Medica Foundation, Amsterdam-C, The Netherlands.

Deftos, L. J., M. R. Lee, and J. T. Potts, Jr. (1968) A radioimmunoassay for thyrocalcitonin. Proc. Nat. Acad. Sci. 60, 293-299.

Firschein, H., W. Neuman, G. Martin, and B. Mulryan. (1959) Studies on the mechanism of action of parathyroid hormone. Rec. Progr. Horm. Res. 15, 427-458.

Fleisch, H., G. Russel, and F. Straumann, (1966) Effect of pyrophosphate on hydroxyapatite and its implication in calcium homeostasis. Nature (London) 212, 901-903.

Foster, G. V., A. Baghdiantz, M. A. Kumar, E. Slack, H. A. Soliman, and I. MacIntyre. (1964) Thyroid origin of calcitonin. Nature (London) 202, 1303-1305.

Foster, G. V., I. MacIntyre, and A. G. Pearse. (1964) Calcitonin production and mitochondrion-rich cells of the dog thyroid. Nature (London) 203, 1029-1030.

Foster, G. V., G. Joplin, I. MacIntyre, K. Melvin, and E. Slack. (1966) Effect of thyrocalcitonin in man. Lancet $1,107-109$.

Foster, G. V., F。 Doyle, P. Bordier, and H. Matrajt. (1966) Effect of thyrocalcitonin on bone. Lancet $2,1428-1431$.

Foster, G. V., M. Clark, F. Doyle, G. Joplin, F. Singer, T. Fraser, and I. MacIntyre. (1968) Clinical applications of calcitonin. In "Parathyroid Hormone and Thyrocalcitonin (Calcitonin)" $\overline{(R}$. V. Talmage and L. F. Belanger, eds.) pp. 86-99, Excerpta Medica Foundation, Amsterdam-C, The Netherlands. 
Foster, G. V., P. Byfield, and T. Gudmundsson. Clinics Endocrinol. Metab. 1, 93-124.

Friedman, J. and L. G. Raisz. (1965) Thyrocalcitonin: inhibitor of bone resorption in tissue culture. Science 150, 1465-1467.

Friedman, J., W. Y. Au, and L. G. Raisz. (1968) Responses of fetal rat bone to thyrocalcitonin in tissue culture. Endocrinology $82,149-156$.

Fujita, R., H. Orimo, M. Ohata, M. Yoshikawa, K. Nakanishi, and E. Misaka. (1968) Prevention of experimental bone atrophy by thyrocalcitonin. Endocrinolo. Japon. 15, 8-12.

Gaillard, P. (1965) Observation on the effect of parathyroid products on explanted mouse limb-bone rudiments. In "The Parathyroid Glands" (P. J. Gaillard, R. V. Talmage, and A. M. Budy, eds.) pp. 146-169, The University of Chicago Press, Chicago.

Gittes, R., S. Toverud, and C. Cooper. (1968) Effects of hypercalcemia and hypocalcemia on the thyrocalcitonin content of rat thyroid glands. Endocrinology 82, 83-90.

Gley, E. (1891) Note sur les fonctions de la glande thyroide chez le lapin et chez le chien. C.R. H. Acad. Sci. 43, 843-847.

Goldhaber, P. (1965) Bone-resorption factors, cofactors, and giant vacuole osteoclasts in tissue culture. In "The Parathyroid Glands" (P. J. Gaillard, R. V. Talmage, and A. M. Budy, eds.) pp. 153-169, The University of Chicago Press, Chicago.

Gudmundsson, T. V., I. MacIntyre, and H. A. Soliman. (1966) The isolation of thyrocalcitonin and a study of its effects in the rat. Proc. Roy. Soc. London 164, 460-477.

Guttmann, S. T., J. Pless, E. Sandrin, P. A. Jaquenoud, H. Bossert, and H. Willems. (1968) Synthese des thyreocalcitonins. Helv. Chim. Acta. 51, 1155-1158.

Haas, H., and M. Dambacher. (1968) Thyrocalcitonin effects in man. Helv. Mied. Acta. $34,327-336$.

Habener, J. F., F. R. Singer, L. J. Deftos, R. M. Neer, and J. T. Potts, Ir. (1971) Explanation for unusual potency of salmon calcitonin. Nature (London) 232, 91-92. 
Hamilton, J. W. and D. V. Cohn. (1969) Studies on the biosynthesis in vitro of parathyroid hormone. I. Synthesis of parathyroid hormone by bovine parathyroid gland slices and its control by calcium. J.Bio1. Chem. 244, 5421-5429.

Hamilton, J. W., F. W. Spierto, R. R. MacGregor, and D. V. Cohn. (1971) Studies on the biosynthesis in vitro of parathyroid hormone. II. The effect of calcium and magnesium on synthesis of parathyroid tissue and incubation medium. J. Biol. Chem. $246,3224-3233$.

Harris, E. D., Jro, and A. Sjoerdsma. (1966) Effect of parathyroid extract on collagen metabolism. J. of Clin. Endocrinol. Metab. 26, 358-359。

Hirsch, P. F., and P. L. Munson. (1963) Hypocalcemic effect of thyroid extracts in rats. Pharmacologist $5,272$.

Hirsch, P. F., G. F. Gauthier, and P. L. Munson. (1963) Thyroid hypocalcemic principle and recurrent laryngeal nerve injury as factors affecting the response to parathyroidectomy in rats. Endocrinology 63, 244-252.

Hirsch, P. F., E. F. Voelkel, and P. L. Munson. (1964) Thyrocalcitonin: Hypocalcemic hypophosphatemic principle of the thyroid gland. Science 146, 412-413.

Johnston, C. C., Jr., and W. P. Deiss, Jr. (1966) An inhibitory effect of thyrocalcitonin on calcium release in vivo on bone metabolism in vitro. Endocrinology 78, $1139-\overline{114} \overline{3}$.

Kenny, A. D. (1964) Discussion. Rec. Progr. Horm. Res. 20, 84-85.

Keutmann, H. T., J. A. Parsons, J. T. Potts, Jr., and R. J. Schleuter. (1970) Isolation and chemical properties of two calcitonins from salmon ultimobranchial glands. J. Biol. Chem. 245, 1491-1496.

Klein, D. C., H. Morii, and R. V. Talmage. (1967) Effect of thyrocalcitonin administered during peritoneal lavage on removal of bone salts and their radioisotopes. Proc. Soc. Exp. Biol. Med. 124, 627-633.

Klein, D. and R. Talmage. (1968) Evidence for the secretion of thyrocalcitonin at normal and subnormal plasma calcium levels. Endocrinology 82, 132-136. 
Kumar, M. A., E. Slack, A. Edwards, H. A. Soliman, A. Baghdiantz, G. V. Foster, and I. MacIntyre. (1965) A biological assay for calcitonin. J. Endocrinol. 33, 469-475.

Larson, B., C. Owman, and F. Sundler. (1966) Monoaminergic mechanisms in parafollicular cells of the mouse thyroid gland. Endocrinology 78, 1109-1114.

Lee, M. R., L. J. Deftos, and J. T. Potts, Jr. (1969) Control of secretion of thyrocalcitonin in the rabbit as evaluated by radioimmunoassay. Endocrinology 84, 36-40.

Lowry, O. H., N. J. Rosebrough, A. L. Farr, and R. J. Randall. (1951) Protein measurement with the Folin phenol reagent. J. Biol. Chem. 193, 265-275.

MacCallum, W. G. and C. Voegtiin. (1909) On the relation of tetany to the parathyroid glands and to calcium metabolism. J. Exp. Med. 11, 118-151.

MacIntyre, I. (1970) Human calcitonin; Practical and theoretical consequences. In "Calcitonin 1969. Proceedings of the Second International symposium" (S. Taylor, ed.) pp. 56-73, Springer-Verlag Inc., New York.

MacIntyre, I., G. V. Foster, and M. A. Kumar. (1965) The thyroid origin of calcitonin. In "The Parathyroid Glands" (P. J. Gaillard, R. V. Talmage, and A. M. Budy, eds.), pp. 89-102. The University of Chicago Press, Chicago.

MacIntyre, I., J. A. Parsons, and C. J. Robinson. (1967) The effect of thyrocalcitonin on blood-bone calcium equilibrium in perfused tibia of the cat. J. Physiol. (London) 191, 393-406.

Mahler, H. R. and E. H. Cordes. (1966) Intracellular organization and particles. In "Biological Chemistry" (Harper and Row, Publishers) p. 394.

Martin, T. J., C. J. Robinson, and I. MacIntyre. (1966) The mode of action of thyrocalcitonin. Lancet I, 900-902.

Matsuzawa, T. and K. Kurosumi. (1967) Morphological changes in the parafollicular cells of the rat thyroid glands after administration of calcium shown by electron microscopy. Nature (London) 213, 927-928. 
McLean, F. C. (1957) The parathyroid hormone and bone. Clin. Orthodontics 9, 46-60.

McLean, F. C. and M. R. Urist. (1955) An introduction to the physiology of skeletal tissue. In "Bone" The University of Chicago Press, Chicago and London, Chapter X.

Milhaud, G. (1968) Utilization of thyrocalcitonin in man in normal and pathological conditions. In "Parathyroid Hormone and Thyrocalcitonin (Calcitonin)" (R. V. Talmage and L. F. Belanger, eds.), pp. 86-99. Excerpta Medica Foundation, Amsterdam-C, The Netherlands.

Milhaud, G. and M. S. Moukhtar. (1966) Thyrocalcitonin effects on calcium kinetics in the rat. Proc. Soc. Exp. Biol. Med. 123, 207-209.

Minkin, C. and R. Talmage. (1968) A study of the secretion and function of thyro-calcitonin in normal rats. In "Parathyroid Hormone and Thyrocalcitonin (Calcitonin)" (R. V. Talmage and L. F. Belanger, eds.) pp. 339-351. Excerpta Medica Foundation, Amsterdam-C, The Netherlands.

Morii, H. and H. Deluca. (1967) Relationship between vitamin D deficiency, thyrocalcitonin, and parathyroid hormone. Amer. J. Physiol. 213, 358-362.

Munson, P. L., P. F。 Hirsch, A. H. Tashjian, Jx., and M. A. Aliapoulios. (1966) Calcitonin and thyrocalcitonin; evaluation of hypocalcemic factors. In "Methods in Drug Evaluation" (P. Mantegazza and F. Piccinini, eds.) Pp. 467-480. Amsterdam, North-Holland.

Munson, P. L., J. T. Potts, Jr., R. A. Reisfeld, C. W. Cooper, and E. F. Voelkel. (1966) Further purification of pig thyrocalcitonin. Science 154, 425 .

Munson, P. L., P. F. Hirsch, H. B. Brewer, R. A. Reisfeld, C. W. Cooper, A. B. Wasthed, H. Orimo, and J. T. Potts, Jr. (1968) Thyrocalcitonin. Rec. Progr Horm. Res. 24, 589-650. 
Munson. P. I. and T. Gray. (1970) Function of thyrocalcitonin in normal physiology. Fed. Proc. 29, 1206-1208.

Murad, F., H. Brewer, and M. Vaughan. (1970) Effect of thyrocalcitonin on adenosine $3^{\prime}, 5^{\circ}$-cyclic phosphate formation by rat kidney and bone. Proc. Nat. Acad. Sci. 65, 446-453.

Neher, R., B. Riniker, H. Zuber, W. Rittel, and F. Kahnt, (1968) Thyrocalcitonin. II. Structur von $\alpha$-thyrocalcitonin. Helv. Chim. Acta. 21, 917-924.

Niall, H. D., H. T. Keutmann, D. H. Copp, and J. T. Potts, Jr. (1969) Amino acid sequence of salmon ultimobranchial calcitonin. Proc. Nat. Acad. Sci. 64, 771-778.

Niall, H. D., H. T. Keutmann, R. Sauer, M. Hogan, B. F. Dawson, G. D. Aurbach, and J. T. Potts, Jr. (1970) The amino acid sequence of bovine parathyroid hormone. I. Hoppe Seyler's Z. Physiol. Chem. 351, 1586-1588.

Nisbet, J. and B. Nordin. (1968) Thyrocalcitonin inhibition of bone resorption in tissue culture. In "Calcitonin: Proceedings of the Symposium on Thyrocalcitonin and the C Cells" (S. Taylor, ed.) pp. 230-237, Springer-Verlag Inc., New York.

Nonidez, J. F. (1931) The origin of the "parafollicular" cell, a second epithelial component of the thyroid gland of the dog. Amer. J. Anat. 49, 479-505.

0'Dor, P. K., C. O. Parkes, and D. H. Copp. (1969a) Amino acid composition of salmon calcitonin. Can. J. Biochem. 47, 832-825.

0'Dor, P. K., C. O. Parkes, and D. H. Copp. (1969b) Biological activities and molecular weights of ultimobranchial and thyroid calcitonins. J. Biochem. Physiol. 29, 295-304.

Orimo, H., M. Ohata, and T. Fujita. (1971) Role of inorganic pyrophosphatase in the mechanism of action of parathyroid hormone and calcitonin. Endocrinology 89, 852-858.

O'Riordan, J. L, , A. H. Tashjian, Jr., P. L. Munson, P. G. Condliffe, G. D. Aurbach. (1966) Thyrocalcitonin: ultracentrifugation in a gradient of sucrose. Science 154, 885-886.

O'Riordan, J. L. and G. D. Aurbach. (1968) Mode of action of thyrocalcitonin. Endocrinology 82, 377-383. 
Oyama, V. I. and H. Eagle. (1956) Measurement of cell growth in tissue culture with phenol reagent. Proc. Soc. Exp. Biol. Med. 91, 305-307.

Park, H. and R. Talmage. (1968) Comparison of the effects of calcium and endogenous parathyroid hormone on RNA synthesis in rat bone. In "Parathyroid Hormone and Thyrocalcitonin (Calcitonin)" (R. V. Talmage and L. F. Belanger, eds.) pp. 203214, Excerpta Medica Foundation, Amsterdam-C, The Netherlands.

Pearse, A. G. (1966) The cytochemistry of the thyroid cells and their relationship to calcitonin. Proc. Roy. Soc., London 164, 478-487.

Pearse, A. G. and A. F. Carvalheira. (1967) Cytochemical evidence for an ultimobranchial origin of rodent thyroid $C$ cells. Nature (London) 214, 929-930.

Potts, J. T., Jr., R. A. Reisfeld, P.F. Hirsch, A. B. Wasthed, E. F. Voelkel, and P. L. Munson. (1967) Purification of porcine thyrocalcitonin. Proc. Nat. Acad. Sci. 58, 328-335.

Potts, J. T., Jr., H. D. Niall, H. T. Keutmann, H. B. Brewer, Jr., and L. J. Deftos. (1968) The amino acid sequence of porcine thyrocalcitonin. Proc. Nat. Acad. Sci. 59, 1321-1328.

Potts, J. T., Jr., H. T. Keutmann, H. D. Niall, L. J. Deftos, H. B. Brewer, and G. D. Aurbach. (1968) Covalent structure of bovine parathyroid hormone in relation to biological and immunological activity. In "Parathyroid Hormone and Thyrocalcitonin (Calcitonin)" (R.V. Talmage and L。F. Belanger, eds.) pp. 44-53. Excerpta Medica Foundation, Amsterdam-C, the Netherlands.

Potts, J. T., Jr., H. D. Niall, H. T. Keutmann, L. J. Deftos, and J. A. Parsons. (1970) Calcitonin: Recent chemical and immunological studies. In "Calcitonin 1969. Proceedings of the Second International Symposium" (S. Taylor, ed.) pp. 56-73. Springer-Verlag Inc., New York.

Potts, J. T., Jr., G. W. Tregear, H. T. Keutmann, H. D. Niall, R。 Sauer, L. J. Deftos, B. F. Dawson, M. L. Hogan, and G. D. Aurbach。 (1971) Synthesis of a biologically active $N$-terminal tetratriacontapeptide of parathyroid hormone. Proc. Nat. Acad. Sci. 68, 63-67. 
Potts, J. T., Jr., H. T. Keutmann, H. D。 Niall, and G. W. Tregear. (1971) The chemistry of parathyroid hormone and the calcitonins. Vitamins and Hormones 29, 41-93.

Putter, I., E. A. Kaczka, R. E. Harman, E. L. Rickes, A. J. Kempf, L. Chaiet, J. W. Rothrock, A. W. Wase, and F. J. Wolf. (1967) The isolation and properties of thyrocalcitonin. J. Amer. Chem. Soc. $89,5301-5302$.

Radde, I., D. Parkinson, E. Witterman, and B. Hoffken. (1970) Magnesium and calcium stimuli to in vitro release of calcitonin from thyroid slices. In "Calcitonin 1969. Proceedings of the Second International Symposium" (S.Taylor, ed.) pp. 376-380, Springer-Verlag Inc., New York.

Raisz, L. G. and I. Niemann. (1967) Early effects of parathyroid hormone and thyrocalcitonin on bone in tissue culture. Nature (London) 214, 486-487.

Rasmussen, H. and L. C. Craig. (1959) Purification of parathyroid hormone by counter-current distribution. J.Am. Chem. Soc. 81, 5003 .

Rasmussen, H. and A. Tenenhouse. (1967) Thyrocalcitonin, osteoporosis, and osteolysis. Amer. J. Med. 43, 711-726.

Rasmussen, H., C. Anast, and C. Arnaud. (1967) Thyrocalcitonin, EGTA, and urinary electrolyte excretion. J. Clin. Invest. 46, 746-752。

Reynolds, J. J. (1968) Inhibition by calcitonin of bone resorption induced in vitro by vitamin A. Proc. Roy. Soc., London 170, 61-69.

Reynolds, J. J. and J. T. Dingle. (1968) Time course of action of calcitonin on resorbing mouse bones in vitro. Nature (London) 218, 1178-1179.

Reynolds, J. J., J. T. Dingle, and T. V. Gudmundsson. (1968) Bone resorption in vitro and its inhibition by calcitonin. In "Calcitonin: Proceedings of the Symposium on Thyrocalcitonin and the C Cells" (S. Taylor, ed.) pp. 223-229, Springer-Verlag Inc., New York.

Riniker, B., R. Neher, R. Maier, F。 Kahnt, P. Byfield, T. Gudmundsson, L. Galante, and I. MacIntyre. (1968) Menschlisches calcitonin I. Isolierung und charakterisierung. He1v. Chim. Acta。 51, 924-928. 
Rittel, W., M. Brugger, B. Kamber, B. Riniker, and P. Sieber. (1968) Thyrocalcitonin III. Die synthese des $\alpha$-thyrocalcitonins. Helv. Chim. Acta. 51, 924-928.

Ritzen, M., L. Hammarstrom, and L. Ullberg. (1965) Autoradiographic distribution of 5-hydroxytryptamine and 5-hydroxytryptophan in the mouse. Biochem. Pharmacol. 14, 313-321.

Robinson, C., T. Martin, E. Mathews, and I. MacIntyre. (1967) Mode of action of thyrocalcitonin. J. Endocrinol. 32, 71-79.

Robinson, C., E. Mathews, and I. MacIntyre. (1969) The effect of parathyroid hormone and thyrocalcitonin on the intestinal absorption of calcium and magnesium. In "Calcified Tissues, Proceedings 5th European Symposium" pp. 279-282, Bordeaux, Paris.

Sanderson, P. H., F. Marshall, and R. E. Wilson. (1960) Calcium and phosphorus homeostasis in the parathyroidectomized dog: Evaluation by means of the EDTA and calcium tolerance tests. J. Clin. Invest. $39,662-670$.

Sandstrom, I. V. (1880) Glandulae parathyroideae. Uppsala Lak. For. Forh. 15, 441.-471.

Sauer, R., H. D. Niall, and J. T. Potts, Jr. (19?0) Accelerated procedures for automated peptide degradation. Fed. Proc. 29, 728.

Sieber, P., M. Brugger, B. Kamber, B. Riniker, W. Rittel, R. Maier, and M. Staeheling. (1970) Synthesis and biological activity of peptide sequences related to porcine $\alpha$-thyrocalcitonin. In "Calcitonin 1969. Proceedings of the Second International Symposium" (S. Taylor, ed.) pp. 28-33. Springer-Verlag Inc., New York.

Sorgente, N. (1969) The in vitro biosynthesis of calcitonin. Doctoral Dissertation, Loyola University of Chicago.

Stux, M., B. Thompson, H. Isler, and C. Leblond. (1961) The "light cells" of the thyroid gland of the rat. Endocrinology 68, 292-308.

Svensson, H. (1961) Isoelectric fractionation, analysis, and characterization of ampholytes in natural $\mathrm{pH}$ gradients. I. The differential equation of solute concentrations at a steady state and its solution for simple cases. Acta Chem. Scand. 15, $325-341$. 
Svensson, H. (1962) Isoelectric fractionation, analysis, and characterization of ampholytes in natural pH gradients. III. Description of apparatus for electrolysis in columns stabilized by density gradients and direct determination of isoelectric points. Arch. Biochem. Biophys. 1, 132-138.

Takabatake, Y. and H. Sachs. (1964) Vasopressin biosynthesis. III. In vitro studies. Endocrinology 25, 945-942.

Talmage, R. V. (1.967) A study of the effect of parathyroid hormones on bone remodeling and on calcium homeostasis. Clin. Orthop. Related Res. 54, 163-173.

Talmage, R. V., J. Neuenschwander, and L. Kraintz. (1964) Prescence of thyrocalcitonin in rats. Fed. Proc. 23, 204.

Talmage, R. V., J. Neuenschwander, and L. Kraintz. (1965) Evidence for the existence of thyrocalcitonin in the rat. Endocrinology 26, 103-10?.

Tashjian, A. H., Jr. (1965) Homeostasis of plasma calcium: Effects of actinomycin $D$, parathyroidectomy and thyrocalcitonin. Endocrinology $27,375-381$.

Tashjian, A, H., Jr. and D. R. Warnock. (1967) Stability of the hypocalcemic activity of porcine thyrocalcitonin. Endocrinolosy 81, $306-318$.

Tenenhouse, A., C. Arnaud, and H. Rasmussen. (1965) The isolation and characterization of thyrocalcitonin. Proc. Nat. Acad. Sci. 53, 818-822.

Tenenhouse, A. and H. Rasmussen. (1968) Parathyroid hormone, thyrocalcitonin and ascites tumor cells. In "Parathyroid Hormone and thyrocalcitonin (Calcitonin)" ( $R$. V. Talmage and L. F. Belanger, eds.) pp. 392-400. Excerpta Medica Foundation, Amsterdam-C, The Netherlands.

Vesterberg, 0. (1969) Synthesis and isoelectric fractionation of carrier ampholytes. Acta. Chem. Scand. 23, 2653-2666.

Vesterberg, 0. and H. Svensson. (1966) Isoelectric fractionation, analysis, and characterization of ampholytes in natural pH gradients. IV. Further studies on the resolving power in connection with separation of myoglobins. Acta. Chem. Scand. 20, 820-834. 
Wells, H. and W. Lloyd. (1967) Effect of theophylline on the serum calcium of rats after parathyroidectomy and administration of parathyroid hormone. Endocrinology 81, 139-144.

Wells, H. and W. Lloyd. (1968) Inhibition of the hypocalcemic action of thyrocalcitonin by theophyline and isoproterenol. Endocrinology 82, 468-474.

Wells, H. and W. Lloyd. (1968) Hypocalcemic effect of imidazole in rats. Endocrinology 83, 521-529.

Wener, J., S. Gorton, and L. Raisz. (1972) Escape from inhibition of resorption in cultures of fetal bone treated with calcitonin and parathyroid hormone. Endocrinology 90, 752-759.

Williams, D. E. and R. A. Reisfeld. (1964/65) Disc electrophoresis in polyacrylamide gels. Extension to new conditions of $\mathrm{pH}$ and buffer. Ann. N. Y. Acad. Sci. 121, 373-381. 


\section{ABSTRACT}

Abstract of the dissertation entitled "CALCITONIN ACTIVITY OF PARTICULATE FRACTIONS OF THE OVINE THYROID GLAND" submitted by Peter Rudolf oeltgen in partial fulfillment of the requirements for the degree of Doctor of Philosophy, February, 1973.

Ovine thyroid gland slices were incubated $\left(4 \mathrm{hr}, 37^{\circ} \mathrm{C}\right)$ in an in vitro biosynthetic medium consisting of a Krebs-Ringer bicarbonate buffer $(\mathrm{pH} 7.4)$, glucose $(320 \mathrm{mg} / 100 \mathrm{ml})$, all the amino acids of ovine calcitonin $(20 \mathrm{mg} / 1)$ and glycine-c-14. The incubations were carried out in media containing low $(4.8 \mathrm{mg} \%)$, physiological ( $11.5 \mathrm{mg} \%)$, and high $(26.0 \mathrm{mg} \%$ ) calcium concentrations. After incubation, the slices were homogenized and mitochondrial, nuclear, and microsomal subcellular fractions were obtained by differential centrifugation. After purification by passage through a column of Sephadex G-100, these fractions were assayed for hypocalcemic activity, protein content, and radioactivity.

Hypocalcemic activity was consistently demonstrated in the microsomal preparation. The microsomal fraction was found not to be inactivated by heat, but was readily inactivated by incubation with the proteolytic enzyme, pepsin, thereby exhibiting known characteristics of the molecule. Purified microsomal preparations derived from the low and physiological calcium concentration incubation media reduced serum calcium levels of rats by approximately 12\%. However, a microsomal preparation obtained from a high calcium concentration incubation medium decreased serum calcium levels of rats by $22.5 \%$, a highly significant increase. The elution, 
protein, and radioactivity peaks corresponded closely.

Standard and microsomal calcitonin preparations were fractionated into their protein components by polyacrylamide gel electrophoresis. The microsomal fraction was inspected for homogeneity in polyacrylamide gels after passage through Sephadex G-100 and G-50 chromatographic columns. A comparison of the electrophoretic resolution established the identity of the hypocalcemic principle in the microsomal preparation with a standard calcitonin preparation of known potency.

Isoelectric points of the microsomal and standard calcitonin preparations were determined by isoelectric focusing. The microsomal preparation was electrophoretically resolved in a pH gradient extending from 3 to 5 into two protein bands having isoelectric points of 3.5 and 3:8. The standard calcitonin preparation was also resolved into two protein bands having quite similar isoelectric points of 3.7 and 3.9 in a $\mathrm{pH}$ gradient of 3 to 6.

The results of this investigation indicated that the microsomal preparation was the only particulate fraction of ovine thyroid tissue that exhibited calcitonin activity and that high calcium concentration enhanced hormone biosynthesis in this fraction. Determination of the isoelectric points of ovine and porcine calcitonin was achieved by isoelectric focusing. 
APPROVAL SHEET

The dissertation submitted by Peter R. Oeltgen has been read and approved by a committee from the faculty of the Graduate School.

The final copies have been examined by the director of the dissertation and the signature which appears below verifies the fact that any necessary changes have been incorporated, and that the dissertation is now given final approval with reference to content, form and mechanical accuracy.

The dissertation is therefore accepted in partial fulfillmint of the requirements for the Degree of Doctor of Philosophy.

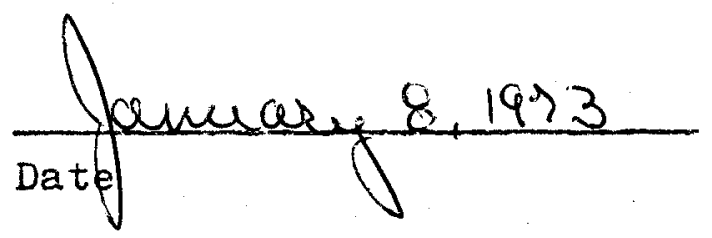
Maurice J. Pitteureuy 